\title{
Picard-Lefschetz Theory for the Universal Coverings of Complements to Affine Hypersurfaces
}

By

\author{
Ichiro SHIMADA *
}

\begin{abstract}
We study the global monodromy on the middle homology group of the universal coverings of the complements to non-singular affine hypersurfaces which intersect the hyperplane at infinity transversely. This monodromy can be regarded as a deformation of the monodromy on the middle homology group of the affine hypersurfaces. We show that this representation becomes irreducible when the deformation parameter is generic.
\end{abstract}

\section{\$0. Introduction}

Let $\Gamma$ denote the vector space $\Gamma\left(\mathbf{P}^{n}, \mathcal{O}(d)\right)$, and $\Gamma^{\times}$the space $\Gamma \backslash\{0\}$. We assume that $n \geq 2$ and $d \geq 3$. Let $\mathbf{P}_{*}(\Gamma)$ stand for the projective space $\Gamma^{\times} / \mathbb{C}^{\times}$, and pr: $\Gamma^{\times} \rightarrow \mathbb{P}_{*}(\Gamma)$ the natural projection. This space $\mathbf{P}_{*}(\Gamma)$ parameterizes all projective hypersurfaces of degree $d$ in $\mathbf{P}^{n}$. We fix a hyperplane at infinity $H_{\infty}$ in $\mathbf{P}^{n}$, and consider the affine space $\mathbf{A}^{n}:=\mathbf{P}^{n} \backslash H_{\infty}$. We define $U \subset \mathbf{P}_{*}(\Gamma)$ to be the locus of all projective hypersurfaces of degree $d$ which are non-singular and intersect $H_{\infty}$ transversely, and define $\mathcal{U}$ to be the pull-back of $U$ by the projec. tion :

$$
\mathcal{U}:=\operatorname{pr}^{-1}(U) \subset \Gamma^{\times}
$$

For $u \in \Gamma^{\times}$, let $f_{u}$ denote the corresponding homogeneous polynomial of degree d. We put

$$
\bar{X}_{u}:=\left\{f_{u}=0\right\}, Y_{u}:=\bar{X}_{u} \cap H_{\infty}, X_{u}:=\bar{X}_{u} \backslash Y_{u}, \text { and } E_{u}:=\mathbf{A}^{n} \backslash X_{u} .
$$

Communicated by K. Saito, December 11, 1995.

1991 Mathematics Subject Classification (s) : 14D05, 32S40

* Department of Mathematics, Faculty of Science, Hokkaido University, Sapporo 060, Japan. 
Then we have the monodromy representation

$$
\rho: \pi_{1}(U, \bar{b}) \rightarrow \operatorname{Aut}_{\mathbb{Z}}\left(H_{n-1}\left(X_{b} ; \mathbb{Z}\right)\right),
$$

where $b \in \mathcal{U}$ is a base point and $\bar{b} \in U$ is the point $\operatorname{pr}(b)$. This representation has been well investigated by the classical Picard-Lefschetz theory.

The purpose of this paper is to construct a certain kind of deformation of this classical monodromy representation.

The idea is to consider the middle homology group $H_{n}\left(F_{b} ; \mathbb{Z}\right)$ of the universal covering

$$
F_{b} \rightarrow E_{b}
$$

of the complement $E_{b}$. We cannot, however, define the acion of $\pi_{1}(U, \bar{b})$ on $H_{n}\left(F_{b} ; \mathbb{Z}\right)$ in a naive way, because the universal coverings $F_{u} \rightarrow E_{u}$ cannot be constructed universally over $U$. In order to construct the universal family of $F_{u}$, we will enlarge the base space $U$ to $U=\operatorname{pr}^{-1}(U)$. We fix an element $h \in$ $\Gamma\left(\mathbb{P}^{n}, \mathcal{O}(1)\right)$ which defines the hyperplane $H_{\infty}$. Suppose that $u \in \mathcal{U}$. When the defining equation $f_{u}$ of $\bar{X}_{u}$ is specified, the universal covering $F_{u} \rightarrow E_{u}$ of $E_{u}$ can be defined as the pull-back of the exponential map $\mathbb{C} \rightarrow \mathbb{C}^{\times}$by the polynomial map $f_{u} / h^{d}: E_{u} \rightarrow \mathbb{C}^{\times}$. Thus we can construct the universal family of the universal coverings over $U$. We will show that $\pi_{1}(U, b)$ is a central extension of $\pi_{1}(U, b)$ by $\mathbb{Z}$ (see Corollary 1.1).

Since Gal $\left(F_{u} / E_{u}\right) \cong \pi_{1}\left(E_{u}\right)$ is an infinite cyclic group, we can consider $H_{n}\left(F_{u} ; \mathbb{Z}\right)$ as a module over the ring of Laurent polynomials $\mathbb{Z}\left[q, q^{-1}\right]$, where the multiplication by $q$ is identified with the action of a generator of $\mathrm{Gal}\left(F_{u} / E_{u}\right)$ $\cong \mathbb{Z}$ on $H_{n}\left(F_{u} ; \mathbb{Z}\right)$. This action is also defined globally over $\mathcal{U}$.

Therefore, we get a monodromy representation

$$
\widetilde{\rho}: \pi_{1}(\mathcal{U}, b) \rightarrow \operatorname{Aut}_{\mathbb{Z}\left[q, q^{-1}\right]}\left(H_{n}\left(F_{b} ; \mathbb{Z}\right)\right)
$$

of $\pi_{1}(U, b)$ on the $\mathbb{Z}\left[q, q^{-1}\right]$-module $H_{n}\left(F_{b} ; \mathbb{Z}\right)$.

It is known that the complement $E_{b}$ is homotopically equivalent to the bouquet of $S^{1}$ and $N:=(d-1)^{n}$ copies of $S^{n}\left(\left[11 ;\right.\right.$ Corollary 1.2]). Hence $H_{n}\left(F_{b} ; \mathbb{Z}\right)$ is a free module of rank $N$ over $\mathbb{Z}\left[q, q^{-1}\right]$. This rank $N$ is equal with the $(n-1)$ -st Betti number of $X_{b}$. More specifically, we shall show that there exists an isomorphism 


$$
H_{n}\left(F_{b} ; \mathbb{Z}\right) \cong H_{n-1}\left(X_{b} ; \mathbb{Z}\right) \otimes_{\mathbb{Z}} \mathbb{Z}\left[q, q^{-1}\right]
$$

of $\mathbb{Z}\left[q, q^{-1}\right]$-modules such that the homomorphism $H_{n}\left(F_{b} ; \mathbb{Z}\right) \rightarrow H_{n-1}\left(X_{b} ; \mathbb{Z}\right)$ obtained from $(0.3)$ combined with the homomorphism $H_{n-1}\left(X_{b} ; \mathbb{Z}\right) \otimes \mathbb{Z}\left[q, q^{-1}\right]$ $\rightarrow H_{n-1}\left(X_{b} ; \mathbb{Z}\right)$ given by $q \mapsto 1$ is $\pi_{1}(\mathcal{U}, b)$-equivariant (see Theorems 6.1 and Remark 7.1). Here $\pi_{1}(\mathcal{U}, b)$ acts on $H_{n}\left(F_{b} ; \mathbb{Z}\right)$ by $\widetilde{\rho}$, and on $H_{n-1}\left(X_{b} ; \mathbb{Z}\right)$ by $\rho$ composed with the natural surjective homomorphism $\pi_{1}(\mathcal{U}, b) \rightarrow \pi_{1}(U, \bar{b})$ induced by the projection $\mathrm{pr}: \mathcal{U} \rightarrow U$.

This isomorphism (0.3) enables us to regard $\widetilde{\rho}$ as a deformation of the classical monodromy $\rho$ in (0.1). Suppose that we are given a non-zero complex number $\alpha$. We can consider $\mathbb{C}$ as a $\mathbb{Z}\left[q, q^{-1}\right]$-module by identifying $q$ with $\alpha$. Then the isomorphism (0.3) implies the isomorphism between complex vector spaces

$$
H_{n}\left(F_{b} ; \mathbb{Z}\right) \otimes_{\mathbb{Z}\left[q, q^{-1}\right]} \mathbb{C} \cong H_{n-1}\left(X_{b} ; \mathbb{Z}\right) \otimes_{\mathbb{Z}} \mathbb{C} \cong H_{n-1}\left(X_{b} ; \mathbb{C}\right)
$$

Evaluating $\widetilde{\rho}$ at $q=\alpha$ and using this isomorphism, we obtain a representation

$$
\rho(\alpha): \pi_{1}(U, b) \rightarrow \operatorname{Aut} \mathbf{C}\left(H_{n-1}\left(X_{b} ; \mathbb{C}\right)\right),
$$

and thus we get a family of representations $\{\rho(\alpha)\}$ parameterized by all non-zero complex numbers. The property of the isomorphism (0.3) implies that $\rho(1)$ is nothing but the complexified classical representation $\rho \otimes_{\mathbf{Z}} \mathbb{C}$ composed with the homomorphism $\pi_{1}(U) \rightarrow \pi_{1}(U)$.

The main theorem of this paper is as follows. Let $\mathbf{Q}(q)$ denote the quotient field of $\mathbb{Z}\left[q, q^{-1}\right]$.

Irreducibility Theorem. The monodromy representation of $\pi_{1}(\mathcal{U}, b)$ on the vector space $H_{n}\left(F_{b} ; \mathbb{Z}\right) \otimes_{\mathbf{Z}\left[q, q^{-1}\right]} \mathbb{Q}(q)$ induced from $\widetilde{\rho}$ is absolutely irreducible.

Corollary. If $\alpha$ is general enough, then $\rho(\alpha)$ is irreducible.

This shows that our deformation is non-trivial, because the classical representation $\rho \otimes_{\mathbf{Z}} \mathbf{C}$ is not irreducible. In fact, $\rho \otimes_{\mathbf{Z}} \mathbf{Q}$ is composed of the following two representations on the primitive parts of middle cohomology groups :

$$
\begin{array}{ll}
\rho_{0} & : \pi_{1}(U, \vec{b}) \rightarrow \operatorname{Aut}_{\mathbf{Q}}\left(H_{\text {prim }}^{n-1}\left(\bar{X}_{b} ; \mathbf{Q}\right)\right), \text { and } \\
\rho_{\infty}: \pi_{1}(U, \bar{b}) \rightarrow \operatorname{Aut}_{\mathbf{Q}}\left(H_{\text {prim }}^{n-2}\left(Y_{b} ; \mathbf{Q}\right)\right) ;
\end{array}
$$


that is, there exists an exact sequence

$$
0 \rightarrow H_{\text {prim }}^{n-2}\left(Y_{b} ; \mathbb{Q}\right) \rightarrow H_{n-1}\left(X_{b} ; \mathbb{Q}\right) \rightarrow H_{\text {prim }}^{n-1}\left(\bar{X}_{b} ; \mathbb{Q}\right) \rightarrow 0
$$

which is preserved by the monodromy action of $\pi_{1}(U, \bar{b})$. This exact sequence follows from the isomorphism $H_{n-1}\left(X_{b} ; \mathbb{Q}\right) \cong H^{n-1}\left(\bar{X}_{b}, Y_{b} ; \mathbb{Q}\right)$. It corresponds to the weight filtration of the mixed Hodge structure on the middle term $([2])$, and hence is preserved by the monodromy action. The old Picard-Lefschetz theory tells us the following:

\section{Theorem. Both of $\rho_{0}$ and $\rho_{\infty}$ are absolutely irreducible.}

Therefore, our deformation fuses these two irreducible representations into one big irreducible representation.

Our irreducibility theorem can be regarded as a generalization of the well-known fact that the reduced Burau representation of Artin's braid group is irreducible (cf. [1]). To see this, let us consider the case when $n=1$. In this case, $\pi_{1}(U)$ is Artin's braid group $B_{d}$ of $d$ strings. We fix an affine coordinate $t$ of $\mathbb{P}^{1}$ such that $H_{\infty}=\{t=\infty\}$. Then we have a normalized defining equation

$$
f_{u}^{*}:=\left(t-a_{1}\right)\left(t-a_{2}\right) \ldots\left(t-a_{d}\right) \in \mathcal{U}
$$

of $\bar{X}_{u} \in U$ characterized by the property that the coefficient of $t^{d}$ is 1 . This means that there exists a section $s$ of the projection $U \rightarrow U$ given by $f_{s(\operatorname{pr}(u))}=$ $f_{u}^{*}$. Hence $\pi_{1}(U)$ is isomorphic to the product $\mathbb{Z} \times \pi_{1}(U) \cong \mathbb{Z} \times B_{d}$. When $n=$ 1 , the covering $F_{u}=\mathbb{C} \times{ }_{\mathbb{C}^{\times}} E_{u} \rightarrow E_{u}$ is not the universal covering, but the covering corresponding to the homomorphism $\pi_{1}\left(E_{u}\right) \rightarrow \mathbb{Z}$ defined by the total linking number. Then the representation of $B_{d}$ given by

$$
B_{d} \stackrel{s_{*}}{\rightarrow} \pi_{1}(\mathcal{U}) \stackrel{\tilde{\rho}}{\rightarrow} \operatorname{Aut}_{\mathbb{Z}\left[q, q^{-1}\right]}\left(H_{1}\left(F_{u} ; \mathbb{Z}\right)\right)
$$

coincides with the reduced Burau representation $([11 ; \mathrm{p} .127])$.

The complement $\Gamma^{\times} \backslash \mathcal{U}$ consists of the following two irreducible divisors :

$$
\begin{aligned}
& \mathscr{D}_{0}:=\left\{u \in \Gamma^{\times} ; \bar{X}_{u} \text { is singular }\right\}, \text { and } \\
& \mathscr{D}_{\infty}:=\left\{u \in \Gamma^{\times} ; \bar{X}_{u} \text { does } \text { not intersect } H_{\infty} \text { transversely }\right\}
\end{aligned}
$$

The main tool of the proof of Irreducibility Theorem is the Picard-Lefschetz for- 
mula, which describes the local monodromy action on $H_{n}\left(F_{b} ; \boldsymbol{Z}\right)$ along simple loops around these divisors. Roughly speaking, this formula is as follows. First, we define a boundary $\partial F_{u}$ of $F_{u}$, and an intersection pairing

$$
(,): H_{n}\left(F_{b} ; \mathbb{Z}\right) \times H_{n}\left(F_{b}, \partial F_{b} ; \mathbb{Z}\right) \rightarrow \mathbb{Z}\left[q, q^{-1}\right]
$$

in an appropriate way. Let $[\gamma] \in \pi_{1}(U, b)$ be the homotopy class of a simple loop around $\mathscr{D}_{0}$ or $\mathscr{D}_{\infty}$. Then there exists a pair of $v[\gamma] \in H_{n}\left(F_{b} ; \mathbb{Z}\right)$ and $v^{\sim}[\gamma] \in H_{n}\left(F_{b}, \partial F_{b} ; \mathbb{Z}\right)$ such that the action of $[\gamma] *$ on $H_{n}\left(F_{b} ; \mathbb{Z}\right)$ is given by

$$
x \mapsto x+\left(x, v^{\sim}[\gamma]\right) \cdot v[\gamma]
$$

This is a natural generalization of the classical Picard-Lefschetz formula with $\mathbb{Z}$ replaced by $\mathbb{Z}\left[q, q^{-1}\right]$. The homology class $v[\gamma]$ is the "vanishing cycle" associated with $[\gamma]$.

Moreover, we have the following two facts :

(1) As a module over the group ring $\mathbb{Z}\left[q, q^{-1}\right]\left[\pi_{1}(\mathcal{U}, b)\right], H_{n}\left(F_{b} ; \mathbb{Z}\right)$ is generated by one element $v\left[\gamma_{0}\right]$, where $\gamma_{0}$ is an arbitrary simple loop around $\mathscr{D}_{0}$.

(2) Let $\gamma_{\infty}$ be a simple loop around $\mathscr{D}_{\infty}$. Then there exists a simple loop $\gamma_{0}$ around $\mathscr{D}_{0}$ such that

$$
\left[\gamma_{0}\right]_{*}\left(v\left[\gamma_{\infty}\right]\right) \neq v\left[\gamma_{\infty}\right]
$$

The first fact just corresponds to the classically known fact that the space of vanishing cycles in the sense of $[8 ; \S 3]$ is generated, as a module over the group ring of the monodromy group, by one vanishing cycle for a simple loop, if the coefficients of the homology groups are in $\mathbf{Q}($ see $[8 ; \S 7])$.

On the other hand, the second fact causes the crucial difference between the classical representation $\rho$ and our representation $\widetilde{\rho}$. Indeed, for the classical monodromy $\rho(1)$, the inequality $(0.5)$ does not hold; that is, we alway have

$$
\left[\gamma_{0}\right]_{*}\left(v\left[\gamma_{\infty}\right]\right) \equiv v\left[\gamma_{\infty}\right] \quad \bmod q-1
$$

for arbitrary simple loops $\gamma_{0}$ and $\gamma_{\infty}$ around $\mathscr{D}_{0}$ and $\mathscr{D}_{\infty}$, respectively. This congruence modulo $q-1$ guarantees the stability of the subspace $H_{\mathrm{prim}}^{n-2}\left(Y_{b} ; \mathbf{Q}\right)$ of $H_{n-1}\left(X_{b} ; \mathbf{Q}\right)$ under the monodromy action, because this subspace is generated by vanishing cycles $v\left[\gamma_{\infty}\right] \bmod q-1$ associated with simple loops $\gamma_{\infty}$ around $\mathscr{D}_{\infty}$. 
The idea to look at the universal covering of the complement comes from [6]. In this paper, Givental' considered the versal deformation family of a hypersurface singularity, and studied the monodromy action on the middle homology group of the universal covering of the complement to the Milnor fiber. In the case of simple singularity, the fundamental group of the complement to the discriminant locus in the base space of the versal deformation family is known to be isomorphic to the generalized braid group corresponding to the Dynkin diagram of the simple singularity. What he obtained is a representation of the Iwahori-Hecke algebra, which connects the classical representations on the module of vanishing cycles in odd dimensions and in even dimensions.

A similar investigation had been done in [13] in a more general setting than ours.

In [11], Libgober has studied the higher homotopy groups $\pi_{n-k}\left(E_{b}\right)$ of the complements to singular hypersurfaces $X_{b}$. When $X_{b}$ has only isolated singularities, $\pi_{n}\left(E_{b}\right)$ is isomorphic to $H_{n}\left(F_{b} ; \mathbb{Z}\right)$, and a method for calculating this group via the monodromy representation arising from the Lefschetz pencil is described in $[11$; Theorem 2.4$]$.

This paper is organized as follows.

In $\S 1$, we construct the universal family of the universal coverings $F_{u} \rightarrow E_{u}$ of the complements $E_{u}=\mathbb{A}^{n} \backslash X_{u}$ over the extended base space $u \subset \Gamma^{\times}$. We shall show that the deck transformation $T_{u}: F_{u} \rightarrow F_{u}$ over $E_{u}$ corresponding to a generator of $\mathrm{Gal}\left(F_{u} / E_{u}\right) \cong \mathbb{Z}$ is also constructed universally over $\mathcal{U}$. Thus we obtain the representation $\widetilde{\rho}$.

In $\S 2$, we investigate the polynomial map $\widehat{\phi}_{u}:=f_{u} / h^{d}: \mathbb{A}^{n} \rightarrow \mathbb{C}$ which defines the affine hypersurface $X_{u}$; that is, $X_{u}=\bar{\phi}_{u}^{-1}(0)$ and $E_{u}=\widehat{\phi}_{u}^{-1}\left(\mathbb{C}^{\times}\right)$. We shall study the critical points of $\widehat{\phi}_{u}$ and the behavior of the fibers $\widehat{\phi}_{u}^{-1}(t)$ "at infinity". We introduce a Zariski open dense subset $\boldsymbol{u}_{N} \subset \mathcal{U}$, over which the topology of the polynomial maps $\widehat{\phi}_{u}$ does not vary locally.

In $\S 3$, we introduce a continuous function $\varepsilon: \mathcal{U} \rightarrow \mathbb{R}_{>0}$ which is "small enough", and define two boundaries $\partial_{0} E_{u}$ and $\partial_{\infty} E_{u}$ of $E_{u}$ as $\widehat{\phi}_{u}^{-1}\left(\Delta^{\times}(0)\right)$ and $\bar{\phi}_{u}^{-1}\left(\Delta^{\times}(\infty)\right)$, respectively, where $\Delta^{\times}(0):=\{z \in \mathbb{C}: 0<|z| \leq \varepsilon(u)\}$ and $\Delta^{\times}(\infty):=\left\{z \in \mathbb{C} ;|z|^{-1} \leq \varepsilon(u)\right\}$. We then define two boundaries $\partial_{0} F_{u}$ and $\partial_{\infty} F_{u}$ of $F_{u}$ as the pull-backs of the boundaries of $E_{u}$ by the covering map $F_{u} \rightarrow$ $E_{u}$. It turns out that the relative homology groups $H_{n}\left(F_{u}, \partial_{0} F_{u}\right)$ and $H_{n}\left(F_{u}\right.$, $\left.\partial_{\infty} F_{u}\right)$, both of which are also $\mathbb{Z}\left[q, q^{-1}\right]$-modules, are easier to investigate than $H_{n}\left(F_{u}\right)$. The pleasant feature of this theory is that there is a certain kind of duality between $H_{n}\left(F_{u}, \partial_{0} F_{u}\right)$ and $H_{n}\left(F_{u}, \partial_{\infty} F_{u}\right)$. 
In $\S 4$, we review the classical theory of Lefschetz [9], and fix some notion and notation about vanishing cycles and thimbles. In this paper, a vanishing cycle in $X_{u}$, for example, is defined as a homotopy class of continuous maps from $S^{n-1}$ to $X_{u}$ which satisfies certain conditions, and a thimble in $\left(E_{u}, \partial_{0} E_{u}\right)$, for example, is defined as a homotopy class of continuous maps from the pair $\left(C S^{n-1}, S^{n-1}\right)$, where $C S^{n-1}$ is the cone over $S^{n-1}$, to $\left(E_{u}, \partial_{0} E_{u}\right)$ which possesses certain properties.

In $\S 5$, we investigate the homology groups $H_{n-1}\left(X_{u}\right), H_{n}\left(E_{n}\right)$ and $H_{n}\left(E_{u}\right.$, $\left.\partial_{0} E_{u}\right)$. The main results are that, if $u \in U_{N}$, then the homology classes of the vanishing cycles corresponding to the critical points of $\widehat{\phi}_{u}$ form a basis of $H_{n-1}$ $\left(X_{u}\right)$, and that the homology classes of the associated thimbles form a basis of $H_{n}\left(E_{u}, \partial_{0} E_{u}\right)$. In particular, $H_{n-1}\left(X_{u}\right)$ and $H_{n}\left(E_{u}, \partial_{0} E_{u}\right)$ are canonically isomorphic, and the rank of them is equal with the number of the critical points of $\widehat{\phi}_{u}$. These facts seem to be well-known. However, we present them with complete proofs in order for the paper to be self-contained.

In $\S 6$ and $\$ 7$, we study the structure of $H_{n}\left(F_{u}\right), H_{n}\left(F_{u}, \partial_{0} F_{u}\right)$ and $H_{n}\left(F_{u}\right.$, $\left.\partial_{\infty} F_{u}\right)$. We show that $H_{n}\left(F_{u}\right)$ is embedded in $H_{n}\left(F_{u}, \partial_{0} F_{u}\right)$ and $H_{n}\left(F_{u}, \partial_{\infty} F_{u}\right)$ by the natural homomorphisms. We also show that the homology classes of the thimbles lifted from $\left(E_{u}, \partial_{0} E_{u}\right)$ (resp. $\left(E_{u}, \partial_{\infty} E_{u}\right)$ ) form a set of basis of $H_{n}\left(F_{u}\right.$, $\left.\partial_{0} F_{u}\right)$ (resp. $H_{n}\left(F_{u}, \partial_{\infty} F_{u}\right)$ ) over $\mathbb{Z}\left[q, q^{-1}\right]$. In particular, we obtain isomorphisms

$$
\begin{aligned}
& H_{n}\left(F_{u}, \partial_{0} F_{u}\right) \cong H_{n}\left(E_{u}, \partial_{0} E_{u}\right) \otimes_{\mathbf{Z}} \mathbb{Z}\left[q, q^{-1}\right] \cong H_{n-1}\left(X_{u}\right) \otimes_{\mathbf{Z}} \mathbb{Z}\left[q, q^{-1}\right], \text { and } \\
& H_{n}\left(F_{u}, \partial_{\infty} F_{u}\right) \cong H_{n}\left(E_{u}, \partial_{\infty} E_{u}\right) \otimes_{\mathbf{Z}} \mathbb{Z}\left[q, q^{-1}\right]
\end{aligned}
$$

These isomorphisms are, however, not canonical by any means, because there is ambiguity in the way how to lift a given thimble in $\left(E_{u}, \partial_{0} E_{u}\right)$ (resp. $\left(E_{u}\right.$, $\left.\left.\partial_{\infty} E_{u}\right)\right)$ to $\left(F_{u}, \partial_{0} F_{u}\right)$ (resp. $\left.\left(F_{u}, \partial_{\infty} F_{u}\right)\right)$. In order to state the isomorphisms (0.6) precisely, we have to restrict ourselves to a smaller locus $\boldsymbol{U}_{N} \subset \mathcal{U}_{N}$, over which a canonical lifting can be assigned to each thimble in $\left(E_{u}, \partial_{0} E_{u}\right)$ or in $\left(E_{u}, \partial_{\infty} E_{u}\right)$. However, $\mathcal{U} \backslash \mathcal{U}_{N}^{\tilde{N}}$ is a real semi-algebraic subset of real codimension 1 , and the homomorphism $\pi_{1}\left(\mathcal{U}_{N}^{\tilde{N}}\right) \rightarrow \pi_{1}(U)$ induced by the inclusion is not surjective. Hence these isomorphisms cannot be $\pi_{1}(\mathcal{U})$-equivariant. (Otherwise, we would get a contradiction to Irreducibility Theorem above.)

In $\S 8$, we introduce two intersection pairings between the two relative homology groups $H_{n}\left(F_{u}, \partial_{0} F_{u}\right)$ and $H_{n}\left(F_{u}, \partial_{\infty} F_{u}\right)$, which take values in $\mathbb{Z}\left[q, q^{-1}\right]$, and prove that they are non-degenerate. The idea of these pairings is also due to $[6]$. 
In $\S 9$, we formulate and state the Picard-Lefschetz formula. Let $\gamma_{0}$ be a simple loop around $\mathscr{D}_{0}$, and $\gamma_{\infty}$ a simple loop around $\mathscr{D}_{\infty}$. The precise definition of simple loops is given in $\S 9.1$. We describe the action of $\left[\gamma_{0}\right] \in \pi_{1}(U, b)$ on $H_{n}\left(F_{b}, \partial_{\infty} F_{b}\right)$ in Theorem 9.2.1, and the action of $\left[\gamma_{\infty}\right] \in \pi_{1}(U, b)$ on $H_{n}\left(F_{b}\right.$, $\left.\partial_{0} F_{b}\right)$ in Theorem 9.2.2, with the help of the "hermitian" intersection pairings defined in $\S 8$. As is seen from the proofs, which are given in $\S 9.4$ and $\S 9.7$ respectively, this is a more appropriate way to state Picard-Lefschetz formula than to describe the action on $H_{n}\left(F_{b}\right)$. The action on $H_{n}\left(F_{b}\right)$, however, can be derived from these two theorems, because $H_{n}\left(F_{b}\right)$ is embedded in $H_{n}\left(F_{b}, \partial_{\infty} F_{b}\right)$ and $H_{n}\left(F_{b}, \partial_{0} F_{b}\right)$ by the natural homomorphisms.

As can be guessed from the fact that, for $b \in U_{N}$, the basis of $H_{n}\left(F_{b}, \partial_{\infty} F_{b}\right)$ or $H_{n}\left(F_{b}, \partial_{0} F_{b}\right)$ over $\mathbb{Z}\left[q, q^{-1}\right]$ consists of the homology classes of lifted thimbles, each of which corresponds to a critical value of $\bar{\phi}_{b}$ in a bijective way, the main ingredient of the proof is to study the movements of the critical values of $\widehat{\phi}_{u}$ when $u$ makes a round trip along $\gamma_{0}$ or $\gamma_{\infty}$. In the case of $\gamma_{0}$, it is quite easy to see how the critical values moves on the complex plane. On the contrary, it takes the whole subsection $\S 9.6$ in the case of $\gamma_{\infty}$.

There is one more important result in $\S 9$. In $\S 9.5$, we give a proof of Theorem 9.5.1, which states that $H_{n}\left(F_{b}\right)$ is generated, as a module over the group ring $\mathbb{Z}\left[q, q^{-1}\right]\left[\pi_{1}(\mathcal{U}, b)\right]$, by one "vanishing cycle" $v\left[\gamma_{0}\right]$ associated with an arbitrary simple loop $\gamma_{0}$ around $\mathscr{D}_{0}$.

By Zariski's hyperplane section theorem, $\pi_{1}(U)$ is generated by the homotopy classes of simple loops around $\mathscr{D}_{0}$ and $\mathscr{D}_{\infty}$. Hence, using the results in $\S 9$, we can prove Irreducibility Theorem in $\S 10$.

\section{Acknowledgment}

The author thanks Professor A. Libgober for his valuable comments. He also thanks Max-Planck-Institut für Mathematik in Bonn for providing him with stimulating research environment.

\section{Conventions}

(1) The symbol $I$ always denotes the closed interval $[0,1] \subset \mathbb{R}$.

(2) A path $I \rightarrow V$ on a $\mathscr{C}^{\infty}$-manifold $V$ is always assumed to be piece-wise smooth.

(3) Let $\alpha: I \rightarrow V$ and $\beta: I \rightarrow V$ be two paths on a topological space $V$. We define the order of the product of paths in such a way that $\alpha \cdot \beta$ is well-defined if and only if $\beta(1)=\alpha(0)$ :

(4) Let $V_{1}$ and $V_{2}$ be two topological spaces, or two pairs of topological spaces. Then $\left[V_{1}, V_{2}\right]$ denotes the set of homotopy classes of continuous maps 
from $V_{1}$ to $V_{2}$.

(5) Let $V, W$ and $W^{\prime}$ be topological spaces, and $f: V \rightarrow W, g: W^{\prime} \rightarrow W$ continuous maps. We say that $f$ is locally trivial over $g: W^{\prime} \rightarrow W$ (or simply over $W^{\prime}$ ) if the pull-back $W^{\prime} \times{ }_{W} V \rightarrow W^{\prime}$ of $f$ by $g$ is locally trivial.

(6) Let $X_{1}$ and $X_{2}$ be complex manifolds, and let $h: X_{1} \rightarrow X_{2}$ be a holomorphic map. We say that $h$ is locally trivial if it is locally trivial in the category of topological spaces and continuous maps.

(7) In this paper, we work with homology groups with coefficients $\mathbb{Z}$ unless otherwise stated, and we omit $\mathbb{Z}$ in the notation.

\section{\$1. Construction of the Universal Family}

We choose $h \in \Gamma\left(\mathbb{P}^{n}, \mathcal{O}(1)\right)$ which defines the hyperplane $H_{\infty}=\{h=0\}$, and fix it throughout this paper. Then $h^{d} \in \Gamma^{\times}$. Recall that $f_{u}$ denote the homogeneous polynomial of degree $d$ corresponding to $u \in \Gamma^{\times}$. Using the fixed homogeneous polynomial $h^{d}$, we get a morphism

$$
\phi_{u}:=f_{u} / h^{d}: E_{u} \rightarrow \mathbb{C}^{\times}
$$

which is the restriction of the polynomial map

$$
\widehat{\phi}_{u}:=f_{u} / h^{d}: \mathbb{A}^{n} \rightarrow \mathbb{C}
$$

to $E_{u}=\widehat{\phi}_{u}^{-1}\left(\mathbf{C}^{\times}\right)$. The following lemma is easy to prove by using Zariski's hyperplane section theorem $([14],[7])$, and the theorem of Fulton-Deligne on Zariski's conjecture ([3], [4], [5]).

Lemma 1.1. Suppose that $u \in \mathcal{U}$. Then $\bar{\phi}_{u}$ induces an isomorphism $\pi_{1}\left(E_{u}\right)$ $\cong \pi_{1}\left(\mathbb{C}^{\times}\right)$on the fundamental groups.

Let $e: \mathbb{C} \rightarrow \mathbb{C}^{\times}$be the universal covering given by $z \mapsto \exp z$. For every $u$ $\in \Gamma^{\times}$, we define a complex manifold $F_{u}$ by the fiber product

$$
\begin{array}{rll} 
& \tilde{e} & \\
F_{u} & \rightarrow & E_{u} \\
\phi_{u} \downarrow & \square & \downarrow \phi_{u} \\
\mathbf{C} & \rightarrow & \mathbf{C}^{\times} \\
& e &
\end{array}
$$


If $u \in U$, then Lemma 1.1 implies that the covering $\tilde{e}: F_{u} \rightarrow E_{u}$ is the universal covering of $E_{u}$ whose Galois group is canonically isomorphic to $\pi_{1}\left(\mathbb{C}^{\times}\right)$. Let $T_{u}: F_{u} \rightarrow F_{u}$ denote the deck transformation over $E_{u}$ corresponding to the counter-clockwise generator of $\pi_{1}\left(\mathbb{C}^{\times}\right)$.

The construction of the universal covering $F_{u} \rightarrow E_{u}$ can be carried out universally over the base space $\mathcal{U}$. Let $\mathscr{X} \subset \mathbb{A}^{n} \times \mathcal{U}$ denote the universal family of the affine hypersurfaces $\left\{X_{u} ; u \in \mathscr{U}\right\}$ with the natural projection $\mathscr{H} \rightarrow \mathscr{U}$, and let $\mathscr{E}$ stand for the complement $\left(\mathbb{A}^{n} \times \mathscr{U}\right) \backslash \mathscr{X}$, which is the universal family of $\left\{E_{u}\right.$; $u \in \mathcal{U}\}$ with the natural projection $\mathscr{E} \rightarrow \mathcal{U}$. By putting $\phi_{u}: E_{u} \rightarrow \mathbb{C}^{\times}$together, we get a morphism $\mathscr{E} \rightarrow \mathbb{C}^{\times}$, which maps $(P, u) \in \mathscr{E}$ to $\phi_{u}(P) \in \mathbb{C}^{\times}$. Let $\mathscr{F}$ be the fiber product $\mathscr{E} \times{ }_{\mathbb{C} \times} \mathbb{C}$, where $\mathbb{C} \rightarrow \mathbb{C}^{\times}$is given by the exponential map $e$. Then this $\mathscr{F}_{F}$ with the natural projection onto $U$ is the universal family of $\left\{F_{u} ; u\right.$ $\in \mathcal{U}$ \}. Again, the natural map $\mathscr{F} \rightarrow \mathscr{E}$ is the Galois covering with the Galois group canonically isomorphic to $\pi_{1}\left(\mathbb{C}^{\times}\right)$. Let $\mathscr{T}: \mathscr{F} \rightarrow \mathscr{F}$ be the deck transformation over $\mathscr{E}$ corresponding to the counter-clockwise generator of $\pi_{1}\left(\mathbb{C}^{\times}\right)$. Then the restriction of $\mathscr{T}$ to a fiber $F_{u} \subset \mathscr{F}$ over $u \in U$ gives the deck transformation $T_{u}: F_{u} \rightarrow F_{u}$.

Now it is easy to see tha the families $\mathscr{X} \rightarrow \boldsymbol{U}, \mathscr{E} \rightarrow \mathcal{U}$ and hence $\mathscr{F} \rightarrow \boldsymbol{U}$ are all locally trivial. Therefore we obtain a natural monodromy representation of $\pi_{1}(\mathcal{U}, b)$ on $H_{n}\left(F_{b}\right)$, where $b \in \mathcal{U}$ is a base point. Since the deck transformations $T_{u}$ are defined globally over $\mathcal{U}$, we get the following:

Lemma $\mathbb{1}_{0}$. The monodromy action of $\pi_{1}(U, b)$ on $H_{n}\left(F_{b}\right)$ commutes with the automorphism $T_{b *}: H_{n}\left(F_{b}\right) \rightarrow H_{n}\left(F_{b}\right)$ induced by the deck transformation.

We fix an isomorphism between the group ring $\mathbb{Z}\left[\pi_{1}\left(\mathbb{C}^{x}\right)\right]$ and the ring of Laurent polynomials $\mathbb{Z}\left[q, q^{-1}\right]$ by identifying the counter-clockwise generator of $\pi_{1}\left(\mathbb{C}^{\times}\right)$with $q$. Then $H_{n}\left(F_{u}\right)$ becomes a $\mathbb{Z}\left[q, q^{-1}\right]$-module for each $u \in \mathcal{U}$, in which the multiplication by $q$ is nothing but the automorphism $T_{u^{*}}: H_{n}\left(F_{u}\right) \rightarrow$ $H_{n}\left(F_{u}\right)$. Lemma 1.2 implies that the monodromy representation of $\pi_{1}(u, b)$ on $H_{n}\left(F_{b}\right)$ is a representation on the $\mathbb{Z}\left[q, q^{-1}\right]$-module, and thus we get

$$
\pi_{1}(\mathcal{U}, b) \rightarrow \operatorname{Aut}_{\mathbb{Z}\left[q, q^{-1}\right]}\left(H_{n}\left(F_{b}\right)\right)
$$

This monodromy representation is the central theme of this article.

The natural projection $U \rightarrow U$ is a $\mathbb{C}^{\times}$-bundle. Hence the kernel of $\pi_{1}(\mathcal{U})$ $\rightarrow \pi_{1}(U)$ is generated by an element $\mathfrak{c} \in \pi_{1}(U)$, which is represented by a counter-clockwise loop in the fiber $\cong \mathbb{C}^{\times}$. It is obvious that $c$ is contained in the center of $\pi_{1}(\mathcal{U})$. 
Proposition 1.1. The action of $\mathfrak{c}$ on $H_{n}\left(F_{b}\right)$ is equal with the multiplication by $q$.

Proof. The homotopy class $c \in \pi_{1}(\mathcal{U}, b)$ is represented by the loop $\gamma: I \rightarrow$ $\mathcal{U}$ given by $f_{r(t)}=e^{2 \pi \sqrt{-1} t} f_{b}$. The complement $E_{\gamma(t)}$ does not move in $\mathbf{A}^{n}$ even when $t$ varies. The function $\phi_{\gamma(t)}=f_{\gamma(t)} / h^{d}: E_{\gamma(t)} \rightarrow \mathbb{C}^{\times}$on $E_{\gamma(t)}$, however, varies as $\phi_{\gamma(t)}=e^{2 \pi \sqrt{-1} t} \phi_{b}$. This is equivalent to rotate $E_{b}$ over $\mathbb{C}^{\times}$once in the counter-clockwise direction. Therefore it induces the deck transformation $T_{b}$ on $F_{b}$, and hence the multiplication by $q$ on $H_{n}\left(F_{b}\right)$.

This proposition shows that the family $\mathscr{F} \rightarrow \mathcal{U}$ is not a pull-back of any family over $U$, and hence justifies us in working, not with $\pi_{1}(U)$, but with $\pi_{1}(\mathcal{U})$. Later on, we shall prove that $H_{n}\left(F_{b}\right)$ is torsion free as a $\mathbb{Z}\left[q, q^{-1}\right]$-module (Corollary 6.1). Hence $\mathfrak{c}$ has an infinite order in $\pi_{1}(\mathcal{U}, b)$. Therefore we have the following:

Corollary 1.1. The fundamental group $\pi_{1}(\mathcal{U}, b)$ is a central extension of $\pi_{1}(U, b)$ by $\mathbb{Z}$.

\section{\$2. Structure of the Polynomial $\operatorname{Map} \widehat{\phi}_{u}$}

The complement $\mathbb{P}_{*}(\Gamma) \backslash U$ consists of two irreducible divisors $D_{0}$ and $D_{\infty}$, where $D_{0}$ consists of all singular hypersurfaces, while $D_{\infty}$ consists of all hypersurfaces whose intersections with $H_{\infty}$ are not transverse. Then a general point of $D_{0}$ corresponds to a hypersurface possessing one ordinary double point as its only singularities, while a general point of $D_{\infty}$ corresponds to a non-singular hypersurface $\bar{X}$ such that $H_{\infty} \cap \bar{X}$ is a hypersurface in $H_{\infty}$ possessing only one ordinary double point as its singularities.

Then the divisors $\mathscr{D}_{0}$ and $\mathscr{D}_{\infty}$ of $\Gamma^{\times}$defined in Introduction are the pull-backs of $D_{0}$ and $D_{\infty}$, respectively, by the natural projection $\mathrm{pr}: \Gamma^{\times} \rightarrow$ $\mathbf{P}_{*}(\Gamma)$.

We write by $\mathfrak{h}_{\infty} \in \mathbb{P}_{*}(\Gamma)$ the point corresponding to the multiple hyperplane $d \cdot H_{\infty}$. In this section, we always assume $u \notin \operatorname{pr}^{-1}\left(\mathfrak{h}_{\infty}\right)$, so that $\bar{\phi}_{u}: \mathbb{A}^{n}$ $\rightarrow \mathbf{C}$ is not a constant map.

Let $\operatorname{Cr}(u) \subset \mathbf{C}$ denote the set of critical values of $\widehat{\phi}_{u}$. By definition, we have 


$$
t \notin \operatorname{Cr}(u) \Leftrightarrow \widehat{\phi}_{u}^{-1}(t) \text { is non-singular. }
$$

In particular, $u \in \mathscr{U}$ implies $0 \notin \mathrm{Cr}(u)$.

Let $\mathscr{L}_{u} \subset \Gamma^{\times}$denote the affine line $\left\{f_{u}-t \cdot h^{d} ; t \in \mathbb{C}\right\}$, and let $L_{u} \subset \mathbb{P}_{*}(\Gamma)$ denote the projective line spanned by $\mathfrak{h}_{\infty}$ and the point $\operatorname{pr}(u) \in \mathbb{P}_{*}(\Gamma)$. We put

$$
L_{u}^{o}:=L_{u} \backslash\left\{\mathfrak{h}_{\infty}\right\} .
$$

Then the projection pr: $\Gamma^{\times} \rightarrow \mathbb{P}_{*}(\Gamma)$ induces an isomorphism between $\mathscr{L}_{u}$ and $L_{u}^{o}$. There are natural parameterizations

$$
\iota_{u}: \stackrel{\mathbb{C}}{\rightarrow} \mathscr{L}_{u}, \quad \text { and } \quad \bar{\iota}_{u}:=\operatorname{pro}_{u}: \stackrel{\mathbb{C}}{\rightarrow} L_{u}^{o}
$$

given by $\iota_{u}(t):=f_{u}-t \cdot h^{d}$. Note that the assumption $u \notin \operatorname{pr}^{-1}\left(\mathfrak{h}_{\infty}\right)$ implies $w$ $\notin \operatorname{pr}^{-1}\left(\mathfrak{h}_{\infty}\right)$ for all $w \in \mathscr{L}_{u}$. The following remark will be used frequently throughout this paper.

Remark 2.1. By definition, the morphism $\widehat{\phi}_{u}: \mathbb{A}^{n} \rightarrow \mathbb{C}$ is nothing but the pull-back of the universal family $\mathscr{x}_{\Gamma} \rightarrow \Gamma^{\mathrm{x}}$ by

$$
\underset{\mathbb{C}}{\stackrel{\sim}{\rightarrow}} \mathscr{L}_{u} \hookrightarrow \Gamma^{\times},
$$

where $\mathscr{X}_{\Gamma}:=\left\{(P, u) \in \mathbb{A}^{n} \times \Gamma^{\times} ; P \in X_{u}\right\}$, and $\mathscr{X}_{\Gamma} \rightarrow \Gamma^{\times}$is the second projection.

Proposition 2.1. If $u \in \mathcal{U}$, then $\bar{\phi}_{u}: \mathbb{A}^{n} \rightarrow \mathbb{C}$ is locally trivial over $\mathbb{C} \backslash \mathrm{Cr}(u)$.

Proof. By (2.1), it is enough to show that $\bar{\phi}_{u}$ is locally trivial "at infinity" over the complex plane $\mathbb{C}$; that is, if $u \in U$, then, for all $t \in \mathbb{C}$, the projective compactification of the affine hypersurface $\widehat{\phi}_{u}^{-1}(t)$ is non-singular at every point of the intersection with $H_{\infty}$, and moreover, the intersection is transverse. This follows directly from two Lemmas below and Remark 2.1

Note that 


$$
\bar{X}_{w} \cap H_{\infty}=\bar{X}_{u} \cap H_{\infty} \text { for all } w \in \mathscr{L}_{u},
$$

by the definition of $\mathscr{L}_{u}$.

Lemma 2.1. Suppose that $\bar{X}_{w}$ is non-singular at a point $P \in \bar{X}_{w} \cap H_{\infty}$ for one $w \in \mathscr{L}_{u}$. Then $\bar{X}_{w^{\prime}}$ is non-singular at $P$ for all $w^{\prime} \in \mathscr{L}_{u}$.

Lemma 2.2. Suppose that $\bar{X}_{w}$ intersects $H_{\infty}$ transversely at a point $P \in \bar{X}_{w}$ $\cap H_{\infty}$ for one $w \in \mathscr{L}_{u}$. Then $\bar{X}_{w^{\prime}}$ intersects $H_{\infty}$ transversely at $P$ for all $w^{\prime} \in \mathscr{L}_{u}$.

Proofs of Lemmas 2.1 and 2.2. Let $\left(z_{1}, \ldots, z_{n}\right)$ be an affine coordinate system on an affine open subset $\left(\mathbb{A}^{n}\right)^{\prime}$ of $\mathbb{P}^{n}$ with the origin $P$ such that $H_{\infty}=\left\{z_{n}=0\right\}$. Suppose that $\bar{X}_{u}$ is defined by

$$
f_{u}\left(z_{1}, \ldots, z_{n}\right)=0
$$

in $\left(\mathbf{A}^{n}\right)^{\prime}$, where $f_{u}\left(z_{1}, \ldots, z_{n}\right)$ is an inhomogeneous polynomial with zero constant term. If $w=\iota_{u}(t)$, then, after replacing $z_{n}$ with $\alpha z_{n}$ where $\alpha$ is an appropriate non-zero constant, an inhomogeneous polynomial defining $\bar{X}_{w}$ is given by

$$
f_{w}\left(z_{1}, \ldots, z_{n}\right):=f_{u}\left(z_{1}, \ldots, z_{n}\right)-t \cdot z_{n}^{d}
$$

The projective hypersurface $\bar{X}_{w}$ is non-singular at $P$ if and only if the homogeneous part $f_{w}^{[1]}\left(z_{1}, \ldots, z_{n}\right)$ of degree 1 in $f_{w}\left(z_{1}, \ldots, z_{n}\right)$ is non-zero. Since $d \geq$ 2 , if it holds for one $w \in \mathscr{L}_{u}$, then it holds for all $w \in \mathscr{L}_{u}$. The condition that the intersection of $\bar{X}_{w}$ and $H_{\infty}$ is transverse at $P$ is equivalent to the condition that $f_{w}^{[1]}\left(z_{1}, \ldots, z_{n-1}, 0\right)$ is non-zero. Again, if it holds for one $w \in \mathscr{L}_{u}$, then it holds for all $w \in \mathscr{L}_{u}$.

These two lemmas imply the following two propositions.

Proposition 2.2. If $u \notin \mathscr{D}_{\infty}$, then $\mathscr{L}_{u} \cap \mathscr{D}_{\infty}=\emptyset$. If $u \in \mathscr{D}_{\infty}$, then $\mathscr{L}_{u} \subset$ $\mathscr{D}_{\infty}$.

This implies that $D_{\infty} \subset \mathbf{P}_{*}(\Gamma)$ has a structure of the cone with the vertex $\mathfrak{h}_{\infty}$.

We have an inclusion $\operatorname{Cr}(u) \subset \iota_{u}^{-1}\left(\mathscr{L}_{u} \cap \mathscr{D}_{0}\right)=\bar{\iota}_{u}^{-1}\left(L_{u}^{o} \cap D_{0}\right)$ from (2.1) and Remark 2.1. 
Proposition 2.3. If $\bar{X}_{u}$ is non-singular at every point of $\bar{X}_{u} \cap H_{\infty}$, then $\operatorname{Cr}(u) \subset \mathbb{C}$ is equal with $\iota_{u}^{-1}\left(\mathscr{L}_{u} \cap \mathscr{D}_{0}\right)$ and with $\bar{\ell}_{u}^{-1}\left(L_{u}^{o} \cap D_{0}\right)$.

Corollary 2.1. If $u \in \mathcal{U}$, then $\mathscr{L}_{u} \cap \mathscr{D}_{\infty}=\emptyset$, and $\operatorname{Cr}(u)=\iota_{u}^{-1}\left(\mathscr{L}_{u} \cap \mathscr{D}_{0}\right)$.

Let $\widehat{\phi}_{u}\left(x_{1}, \ldots, x_{n}\right)$ be the polynomial expressing $\widehat{\phi}_{u}: \mathbb{A}^{n} \rightarrow \mathbb{C}$ in terms of affine coordinates $\left(x_{1}, \ldots, x_{n}\right)$ of $\mathbb{A}^{n}$. The critical points of $\widehat{\phi}_{u}$ are then given by the solutions of

$$
\frac{\partial \widehat{\phi}_{u}}{\partial x_{1}}=\cdots=\frac{\partial \widehat{\phi}_{u}}{\partial x_{n}}=0
$$

Hence, if $u \in \mathcal{U}$ is chosen generally, the number of the distinct critical points of $\widehat{\phi}_{u}$ is

$$
N:=(d-1)^{n}
$$

Definition 2.1. Let $U_{N} \subset \mathcal{U}$ denote the locus of all $u \in U$ which satisfies the following :

(i) $\operatorname{Cr}(u)$ consists of distinct $N$ values, and (ii) over each $p \in \operatorname{Cr}(u), \widehat{\phi}_{u}$ has only one critical point and this critical point is non-degenerate.

Since both of (i) and (ii) are algebraically open conditions, the locus $\boldsymbol{U}_{N}$ is a Zariski open subset of $U$. It is easy to see that $u_{N} \neq \emptyset$. Hence $U_{N} \subset \mathcal{U}$ is dense.

Note that $N$ is the maximal number which can be attained by the number of elements of $\mathrm{Cr}(u)$. Hence Corollary 2.1 implies the following:

Proposition 2.4. If $u \in \mathcal{U}_{N}$, then $\mathscr{L}_{u}$ intersects $\mathscr{D}_{0}$ at distinct $N$ points of the non-singular locus of $\mathscr{D}_{0}$ transversely.

Lemma 2. $\mathscr{B}_{\text {。 }}$ Let $u$ be a point of $\mathscr{U}_{N}$. Then we have $\mathscr{L}_{u} \backslash \mathscr{D}_{0}=\mathscr{L}_{u} \cap \mathcal{U}=\mathscr{L}_{u}$ $\cap \mathcal{U}_{N}$

Proof. Let $w$ be an arbitrary point of $\mathscr{L}_{u}$. By definition, the affine line $\mathscr{L}_{w}$ is equal with $\mathscr{L}_{u}$, and we write this affine line simply by $\mathscr{L}$. By Remark 2.1, we have

$$
\iota_{u} \circ \bar{\phi}_{u}=\iota_{w} \circ \bar{\phi}_{w}
$$


as a morphism from $\mathrm{A}^{n}$ to $\mathscr{L}$; that is, $\widehat{\phi}_{u}$ and $\widehat{\phi}_{w}$ differ only by translation of C. In particular, the morphism $\widehat{\phi}_{w}$ also satisfies the conditions (i) and (ii) in Definition 2.1. This implies that, if $w \in \mathcal{U}$, then $w \in \mathcal{U}_{N}$. On the other hand, because of Corollary 2.1, we have $\mathscr{L} \cap \mathscr{D}_{\infty}=\emptyset$ and hence $\mathscr{L} \backslash \mathscr{D}_{0}=\mathscr{L} \cap \mathcal{U}=\mathscr{L} \cap$ $u_{N}$.

Suppose that $u \in U_{N}$. Let $p \in \mathrm{C}$ be one of the critical values of $\bar{\phi}_{u}$, and let $q \in \mathbf{A}^{n}$ be the critical point of $\widehat{\phi}_{u}$ on $\widehat{\phi}_{u}^{-1}(p)$. Then there exists an analytically local coordinate system $\left(w_{1}, \ldots, w_{n}\right)$ on a small neighborhood of $q$ in $\mathbf{A}^{n}$ with the center $q$ such that $\widehat{\phi}_{u}$ is given by

$$
\left(w_{1}, \ldots, w_{n}\right) \mapsto p+w_{1}^{2}+\cdots+w_{n}^{2}
$$

locally around $q$. Let $\epsilon$ be a small positive real number. We put

$$
B:=\left\{\left(w_{1}, \ldots, w_{n}\right) ;\left|w_{1}\right|^{2}+\cdots+\left|w_{n}\right|^{2} \leq \epsilon\right\} \subset \mathbf{A}^{n}
$$

Let Int $B$ be the interior of $B$. Lemmas 2.1 and 2.2 imply the following (cf. [8 ; §3. Ehresmann's Fibration Theorem]):

Proposition 2.5. Let $\eta$ be a positive real number small enough compared with $\epsilon$, and let $\Delta \subset \mathrm{C}$ be the closed disk with the center $p$ and of radius $\eta$. (1) By the restriction of $\bar{\phi}_{u}$, the pair $\left(\phi_{u}^{-1}(\Delta) \backslash\right.$ Int $\left.B, \phi_{u}^{-1}(\Delta) \cap \partial B\right)$ is a trivial fiber space with boundary over $\Delta$. (2) Moreover, $\phi_{u}^{-1}(p)$ is a strong deformation retract of $\phi_{u}^{-1}(\Delta)$.

Proof. The situation near $H_{\infty}$ can be checked by Lemmas 2.1 and 2.2. The situation near the point $q$ can be studied by the explicit formula (2.4) of $\widehat{\phi}_{u}$.

Construction 2.1. The critical values and the critical points of $\bar{\phi}_{u}$ define multivalued functions from $\boldsymbol{U}_{N}$ to $\mathbf{C}$ and $\mathbf{A}^{n}$, respectively. In order to make them single-valued, and to study their behavior near a point of $\Gamma^{\times} \backslash \mathcal{U}_{N}$, we make the following construction of a curve $C$ and morphisms $\tilde{p}_{i}, \tilde{q}_{i}$.

Suppose that we are given the following data $(\mathscr{A}, c, b, \Delta)$ :

A point $c$ of $\Gamma^{\times}$, an affine line $\mathscr{A} \subset \Gamma$ passing through $c$ such that $\mathscr{A} \cap \mathscr{U}_{N} \neq \emptyset$, a sufficiently small closed disk $\Delta$ on $\mathscr{A}$ with the center $c$, and a base point $b$ on the boundary $\partial \Delta$. Since $\Delta$ is small enough and $\mathscr{A} \cap U_{N} \neq \emptyset$, we may assume that

$$
\Delta \backslash\{c\} \subset \mathcal{U}_{N}
$$


We consider the punctured affine line $\mathscr{A} \cap \mathscr{U}_{N}$. The critical values and the critical points of $\widehat{\phi}_{u}$ yield multi-valued algebraic functions on $\mathscr{A} \cap \boldsymbol{U}_{N}$ to $\mathbb{C}$ and to $\mathbb{A}^{n}$, respectively. Let $W$ be a simply-connected small open neighborhood of $b$ in $\mathscr{A} \cap \mathscr{U}_{N}$. The critical values and the critical points become single-valued when $u$ is restricted to move in $W$. Let

$$
p_{\imath}: W \rightarrow \mathbb{C}, \quad \text { and } \quad q_{\imath}: W \rightarrow \mathbb{A}^{n} \quad(i=1, \ldots, N)
$$

denote those single-valued functions on $W$ such that the critical point $q_{t}(u) \in$ $\mathbb{A}^{n}$ of $\widehat{\phi}_{u}$ is mapped to $p_{v}(u) \in \mathbb{C}$ by $\widehat{\phi}_{u}: \mathbb{A}^{n} \rightarrow \mathbb{C}$ for all $u \in W$. The fundamental group $\pi_{1}\left(\mathscr{A} \cap \mathcal{U}_{N}, b\right)$ acts on the set $\operatorname{Cr}(b)$, and hence we get a natural homomorphism

$$
m: \pi_{1}\left(\mathscr{A} \cap \mathcal{U}_{N}, b\right) \rightarrow \mathfrak{S}(\mathrm{Cr}(b))
$$

where $\mathfrak{S}(\operatorname{Cr}(b))$ is the permutation group of the set $\operatorname{Cr}(b)$. Let

$$
\rho^{\prime}: C^{\prime} \rightarrow \mathscr{A} \cap \mathscr{U}_{N}
$$

be the finite étale Galois covering corresponding to $m$. The Galois group is isomorphic to the image of $m$. We choose a base point $b^{\prime} \in C^{\prime}$ such that $\rho^{\prime}\left(b^{\prime}\right)=$ $b$. Let $W^{\prime} \subset C^{\prime}$ be the unique connected component of $\rho^{\prime-1}(W)$ which contains the base point $b^{\prime}$. Then there exist single-valued algebraic functions

$$
p_{i}^{\prime}: C^{\prime} \rightarrow \mathbb{C} \text { for } i=1, \ldots . N
$$

such that $p_{i}^{\prime}\left(w^{\prime}\right)=p_{1}\left(\rho^{\prime}\left(w^{\prime}\right)\right)$ for $w^{\prime} \in W^{\prime}$, and there also exist algebraic morphisms

$$
q_{\imath}^{\prime}: C^{\prime} \rightarrow \mathbb{A}^{n} \quad \text { for } \quad i=1, \ldots, N
$$

such that $q_{i}^{\prime}\left(w^{\prime}\right)=q_{i}\left(\rho^{\prime}\left(w^{\prime}\right)\right)$ for $w^{\prime} \in W^{\prime}$. The $p_{i}^{\prime}$ and the $q_{i}^{\prime}$ are determined uniquely because $C^{\prime}$ is connected. Let

$$
\rho: C \rightarrow \mathscr{A}
$$

be the finite morphism extending the étale covering $\rho^{\prime}: C^{\prime} \rightarrow \mathscr{A} \cap \mathcal{U}_{N}$. Since $q_{i}^{\prime}$ and $p_{\imath}^{\prime}$ are algebraic morphisms, they can be extended to the morphisms

$$
\tilde{q}_{i}: C \rightarrow \mathbb{P}^{n}=\mathbb{A}^{n} \cup H_{\infty} \text { and } \tilde{p}_{i}: C \rightarrow \mathbb{P}^{1}=\mathbb{C} \cup\{\infty\}
$$


in a unique way. By the construction, if $w \in \rho^{-1}\left(\mathscr{A} \cap \mathcal{U}_{N}\right)$, then $\left\{\tilde{q}_{1}(w), \ldots\right.$, $\left.\tilde{q}_{N}(w)\right\}$ is the set of critical points of $\widehat{\phi}_{\rho(w)}$, and $\tilde{p}_{i}(w)=\widehat{\phi}_{\rho(w)}\left(\tilde{q}_{i}(w)\right)$ holds for $i$ $=1, \ldots, N$.

Construction 2.2. Let $\widetilde{\Delta}$ be the connected component of $\rho^{-1}(\Delta)$ containing $b^{\prime}$. Then there exists a unique point $\tilde{c} \in \widetilde{\Delta}$ such that $\rho(\tilde{c})=c$. The behavior of critical values and critical points near the point $c$ can be investigated by looking at the morphisms $\tilde{p}_{i}$ and $\tilde{q}_{2}$ around $\tilde{c}$. We will be interested in the case where one of the critical values tends to $\infty$, or one of the critical points tends to $H_{\infty}$, when $u$ approaches $c$.

We choose an affine subspace $\left(\mathbf{A}^{n}\right)^{\prime}$ of $\mathbb{P}^{n}$ which contains $\tilde{q}_{2}(\tilde{c}), \ldots, \tilde{q}_{N}(\tilde{c})$ and satisfies $\left(\mathbb{A}^{n}\right)^{\prime} \cap H_{\infty} \neq \emptyset$. Let $\left(z_{1}, \ldots, z_{n}\right)$ be affine coordinates on $\left(\mathbb{A}^{n}\right)^{\prime}$ such that $H_{\infty}=\left\{z_{n}=0\right\}$. Since $\Delta$ is located on the affine line $\mathscr{A}$, the homogeneous polynomial $f_{u} \in \Gamma$ corresponding to $u \in \Delta$ is written in the form

$$
f_{u}=f_{c}+t(u) \cdot g
$$

where $t: \mathscr{A} \rightarrow \mathbb{C}$ is an affine coordinate such that $t(c)=0$, and $g$ is a certain polynomial in $\Gamma$. By abuse of notation, we denote by $f_{c}\left(z_{1}, \ldots, z_{n}\right)$ and $g\left(z_{1}, \ldots, z_{n}\right)$ the inhomogeneous polynomials associated to $f_{c}$ and $g$, respectively. Note that they are determined uniquely only up to multiplications by non-zero constants. By choosing them suitably, we can write the rational function $\widehat{\phi}_{u}=f_{u} / h^{d}$ on $\left(\mathbb{A}^{n}\right)^{\prime}$ in the following form for $u \in \Delta$ :

$$
\widehat{\phi}_{u}=\frac{f_{u}\left(z_{1}, \ldots, z_{n}\right)}{z_{n}^{d}}=\frac{f_{c}\left(z_{1}, \ldots, z_{n}\right)+t(u) \cdot g\left(z_{1}, \ldots, z_{n}\right)}{z_{n}^{d}}
$$

We define polynomials $h_{1}\left(u ; z_{1}, \ldots, z_{n}\right), \ldots, h_{n}\left(u ; z_{1}, \ldots, z_{n}\right)$ in $z_{1}, \ldots, z_{n}$ as follows :

$$
\begin{aligned}
& h_{i}\left(u ; z_{1}, \ldots, z_{n}\right):=z_{n}^{d} \frac{\partial \widehat{\phi}_{u}}{\partial z_{\imath}}=\frac{\partial f_{u}\left(z_{1}, \ldots, z_{n}\right)}{\partial z_{\imath}} \text { for } i=1, \ldots, n-1, \quad \text { and } \\
& h_{n}\left(u ; z_{1}, \ldots, z_{n}\right):=z_{n}^{d+1} \frac{\partial \widehat{\phi}_{u}}{\partial z_{n}}=z_{n} \frac{\partial f_{u}\left(z_{1}, \ldots, z_{n}\right)}{\partial z_{n}}-d \cdot f_{u}\left(z_{1}, \ldots, z_{n}\right) .
\end{aligned}
$$

Suppose that $w \in C$ satisfies $\rho(w) \in \mathscr{A} \cap \mathcal{U}_{N}$. Since $\tilde{q}_{1}(w), \ldots, \tilde{q}_{N}(w)$ are the critical points of $\widehat{\phi}_{\rho(w)}$, the definition of $h_{\imath}(u ; z)$ implies $h_{t}\left(\rho(w) ; \tilde{q}_{j}(w)\right)=0$ holds for $i=1, \ldots, n$ when $\tilde{q}_{j}(w) \in\left(\mathbf{A}^{n}\right)^{\prime}$. By continuity, we see the following:

$$
h_{\imath}\left(c ; \tilde{q}_{j}(\tilde{c})\right)=0 \text { for all } i=1, \ldots, n \text { and } j=1, \ldots, N
$$


Let $H_{i}(u)$ be the affine hypersurface in $\left(\mathbb{A}^{n}\right)^{\prime}$ defined by $h_{i}(u ; z)=0$. We put

$$
I(u):=H_{1}(u) \cap \ldots \cap H_{n}(u)
$$

By the definition of $h_{i}(u ; z)$, the set $I(u) \backslash\left(I(u) \cap H_{\infty}\right)$ coincides with the set of critical points of $\bar{\phi}_{u}$ contained in $\left(\mathbb{A}^{n}\right)^{\prime}$. Recall that $\tilde{q}_{1}(\tilde{c}), \ldots, \tilde{q}_{N}(\tilde{c}) \in\left(\mathbb{A}^{n}\right)^{\prime}$. Since $\Delta$ is small enough, the critical points $\tilde{q}_{1}(w), \ldots, \tilde{q}_{N}(w)$ of $\widehat{\phi}_{\rho(w)}$ remain in $\left(\mathbb{A}^{n}\right)^{\prime}$ for all $w \in \widetilde{\Delta} \backslash\{\tilde{c}\}$.

$$
I(\rho(w)) \backslash\left(I(\rho(w)) \cap H_{\infty}\right)=\left\{\tilde{q}_{1}(w), \ldots, \tilde{q}_{N}(w)\right\} \text { for all } w \in \tilde{\Delta} \backslash\{\tilde{c}\}
$$

These constructions and properties will be used in the proofs of Propositions 3.1 and 9.6.1.

\section{\$3. Boundaries of $\mathbb{F}_{u}$}

In this section, we always assume $u \in \mathcal{U}$.

Note that $0 \notin \mathrm{Cr}(u)$ by (2.1). We define a function $\widetilde{\varepsilon}: \mathcal{U} \rightarrow \mathbb{R}_{>0}$ by

$$
\widetilde{\varepsilon}(u):=\min \left\{|p|,|p|^{-1} ; p \in \operatorname{Cr}(u)\right\} .
$$

\section{Proposition 3.1. This function $\widetilde{\varepsilon}$ is continuons.}

Proof. Since $\operatorname{Cr}(u)=\iota_{u}^{-1}\left(\mathscr{L}_{u} \cap \mathscr{D}_{0}\right)$ by Corollary 2.1 , the critical values vary continuously as $u$ moves. Hence all we have to do is to delete the possibility that there might be a point $c \in \mathcal{U}$ such that, if $u$ approaches $c$, then a critical value of $\widehat{\phi}_{u}$ tends to $\infty$. Suppose that such a point $c$ exists in $U$. We choose a general affine line $\mathscr{A} \subset \Gamma^{\times}$passing through $c$, a small closed disk $\Delta$ $\subset \mathscr{A}$ with the center $c$, and a base point $b \in \partial \Delta$, and apply Constructions 2.1 and 2.2. The assumed property of the point $c$ implies that there exists at least one critical value among $\left\{\tilde{p}_{1}, \ldots, \tilde{p}_{N}\right\}$, say $\tilde{p}_{1}$, such that $\tilde{p}_{1}(\tilde{c})=\infty$. This implies that $\tilde{q}_{1}(\tilde{c}) \in H_{\infty}$. Combining this with (2.8), we see that the coordinates of the point $\tilde{q}_{1}(\tilde{c})$ are the solutions of

$$
\begin{aligned}
& z_{n}=f_{c}\left(z_{1}, \ldots, z_{n-1}, 0\right)=0, \quad \text { and } \\
& \frac{\partial f_{c}\left(z_{1}, \ldots, z_{n-1}, 0\right)}{\partial z_{i}}=0 \text { for } i=1, \ldots, n-1 .
\end{aligned}
$$

Since $f_{c}\left(z_{1}, \ldots, z_{n-1}, 0\right)=0$ defines the hypersurface $\bar{X}_{c} \cap H_{\infty}$ on $H_{\infty}$, the solution 
$\tilde{q}_{1}(\tilde{c})$ must be a singular point of $\bar{X}_{c} \cap H_{\infty}$. This contradicts the fact $c \in \mathcal{U}$.

Suppose that $\varepsilon: \mathcal{U} \rightarrow \mathbf{R}$ is a continuous function which satisfies

$$
0<\varepsilon(u)<\widetilde{\varepsilon}(u) \text { for all } u \in \mathcal{U} \text {, }
$$

whose existence is guaranteed by Proposition 3.1. We put

$$
B_{u}^{0}:=\left\{z \in \mathbb{C}^{\times} ; 0<|z| \leq \varepsilon(u)\right\}, \quad \text { and } \quad B_{u}^{\infty}:=\left\{z \in \mathbb{C}^{\times} ;|z|^{-1} \leq \varepsilon(u)\right\} \text {, }
$$

each of which is a punctured closed disk on $\mathbb{P}^{1}=\mathbb{C} \cup\{\infty\}$. We also put

$$
\partial_{0} E_{u}:=\phi_{u}^{-1}\left(B_{u}^{0}\right) \subset E_{u}, \quad \partial_{\infty} E_{u}:=\phi_{u}^{-1}\left(B_{u}^{\infty}\right) \subset E_{u},
$$

and

$$
\begin{aligned}
& \partial_{0} F_{u}:=\tilde{e}^{-1}\left(\partial_{0} E_{u}\right)=\phi_{u}^{-1}\left(e^{-1}\left(B_{u}^{0}\right)\right) \subset F_{u}, \\
& \partial_{\infty} F_{u}:=\tilde{e}^{-1}\left(\partial_{\infty} E_{u}\right)=\phi_{u}^{-1}\left(e^{-1}\left(B_{u}^{\infty}\right)\right) \subset F_{u} .
\end{aligned}
$$

(See (1.1) for the definition of $\tilde{e}$ and $\phi_{u}$.) We put

$$
C r(u):=e^{-1}(\operatorname{Cr}(u))
$$

Then the definition (1.1) of $\phi_{u}: F_{u} \rightarrow \mathbf{C}$ and Proposition 2.1 implies the following :

Proposition 3.2. The set of critical values of $\phi_{u}: F_{u} \rightarrow \mathbb{C}$ coincides with $\mathscr{C} r(u)$, and $\phi_{u}$ is locally trivial over $\mathbb{C} \backslash \mathscr{C} r(u)$.

By the definition (3.1) of $\widetilde{\boldsymbol{\varepsilon}}$ and the condition (3.2), there are no critical points of $\phi_{u}: F_{u} \rightarrow \mathbf{C}$ in $\partial_{0} F_{u}$ and in $\partial_{\infty} F_{u}$. Moreover, each of the subspaces

$$
\mathbf{C} \backslash e^{-1}\left(B_{u}^{0}\right) \subset \mathbf{C}, \quad \mathbf{C} \backslash e^{-1}\left(B_{u}^{\infty}\right) \subset \mathbf{C}, \quad \text { and } \quad \mathbf{C} \backslash\left(e^{-1}\left(B_{u}^{0}\right) \cup e^{-1}\left(B_{u}^{\infty}\right)\right) \subset \mathbf{C}
$$

is a strong deformation retract of $\mathbb{C}$. Hence, by Proposition 3.2, each of the subspaces

$$
F_{u} \backslash \partial_{0} F_{u} \subset F_{u}, \quad F_{u} \backslash \partial_{\infty} F_{u} \subset F_{u}, \quad \text { and } \quad F_{u} \backslash\left(\partial_{0} F_{u} \cup \partial_{\infty} F_{u}\right) \subset F_{u}
$$

is also a strong deformation retract of $F_{u}$. Therefore, we can call $\partial_{0} F_{u}$ and 
$\partial_{\infty} F_{u}$ the boundaries of $F_{u}$. In particular, since $\partial_{0} F_{u} \cap \partial_{\infty} F_{u}=\emptyset$, the intersection pairing

$$
\langle,\rangle: H_{n}\left(F_{u}, \partial_{\infty} F_{u}\right) \times H_{n}\left(F_{u}, \partial_{0} F_{u}\right) \rightarrow \mathbb{Z}
$$

between the relative homology groups is well defined.

It is obvious that each of the pairs $\left(F_{u}, \partial_{0} F_{u}\right)$ and $\left(F_{u}, \partial_{\infty} F_{u}\right)$ forms a local. ly trivial family over $U$ when $u$ varies. Moreover, the deck transformation $T_{u}$ : $F_{u} \rightarrow F_{u}$ induces automorphisms of $\partial_{0} F_{u}$ and $\partial_{\infty} F_{u}$. Hence $H_{n}\left(F_{u}, \partial_{0} F_{u}\right)$ and $H_{n}\left(F_{u}, \partial_{\infty} F_{u}\right)$ can be regarded as $\mathbb{Z}\left[q, q^{-1}\right]$-modules in the same way as $H_{n}\left(F_{u}\right)$. Therefore, each of $H_{n}\left(F_{u}, \partial_{0} F_{u}\right)$ and $H_{n}\left(F_{u}, \partial_{\infty} F_{u}\right)$ forms a locally constant system of $\mathbb{Z}\left[q, q^{-1}\right]$-modules over $\mathcal{U}$. We thus obtain natural monodromy representations

$$
\pi_{1}(U, b) \rightarrow \operatorname{Aut}_{\mathbb{Z}\left[q, q^{-1}\right]}\left(H_{n}\left(F_{b}, \partial_{0} F_{b}\right)\right) \text { and } \pi_{1}(U, b) \rightarrow \operatorname{Aut}_{\mathbb{Z}\left[q, q^{-1}\right]}\left(H_{n}\left(F_{b}, \partial_{\infty} F_{b}\right)\right)
$$

which are compatible with (1.2) via the natural homomorphisms $H_{n}\left(F_{b}\right) \rightarrow$ $H_{n}\left(F_{b}, \partial_{0} F_{b}\right)$ and $H_{n}\left(F_{b}\right) \rightarrow H_{n}\left(F_{b}, \partial_{\infty} F_{b}\right)$ of $\mathbb{Z}\left[q, q^{-1}\right]$-modules.

Remark 3.1. The homeomorphism types of all spaces $\left(E_{u}, \partial_{0} E_{u}\right),\left(E_{u}, \partial_{\infty} E_{u}\right)$, $\left(F_{u}, \partial_{0} F_{u}\right),\left(F_{u}, \partial_{\infty} F_{u}\right)$, and so on, or of the maps between them are independent of the choice of the function $\varepsilon$, provided that (3.2) is fulfilled. Therefore, we will not specify any particular choice of $\varepsilon$. Sometimes, however, we pick up a sufficiently small positive real number $r$, and use the function $\varepsilon:=\min \{\widetilde{\varepsilon} / 2, r\}$, so that $\varepsilon$ is a constant function on a given compact subset of $\mathcal{U}$.

\section{\$4. Vanishing Cycles ard Thimbles}

In this section, we fix notion and notation concerned with vanishing cycles for ordinary double points and associated thimbles. For the proofs of the facts stated in this section, we refer the reader to [8].

Let $S^{n-1}$ be an oriented $(n-1)$-sphere, and let $r \in\left[S^{n-1}, S^{n-1}\right]$ be the homotopy class of orientation-reversing self-homeomorphisms. Note that $r^{2} \in$ $\left[S^{n-1}, S^{n-1}\right]$ is the homotopy class of the identity. For a topological space $T$ and a homotopy class $f \in\left[S^{n-1}, T\right]$, we write by $-f \in\left[S^{n-1}, T\right]$ the homotopy class $f \circ r$. Note that, since $S^{n-1}$ is oriented, we have a natural map $\left[S^{n-1}, T\right]$ $\rightarrow H_{n-1}(T)$. 
We denote by $C S^{n-1}$ the cone over $S^{n-1}$; that is, the space obtained from $I$ $\times S^{n-1}$ by contracting $\{1\} \times S^{n-1}$ to a point, which is the vertex of the cone. We equip $C S^{n-1}$ with the orientation induced from that of the product space $I \times$ $S^{n-1}$. Hence we have

$$
\partial C S^{n-1}=-S^{n-1} \text {. }
$$

Therefore, for a pair $(T, S)$ of a topological space $T$ and its subspace $S$, there is a natural map $\left[\left(C S^{n-1}, S^{n-1}\right),(T, S)\right] \rightarrow H_{n}(T, S)$, which makes the following diagram anti-commutative :

$$
\begin{array}{ccc}
{\left[\left(C S^{n-1}, S^{n-1}\right),(T, S)\right]} & \rightarrow & H_{n}(T, S) \\
\downarrow \text { restriction } & & \downarrow \text { o } \\
{\left[S^{n-1}, S\right]} & \rightarrow & H_{n-1}(S)
\end{array}
$$

There is a unique class $\tilde{r} \in\left[\left(C S^{n-1}, S^{n-1}\right),\left(C S^{n-1}, S^{n-1}\right)\right]$ which is represented by an orientation-reversing self-homeomorphism. For $f \in\left[\left(C S^{n-1}\right.\right.$, $\left.\left.S^{n-1}\right),(T, S)\right]$, we write by $-f$ the homotopy class $f \circ \tilde{r}$.

Now we consider the following situation. Let $W$ be a non-singular connected complex manifold of dimension $n, Z$ a Riemann surface, and $g: W \rightarrow Z$ a surjective holomorphic map. For a point $z \in Z$, let $W_{z}$ denote the fiber $g^{-1}$ $(z)$. Suppose that the following conditions $(w z-1)-(w z-3)$ are satisfied. (wz-1) The map $g$ has only one critical point $q \in W$, which is non-degenerate. (wz-2) Moreover, $g$ is locally trivial over $Z \backslash\{p\}$, where $p=g(q)$.

Because of $(w z-1)$, there exist local analytic coordinates $\left(w_{1}, \ldots, w_{n}\right)$ on $W$ with the center $q$ and an analytic coordinate $t$ on $Z$ with the center $p$ such that $g$ is given by

$$
\left(w_{1}, \ldots, w_{n}\right) \mapsto t=w_{1}^{2}+\cdots+w_{n}^{2}
$$

locally around $q$. We choose a small positive real number $\epsilon$ and a positive real number $\eta$ which is small enough compared with $\epsilon$, and put

$$
B_{\epsilon}:=\left\{\left(w_{1}, \ldots, w_{n}\right) \in W ;\left|w_{1}\right|^{2}+\cdots+\left|w_{n}\right|^{2} \leq \epsilon\right\} \quad \text { and } \quad \Delta_{\eta}:=\{t \in Z ;|t| \leq \eta\}
$$

The third condition we impose is the following:

(wz-3) the restriction of $g$ to $\left(g^{-1}\left(\Delta_{\eta}\right) \backslash \operatorname{Int} B_{\epsilon}, g^{-1}\left(\Delta_{\eta}\right) \cap \partial B_{\epsilon}\right)$ is trivial over $\Delta$. 
Note that the conditions $\left(w z^{-1}\right)-\left(w z^{-3}\right)$ imply that $g^{-1}(p)$ is a strong deformation retract of $g^{-1}\left(\Delta_{\eta}\right)$.

The cases we are going to apply the facts explained in this section are, for example, as follows. Let $u$ be a point on $\boldsymbol{U}_{N}$, and $p \in \mathbb{C}$ a value in $\operatorname{Cr}(u)$. Then the situation

$$
Z=\mathbb{C}^{\times} \backslash(\operatorname{Cr}(u) \backslash\{p\}), \quad W=E_{u} \backslash \bigcup_{p^{\prime} \in \operatorname{Cr}(u) \backslash\{p\}} \phi_{u}^{-1}\left(p^{\prime}\right), \quad \text { and } g=\left.\phi_{u}\right|_{W}
$$

satisfies the conditions $(w z-1)-(w z-3)$ because of Propositions 2.1, 2.5 and the definition of $\mathcal{U}_{N}$. We will also consider the following situation. Let $u$ be as above, and let $\tilde{p} \in \mathbb{C}$ be a value in $\mathscr{C} r(u)$. Then the data

$$
Z=\mathbb{C} \backslash(\mathscr{C} r(u) \backslash\{\tilde{p}\}), \quad W=F_{u} \backslash \bigcup_{\tilde{p^{\prime}} \in \mathscr{C} r(u) \backslash\{\tilde{p}\}} \phi_{u}^{-1}\left(\tilde{p}^{\prime}\right), \quad \text { and } \quad g=\left.\phi_{u}\right|_{W}
$$

satisfy the conditions $(w z-1)-(w z-3)$ because $\phi_{u}$ is the pull-back of $\phi_{u}$ by the étale covering $e: \mathbb{C} \rightarrow \mathbb{C}^{\times}$.

Now we go back to the general situation.

Definition 4.1. Let $a$ be a point on $Z \backslash\{p\}$, and let $\mathscr{P}(a)$ be the space of all paths $\omega: I \rightarrow Z$ from $a$ to $p$ such that $p \notin \omega([0,1))$. We equip $\mathscr{P}(a)$ with the compact-open topology, and let $[\mathscr{P}(a)]$ denote the set of path-connected components of $\mathscr{P}(a)$. For $\omega \in \mathscr{P}(a)$, let $[\omega] \in[\mathscr{P}(a)]$ denote the pathconnected component containing $\omega$; that is, the homotopy class of paths in $\mathscr{P}(a)$ represented by $\omega$.

Proposition 4.1. For a point $a \in Z \backslash\{p\}$ and a homotopy class $[\omega] \in$ $[\mathscr{P}(a)]$, there exists a homotopy class $\sigma[\omega] \in\left[S^{n-1}, W_{a}\right]$, unique up to sign, which has the following properties. (i) Let $a^{\prime}$ be another point on $Z \backslash\{p\}$, and $\tau: I \rightarrow Z \backslash\{p\}$ a path from $a^{\prime}$ to $a$. Then we have

$$
\sigma[\omega \cdot \tau]= \pm[\tau]^{-1}(\sigma[\omega])
$$

where $[\tau]_{*}:\left[S^{n-1}, W_{a^{\prime}}\right] \rightarrow\left[S^{n-1}, W_{a}\right]$ is the bijective map induced from the triviality of $g: W \rightarrow Z$ over $\tau: I \rightarrow Z \backslash\{p\}$.: (ii) Suppose that $a \in \Delta_{\eta} \backslash\{p\}$ and $\omega(I)$ $\subset \Delta_{n}$. Then $\sigma[\omega] \in\left[S^{n-1}, W_{a}\right]$ is represented by a continuous map 


$$
S^{n-1} \rightarrow B_{\epsilon} \cap W_{a} \hookrightarrow W_{a}
$$

such that the map $S^{n-1} \rightarrow B_{\epsilon} \cap W_{a}$ induces homotopy equivalence.

Sketch of Proof. Let $a$ be a point on $\Delta_{\eta} \backslash\{p\}$. The fact that $B_{\epsilon} \cap W_{a}$ is homotopically equivalent to $S^{n-1}$ follows from (4.2) (cf. [8; p. 37]). Hence $\sigma[\omega] \in\left[S^{n-1}, W_{a}\right]$ is uniquely determined, up to sign, by the property (ii), when $\omega$ is a path in $\Delta_{n}$. For an arbitrary $a \in Z \backslash\{p\}$ and an arbitrary $\omega \in$ $\mathscr{P}(a)$, there exists $\lambda \in(0,1)$ such that $\omega([\lambda, 1]) \subset \Delta_{\eta}$. We decompose $\omega$ into $\omega_{2} \cdot \omega_{1}$ at $\lambda$ : that is, $\omega_{1}(t)=\omega(\lambda t)$ and $\omega_{2}(t)=\omega(\lambda+t(1-\lambda))$. By the above argument, we have $\sigma\left[\omega_{2}\right] \in\left[S^{n-1}, W_{\omega(\lambda)}\right]$. The class $\sigma[\omega] \in\left[S^{n-1}\right.$, $\left.W_{a}\right]$ is derived from $\sigma\left[\omega_{2}\right]$ via the bijective map between $\left[S^{n-1}, W_{a}\right]$ and $\left[S^{n-1}\right.$, $\left.W_{\omega(\lambda)}\right]$ induced by the triviality of $g$ over $\omega_{1}$, using property (i).

Definition 4.2. We call the class $\sigma[\omega] \in\left[S^{n-1}, W_{a}\right]$ the vanishing cycle for $[\omega]$. Let $\bar{\sigma}[\omega] \in H_{n-1}\left(W_{a}\right)$ denote the corresponding homology class.

Remark 4.1. Traditionally, the homology class $\bar{\sigma}[\omega]$ has been called the vanishing cycle for $[\omega]$.

Remark 4.2. There are usually two vanishing cycles $\sigma[\omega]$ and $-\sigma[\omega]=$ $\sigma[\omega] \circ r$ for a given $[\omega]$.

Let $W \times{ }_{Z} I_{\omega}$ be the pull-back of $g: W \rightarrow Z$ by $\omega: I \rightarrow Z$, where $\omega \in \mathscr{P}(a)$. Then the inclusion $W_{b} \hookrightarrow W \times{ }_{z} I_{\omega}$ induces homotopy equivalence because of (wz-1) - (wz-3). Combining the embedding $W_{a} \hookrightarrow W \times{ }_{z} I_{\omega}$ with the homotopy inverse $W \times_{z} I_{\omega} \rightarrow W_{p}$ of the inclusion, we get a contraction map

$$
C_{\omega}: W_{a} \rightarrow W_{p}
$$

along $\omega$. Let $\zeta: I \rightarrow Z \backslash\{p\}$ be a loop with the base point $a$ as follows : $\zeta$ goes along $\omega$ from $a$ to a point $p^{\prime}:=\omega(1-\lambda) \in \Delta_{\eta}$, where $\lambda$ is a positive real number small enough, draws a circle in the punctured disk $\Delta_{\eta} \backslash\{p\}$ from $p^{\prime}$ to $p^{\prime}$ in the counter-clockwise direction, and goes back to $a$ along $\omega^{-1}$.
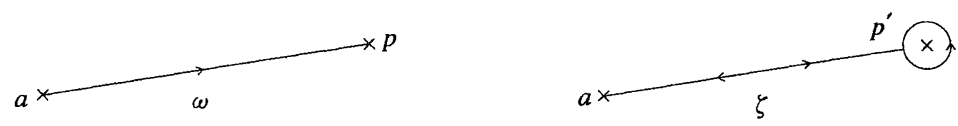

Figure 1 
Then we have the monodromy action

$$
[\zeta]_{*}: H_{n-1}\left(W_{a}\right) \rightarrow H_{n-1}\left(W_{a}\right)
$$

induced by $[\zeta] \in \pi_{1}(Z \backslash\{p\}, a)$. The classical theory of Lefschetz states the following :

Theorem $\mathbb{L} 1$. (1) The kernel of $C_{\omega *}: H_{n-1}\left(W_{a}\right) \rightarrow H_{n-1}\left(W_{p}\right)$ is generated by the homology class $\bar{\sigma}[\omega]$ of a vanishing cycle for $[\omega]$. (2) The image of the endomorphism Id $-[\zeta]_{*}$ of $H_{n-1}\left(W_{a}\right)$ is contained in the kernel of $C_{\omega *}$.

Now we describe the notion of thimbles. Let

$$
\rho: C S^{n-1} \rightarrow I
$$

be the natural projection induced from the first projection $I \times S^{n-1} \rightarrow I$.

Proposition 4.2. Suppose that $a \in Z \backslash\{p\}$ and $\omega \in \mathscr{P}(a)$ are given. Suppose also that the sign of the vanishing cycle $\sigma[\omega]$ is specified. Then there exists a unique homotopy class

$$
\theta([\omega], \sigma[\omega]) \in\left[\left(C S^{n-1}, S^{n-1}\right),\left(W, W_{a}\right)\right]
$$

with the following properties. (i) The image of $\theta([\omega], \sigma[\omega])$ by the natural map

$$
\left[\left(C S^{n-1}, S^{n-1}\right),\left(W, W_{a}\right)\right] \rightarrow\left[S^{n-1}, W_{a}\right]
$$

is $\sigma[\omega]$. (ii) The homotopy class $\theta([\omega], \sigma[\omega])$ is represented by a continuous map $T: C S^{n-1} \rightarrow W$ which makes the following diagram commutative

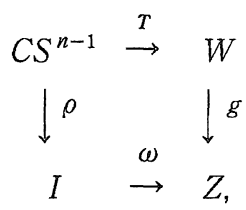

and which maps the vertex of the cone $C S^{n-1}$ to the critical point $q$.

Sketch of Proof. Suppose that $\sigma[\omega] \in\left[S^{n-1}, W_{a}\right]$ is represented by $s_{0}$ : $S^{n-1} \rightarrow W_{a}$. Then $s_{0}$ deforms continuiously to $s_{t}: S^{n-1} \rightarrow W_{\omega(t)}$ for $t \in[0,1]$. We see from the property (ii) of the vanishing cycle that $s_{1}$ is homotopically equivalent to the constant map $S^{n-1} \rightarrow\{q\} \hookrightarrow W_{p}$, because $B_{\epsilon} \cap W_{p}$ is contracti- 
ble by (4.2). Therefore, by changing the deformation $s_{t}$ homotopically, we may assume that $s_{1}$ is the constant map through $\{q\}$. The continuous map $T$ is constructed by putting these $s_{t}$ together.

Definition 4.3. We call the homotopy class $\theta([\omega], \sigma[\omega])$ the thimble for $[\omega]$ starting from $\sigma[\omega]$. When the orientation does not need to be specified, we write this thimble simply by $\theta([\omega])$. (Note that $\theta([\omega],-\sigma[\omega])=-\theta([\omega]$, $\sigma[\omega])$.) We denote its homology class by $\bar{\theta}([\omega], \sigma[\omega]) \in H_{n}\left(W, W_{a}\right)$.

Definition 4.4 Suppose that $\omega^{\prime} \in \mathscr{P}(a)$ is a path representing a homotopy class $[\omega] \in[\mathscr{P}(a)]$. We say that a continunus map $T: C S^{n-1} \rightarrow W$ represents the thimble $\theta([\omega], \sigma[\omega])$ over the path $\omega^{\prime}$, if the diagram

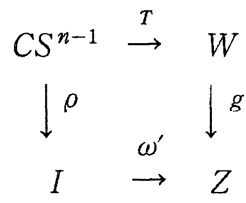

is commutative (in particular, $T\left(\{0\} \times S^{n-1}\right)$ is contained in $W_{a}$ ) and if $T$ represents $\theta([\omega], \sigma[\omega])$ in $\left[\left(C S^{n-1}, S^{n-1}\right),\left(W, W_{a}\right)\right]$.

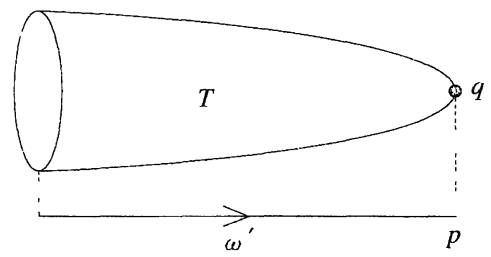

Figure 2

It is obvious that, for any $\omega^{\prime} \in[\omega]$, there exists a continuous map $T: C S^{n-1} \rightarrow$ $W$ which represents the thimble $\theta([\omega], \sigma[\omega])$ over $\omega^{\prime}$.

Definition 4.5. Let $\xi: I \rightarrow Z$ be a sub-path of $\omega$; that is, there is a continuous increasing map $i: I \rightarrow I$ such that $\xi=\omega \circ i$. Let $T: C S^{n-1} \rightarrow W$ be a continuous map representing the thimble $\theta([\omega], \sigma[\omega])$ over $\omega$. The restriction $T\}_{\xi}$ of $T$ to $\xi$ is the composite of $\tilde{i}:\left(C S^{n-1}\right) \times_{I} I \rightarrow C S^{n-1}$ and $T$, where $\tilde{i}$ is the pull-back of $i$ by $\rho: C S^{n-1} \rightarrow I$. If $i(1)<1$, then $\left.T\right|_{\xi}$ is a map from $I \times S^{n-1}$ to $W$. If $i(1)=1$, then $\left.T\right|_{\xi}$ is a continuous map from $C S^{n-1}$ to $W$, which repre- 
sents the thimble $\theta([\xi])$ over the path $\xi \in \mathscr{P}(\xi(0))$.
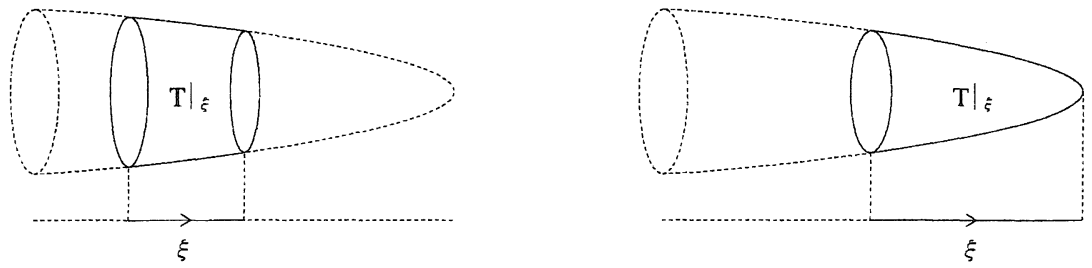

Figure 3

Now we choose two points $a$ and $a^{\prime}$ in $\Delta_{\eta} \backslash\{p\}$ such that the radius of the disk $\Delta_{\eta}$ passing through $a$ and the radius passing through $a^{\prime}$ are distinct. Let $\omega$ and $\omega^{\prime}$ be the paths from $a$ and $a^{\prime}$, respectively, to the center $p$ along the radius of the disk $\Delta_{\eta}$. Let $c_{+}$and $\iota_{-}$be the paths in $\Delta_{\eta} \backslash\{p\}$ from $a$ to $a^{\prime}$ described as follows : the path $\iota_{+}$(resp. $\iota_{-}$) starts from $a$, goes to a point on the boundary $\partial \Delta_{\eta}$ along the radius, draws an arc on $\partial \Delta_{\eta}$ in the counter-clockwise direction (resp. in the clockwise direction) to the end point of the radius passing through $a^{\prime}$, and then goes to $a^{\prime}$ along this radius.

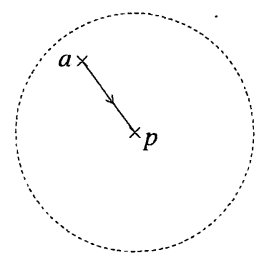

$\omega$
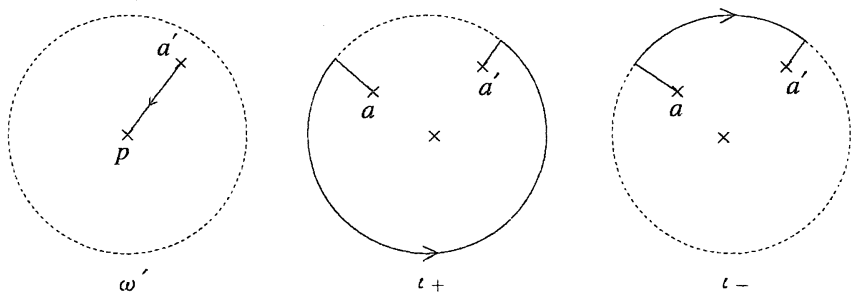

Figure 4

Suppose that a vanishing cycle $\sigma[\omega] \in\left[S^{n-1}, W_{a}\right]$ for $[\omega]$ is chosen from among the two possibilities. We put

$$
\left.\sigma_{+}\left[\omega^{\prime}\right]:=\ell_{+}\right]_{*}(\sigma[\omega]) \text {, and } \sigma_{-}\left[\omega^{\prime}\right]:=\left[\iota_{-}\right]_{*}(\sigma[\omega]) \text {, }
$$

both of which are vanishing cycles for $\left[\omega^{\prime}\right]$, because $\left[\omega \cdot \iota_{+}^{-1}\right]=\left[\omega \cdot \iota_{-}^{-1}\right]=$ $\left[\omega^{\prime}\right]$ in $\left[\mathscr{P}\left(a^{\prime}\right)\right]$. Then we have

$$
\sigma_{+}\left[\omega^{\prime}\right]=(-1)^{n} \sigma_{-}\left[\omega^{\prime}\right] \text { in }\left[S^{n-1}, W_{a^{\prime}}\right]
$$


Let $T, T_{+}$and $T_{-}$be continuous maps from $C S^{n-1}$ to $W$ which represent the thimbles $\theta([\omega], \sigma[\omega]), \theta\left(\left[\omega^{\prime}\right], \sigma_{+}\left[\omega^{\prime}\right]\right)$ and $\theta\left(\left[\omega^{\prime}\right], \sigma_{-}\left[\omega^{\prime}\right]\right)$, respectively, over $\omega, \omega^{\prime}$ and $\omega^{\prime}$, respectively. With the orientation of $C S^{n-1}$, we can consider these maps as $n$-chains in $W$.

Lemma 4.1. We can choose the maps $T, T_{+}$and $T_{-}$in such a way that the $n$-chains $T$ and $T_{+}$(resp. $T$ and $T_{-}$) intersect at only one point $q$ transversely with the intersection number $(-1)^{n(n-1) / 2}$ (resp. $\left.(-1)^{n(n+1) / 2}\right)$.

Proof. This lemma can be checked by direct calculation using the explicit form (4.2) of $g$ near the critical point $q$.

\section{§5. Structures of $\boldsymbol{H}_{n-1}\left(\boldsymbol{X}_{u}\right), \boldsymbol{H}_{n}\left(\boldsymbol{E}_{u}\right)$ and $\boldsymbol{H}_{n}\left(\boldsymbol{E}_{u}, \partial_{0} \boldsymbol{E}_{u}\right)$}

In this section, we always assume that $u \in \mathcal{U}$. We define two points

$$
a_{u}^{0}:=\varepsilon(u), \quad \text { and } \quad a_{u}^{\infty}:=1 / \varepsilon(u)
$$

on $\mathbf{C}^{\times}$, and consider the fibers

$$
X_{u}^{0}:=\phi_{u}^{-1}\left(a_{u}^{0}\right), \quad \text { and } \quad X_{u}^{\infty}:=\phi_{u}^{-1}\left(a_{u}^{\infty}\right)
$$

By the property (3.2) of $\varepsilon: \mathcal{U} \rightarrow \mathbb{R}_{>0}$, there are no critical values of $\widehat{\phi}_{\boldsymbol{u}}$ on the interval $[0, \varepsilon(u)] \subset \mathbb{R}$. Hence, by Proposition 2.1, there is a diffeomorphism, unique up to homotopy,

$$
X_{u}=\bar{\phi}_{u}^{-1}(0) \cong X_{u}^{0}
$$

which is induced by the path from 0 to $\varepsilon(u)$ along $\mathbf{R}$. It is obvious that each of the families $\left\{X_{u}^{0} ; u \in \mathcal{U}\right\}$ and $\left\{X_{u}^{\infty} ; u \in \mathcal{U}\right\}$ is locally trivial over $\mathcal{U}$, and hence $\pi_{1}(U, b)$ acts on $H_{n-1}\left(X_{b}^{0}\right)$ and on $H_{n-1}\left(X_{b}^{\infty}\right)$. The lemma below follows immediately from the definition of $\varepsilon$.

Lemma 5.1. The isomorphism $H_{n-1}\left(X_{b}\right) \cong H_{n-1}\left(X_{b}^{0}\right)$ induced by (5.1) is $\pi_{1}(u, b)$-equivariant.

Since $a_{u}^{\infty} \notin \operatorname{Cr}(u)$, Proposition 2.1 implies that $X_{u}$ and $X_{u}^{\infty}$ are also diffeomorphic. However, the homotopy class of this diffeomorphism is not uniquely determined, and we cannot expect that there exists a $\pi_{1}(\mathcal{U}, b)$-equivariant iso- 
morphism $H_{n-1}\left(X_{b}\right) \cong H_{n-1}\left(X_{b}^{\infty}\right)$ by any means.

Note the following:

Theorem $\mathbb{L}_{2}$ (Lefschetz Hyperplane Section Theorem)。The homology groups $H_{i}\left(X_{u}\right) \cong H_{i}\left(X_{u}^{0}\right) \cong H_{i}\left(X_{u}^{\infty}\right)$ are zero for $i>n-1$.

Proof. See, for example, [12; Theorem 7.1].

Definition $\mathscr{D}_{0} \mathbb{1}$. For a point $a \in \mathbb{C}^{\times} \backslash \operatorname{Cr}(u)$ and $p \in \operatorname{Cr}(u)$, let $\mathscr{P}_{u}(a, p)$ denote the space of all paths $\omega: I \rightarrow \mathbb{C}^{\times}$which satisfy the following: (i) $\omega(0)=$ $a, \omega(1)=p$, and (ii) $\omega([0,1)) \cap \operatorname{Cr}(u)=\emptyset$. We equip $\mathscr{P}_{u}(a, p)$ with the compact-open topology. Let $\left[\mathscr{P}_{u}(a, p)\right]$ denote the set of path-connected components of $\mathscr{P}_{u}(a, p)$. For $\omega \in \mathscr{P}_{u}(a, p)$, let $[\omega] \in\left[\mathscr{P}_{u}(a, p)\right]$ denote the path-connected component containing $\omega$; that is, $[\omega]$ denotes the homotopy class of paths in $\mathscr{P}_{u}(a, p)$ represented by $\omega$.

Suppose that $u \in U_{N}$. Then $\operatorname{Cr}(u)$ consists of distinct $N$ values $\left\{p_{1}, \ldots, p_{N}\right\}$.

Definition 5.2. Suppose that $a \in \mathbb{C}^{\times} \backslash \mathrm{Cr}(u)$ is given. A set of paths $\left\{\xi_{1}, \ldots, \xi_{N}\right\}$, where $\xi_{i} \in \mathscr{P}_{u}\left(a, p_{i}\right)$, is called a regular system of paths from a if the following are satisfied: (i) each $\xi_{\imath}: I \rightarrow \mathbb{C}^{\times}$is injective, and (ii) $\xi_{\imath}(I) \cap \xi_{1}(I)=$ $\{a\}$ if $i \neq j$.

It is obvious that there always exists a regular system of paths for every $u \in$ $\mathcal{U}_{N}$ and every $a \in \mathbb{C}^{\times} \backslash \operatorname{Cr}(u)$.

Since $u \in U_{N}$, the morphism $\phi_{u}$ has only one critical point $q_{i}$ over each $p_{i}$. Moreover, these critical points are all non-degenerate. Therefore, if we are given a regular system $\left\{\xi_{1}, \ldots, \xi_{N}\right\}$ of paths from $a$, we obtain vanishing cycles $\pm \sigma\left[\xi_{l}\right] \in\left[S^{n-1}, \phi_{u}^{-1}(a)\right]$ for each $\left[\xi_{l}\right]$, and the associated thimbles

$$
\pm \theta\left(\left[\xi_{l}\right], \sigma\left[\xi_{l}\right]\right) \in\left[\left(C S^{n-1}, S^{n-1}\right),\left(E_{u}, \phi_{u}^{-1}(a)\right)\right]
$$

for each $\left[\xi_{l}\right]$.

Proposition 5.1. Suppose that $u \in U_{N}$.

(0) Suppose that $\left\{\xi_{1}^{0}, \ldots, \xi_{N}^{0}\right\}$ is a regular system of paths from $a_{u}^{0}$. We choose a vanishing cycle $\sigma\left[\xi_{i}^{0}\right] \in\left[S^{n-1}, X_{u}^{0}\right]$ for each $\left[\xi_{i}^{0}\right]$ from among the two possibilities. Then the homology classes $\bar{\sigma}\left[\xi_{1}^{0}\right], \ldots ; \bar{\sigma}\left[\xi_{N}^{0}\right]$ form a set of basis for the free $\mathbb{Z}$-module $H_{n-1}\left(X_{u}^{0}\right)$.

( $\infty)$ Suppose that $\left\{\xi_{1}^{\infty}, \ldots, \xi_{N}^{\infty}\right\}$ is a regular system of paths from $a_{u}^{\infty}$. We choose 
a vanishing cycle $\sigma\left[\xi_{i}^{\infty}\right] \in\left[S^{n-1}, X_{u}^{\infty}\right]$ for each $\left[\xi_{i}^{\infty}\right]$ from among the possibilities. Then the homology classes $\bar{\sigma}\left[\xi_{1}^{\infty}\right], \ldots, \bar{\sigma}\left[\xi_{N}^{\infty}\right]$ form a set of basis for the free $\mathbf{Z}$-module $H_{n-1}\left(X_{u}^{\infty}\right)$.

Proof. Since these two assertions can be proved in completely parallel ways, we prove only the assertion (0).

Let $\Delta_{i} \subset \mathbf{C}^{\times}$be a small closed disk with the center $p_{i}$. Since $\left\{\xi_{1}^{0}, \ldots, \xi_{N}^{0}\right\}$ is a regular system of paths, the union $\cup_{i=1}^{N}\left(\xi_{i}^{0}(I) \cup \Delta_{i}\right)$ is a strong deformation retract of $\mathbf{C}$, and it contains $\mathrm{Cr}(u)$ in its interior. By Proposition 2.1, the space

$$
A:=\widehat{\phi}_{u}^{-1}\left(\bigcup_{i=1}^{N}\left(\xi_{i}^{0}(I) \cup \Delta_{\imath}\right)\right)
$$

is also a strong deformation retract of $\mathbf{A}^{n}$. Hence $A$ is contractible. We decompose $A$ into the union of the two parts

$$
A_{1}:=\widehat{\phi}_{u}^{-1}\left(\bigcup_{i=1}^{N} \xi_{i}^{0}([0,1 / 2])\right), \quad \text { and } \quad A_{2}:=\widehat{\phi}_{u}^{-1}\left(\bigcup_{i=1}^{N}\left(\xi_{i}^{0}([1 / 2,1]) \cup \Delta_{i}\right)\right)
$$

By applying the Mayer-Vietoris sequence to this decomposition of the contractible space $A$, we obtain an isomorphism

$$
H_{n-1}\left(A_{1} \cap A_{2}\right) \stackrel{\sim}{\rightarrow} H_{n-1}\left(A_{1}\right) \oplus H_{n-1}\left(A_{2}\right)
$$

induced by the inclusions. Using Propositions 2.1 and 2.5 (2), we have canonical homotopy equivalences

$$
\begin{aligned}
A_{1} & \sim X_{u}^{0}, \\
A_{2} & \sim \coprod_{i=1}^{N} \phi_{u}^{-1}\left(\Delta_{i}\right) \sim \coprod_{i=1}^{N} \phi_{u}^{-1}\left(p_{i}\right), \quad \text { and } \\
A_{1} \cap A_{2} & \left.\sim \amalg^{N} X_{u}^{0} \quad \text { (the disjoint union of } N \text { copies of } X_{u}^{0}\right),
\end{aligned}
$$

through which the isomorphism (5.3) is written as follows :

$$
s \oplus\left(c_{1} \oplus \cdots \oplus c_{N}\right): \bigoplus_{\imath=1}^{N} H_{n-1}\left(X_{u}^{0}\right) \stackrel{\sim}{\rightarrow} H_{n-1}\left(X_{u}^{0}\right) \oplus \bigoplus_{i=1}^{N} H_{n-1}\left(\phi_{u}^{-1}\left(p_{\imath}\right)\right)
$$

where $s: \oplus_{i=1}^{N} H_{n-1}\left(X_{u}^{0}\right) \rightarrow H_{n-1}\left(X_{u}^{0}\right)$ is the summation $\left(x_{1}, \ldots, x_{N}\right) \mapsto x_{1}+\cdots+$ 
$x_{N}$, and $c_{i}: H_{n-1}\left(X_{u}^{0}\right) \rightarrow H_{n-1}\left(\phi_{u}^{-1}\left(p_{i}\right)\right)$ is the homomorphism induced by the contraction map $X_{u}^{0} \rightarrow \phi_{u}^{-1}\left(p_{i}\right)$ along $\xi_{i}^{0}$. Thus we get an isomorphism

$$
H_{n-1}\left(X_{u}^{0}\right) \cong \bigoplus_{i=1}^{N} \operatorname{Ker} c_{i}
$$

By Theorem L1, the kernel of $c_{i}$ is generated by the homology class $\vec{\sigma}\left[\xi_{i}^{0}\right]$ of a vanishing cycle for $\left[\xi_{i}^{0}\right]$. Hence all we have to do now is to show that the $\mathbb{Z}$-module $H_{n-1}\left(X_{u}^{0}\right)$ is torsion free of rank $N$; that is,

$$
b_{n-1}\left(X_{u}^{0}\right)=b_{n-1}\left(X_{u}\right)=N=(d-1)^{n},
$$

where $b_{n-1}$ denotes the $(n-1)$-st Betti number. This is a well-known formula.

Next we shall investigate $H_{n}\left(E_{u}\right)$ and $H_{n}\left(E_{u}, \partial_{0} E_{u}\right)$.

\section{Proposition 5.2. Suppose that $u \in \mathcal{U}$.}

(1) There exists an isomorphism between $H_{n-1}\left(X_{u}^{0}\right)$ and $H_{n}\left(\partial_{0} E_{u}\right)$.

(2) The inclusion $\partial_{0} E_{u} \hookrightarrow E_{u}$ induces an isomorphism $H_{n}\left(\partial_{0} E_{u}\right) \stackrel{\widetilde{\rightarrow}}{\rightarrow} H_{n}\left(E_{u}\right)$.

(3) The natural homomorphism $H_{n}\left(E_{u}\right) \rightarrow H_{\mathrm{n}}\left(E_{u}, \partial_{0} E_{u}\right)$ is a zero map.

(4) The boundary homomorphism $H_{n}\left(E_{u}, \partial_{0} E_{u}\right) \rightarrow H_{n-1}\left(\partial_{0} E_{u}\right)$ is an injection.

(5) The inclusion $X_{u}^{0} \hookrightarrow \partial_{0} E_{u}$ induces an injection $H_{n-1}\left(X_{u}^{0}\right) \hookrightarrow H_{n-1}\left(\partial_{0} E_{u}\right)$.

(6) There exists an isomorphism between $H_{n}\left(E_{u}, \partial_{0} E_{u}\right)$ and $H_{n-1}\left(X_{u}^{0}\right)$.

(7) Moreover, when $u=b$, all the homomophisms above between the homology groups are $\pi_{1}(U, b)$-equivariant.

Proof. Since the homomorphisms in (2) - (5) are defined by natural topological operations, they are obviously $\pi_{1}(\mathcal{U})$-equivariant. The fact that the isomorphisms in (1) and (6) are $\pi_{1}(U)$-equivariant can be seen from the construction below.

Let $\Delta_{\varepsilon(u)}(0) \subset \mathbb{C}$ be the closed disk of radius $\varepsilon(u)$ with the center 0 . We have

$$
B_{u}^{0}=\Delta_{\varepsilon(u)}(0) \backslash\{0\}
$$

Since there are no critical values of $\bar{\phi}: \mathbb{A}^{n} \rightarrow \mathbb{C}$ on $\Delta_{\varepsilon(u)}(0)$, Proposition $2.1 \mathrm{im}$ plies that there is a diffeomorphism

$$
\widehat{\phi}_{u}^{-1}\left(\Delta_{\varepsilon(u)}(0)\right) \cong \Delta_{\varepsilon(u)}(0) \times X_{u}^{0}
$$


over $\Delta_{\varepsilon(u)}(0)$ which induces the identity on $X_{u}^{0}$. By restricting it, we obtain a diffeomorphism

$$
\partial_{0} E_{u}=\phi_{u}^{-1}\left(B_{u}^{0}\right) \cong B_{u}^{0} \times X_{u}^{0}
$$

over $B_{u}^{0}$. Each of these diffeomorphisms is unique up to homotopy. Using Theorem L2 and the Künneth formula, we obtain a canonical isomorphism $H_{n}\left(\partial_{0} E_{u}\right) \cong H_{n-1}\left(X_{u}^{0}\right)$ and hence (1) is proved. The Künneth formula and (5.6) also imply that there is a canonical decomposition

$$
H_{n-1}\left(\partial_{0} E_{u}\right) \cong H_{n-1}\left(X_{u}^{0}\right) \oplus H_{n-2}\left(X_{u}^{0}\right)
$$

into a direct sum. The inclusion $H_{n-1}\left(X_{u}^{0}\right) \hookrightarrow H_{n-1}\left(\partial_{0} E_{n}\right)$ of the first factor is induced from the inclusion $X_{u}^{0} \hookrightarrow \partial_{0} E_{u}$. Thus (5) is proved. Using the excision property of homology groups and the diffeomorphism (5.5), we get

$$
\begin{aligned}
H_{n}\left(E_{u}, \partial_{0} E_{u}\right) \cong & H_{n}\left(\mathbb{A}^{n}, \widehat{\phi}_{u}^{-1}\left(\Delta_{\varepsilon(u)}(0)\right)\right) \\
& \cong H_{n-1}\left(\widehat{\phi}_{u}^{-1}\left(\Delta_{\varepsilon(u)}(0)\right)\right) \cong H_{n-1}\left(X_{u}^{0}\right) .
\end{aligned}
$$

Hence (6) is proved. We can easily see that this isomorphism coincides with the composite of the boundary map $H_{n}\left(E_{u}, \partial_{0} E_{u}\right) \rightarrow H_{n-1}\left(\partial_{0} E_{u}\right)$ and the projection $H_{n-1}\left(\partial_{0} E_{u}\right) \rightarrow H_{n-1}\left(X_{u}^{0}\right)$ onto the first factor in (5.7). Hence (4) is proved. The assertion (3) is a consequence of (2) and (4). Therefore only (2) remains to be proved.

It is enough to prove (2) when $u$ is a point of $U_{N}$, because each of $H_{n}\left(\partial_{0} E_{u}\right)$ and $H_{n}\left(E_{u}\right)$ forms a locally constant system over $u$ when $u$ varies. Let $\Delta_{\imath} \subset$ $\mathbb{C}^{\times}$be a small closed disk with the center $p_{i}$. We can take a regular system $\left\{\xi_{1}^{0}, \ldots, \xi_{N}^{0}\right\}$ of paths from $a_{u}^{0}$ in such a way that

$$
\xi_{i}^{0}(I) \cap \Delta_{\varepsilon(u)}(0)=\left\{a_{u}^{0}\right\} \quad \text { for } \quad i=1, \ldots, N
$$

Then the space

$$
B_{u}^{0} \cup \bigcup_{i=1}^{N}\left(\xi_{i}^{0}(I) \cup \Delta_{i}\right) \subset \mathbf{C}^{\times}
$$

is a strong deformation retract of $\mathbf{C}^{\times}$, and it contains $\operatorname{Cr}(u)$ in its interior. Hence the space 


$$
A^{\times}:=\phi_{u}^{-1}\left(B_{u}^{0} \cup \bigcup_{\imath=1}^{N}\left(\xi_{\imath}^{0}(I) \cup \Delta_{\imath}\right)\right)
$$

is also a strong deformation retract of $E_{u}$ by Proposition 2.1. Thus $H_{n}\left(E_{u}\right)$ is canonically isomorphic to $H_{n}\left(A^{\times}\right)$. We decompose $A^{\times}$into the union of $A$ defined by (5.2) and $\partial_{0} E_{u}=\phi_{u}^{-1}\left(B_{u}^{0}\right)$. Because of (5.9), we have $A \cap \partial_{0} E_{u}=X_{u}^{0}$. Recall that $A$ is contractible. Hence the Mayer-Vietoris sequence for this decomposition is written as follows :

$$
\begin{gathered}
\rightarrow H_{n}\left(X_{u}^{0}\right) \rightarrow H_{n}\left(\partial_{0} E_{u}\right) \rightarrow H_{n}\left(A^{\times}\right) \\
\rightarrow H_{n-1}\left(X_{u}^{0}\right) \rightarrow H_{n-1}\left(\partial_{0} E_{u}\right) \rightarrow \cdots
\end{gathered}
$$

Because of the injectivity of $H_{n-1}\left(X_{u}^{0}\right) \rightarrow H_{n-1}\left(\partial_{0} E_{u}\right)$ by $(5)$ and of $H_{n}\left(X_{u}^{0}\right)=0$ by Theorem L2, we see that inclusion $\partial_{0} E_{u} \hookrightarrow A^{\times}$induces an isomorphism between $H_{n}\left(\partial_{0} E_{u}\right)$ and $H_{n}\left(A^{\times}\right) \cong H_{n}\left(E_{u}\right)$.

As in Proposition 5.1, we will describe explicitly a set of basis for the free $\mathbb{Z}$-module $H_{n}\left(E_{u}, \partial_{0} E_{u}\right)$ when $u \in \mathcal{U}_{N}$.

Proposition 5.3. Suppose that $u \in U_{N}$. Let $\left\{\xi_{1}^{0}, \ldots, \xi_{N}^{0}\right\}$ be a regular system of paths from $a_{u}^{0}$. Let $\sigma\left[\xi_{i}^{0}\right] \in\left[S^{n-1}, X_{u}^{0}\right]$ be a vanishing cycle for $\left[\xi_{i}^{0}\right]$, and let

$$
\theta\left(\left[\xi_{\imath}^{0}\right], \sigma\left[\xi_{1}^{0}\right]\right) \in\left[\left(C S^{n-1}, \mathrm{~S}^{n-1}\right),\left(E_{u}, X_{u}^{0}\right)\right]
$$

be the thimble for $\left[\xi_{i}^{0}\right]$ starting from $\sigma\left[\xi_{i}^{0}\right]$. Then the homology classes $\bar{\theta}\left(\left[\xi_{1}^{0}\right]\right.$, $\left.\sigma\left[\xi_{1}^{0}\right]\right), \ldots, \bar{\theta}\left(\left[\xi_{N}^{0}\right], \sigma\left[\xi_{N}^{0}\right]\right)$ form a set of basis for $H_{n}\left(E_{u}, \partial_{0} E_{u}\right)$.

Proof. Note that, by the isomorphism from $H_{n}\left(E_{u}, \partial_{0} E_{u}\right)$ to $H_{n-1}\left(X_{u}^{0}\right)$ given in Proposition 5.2(6) or (5.8), the homology class $\bar{\theta}\left(\left[\xi_{i}^{0}\right], \sigma\left[\xi_{i}^{0}\right]\right)$ is mapped to $-\bar{\sigma}\left[\xi_{i}^{0}\right]$ because of the anti-commutativity of (4.1). Hence the assertion follows from Proposition 5.1.

Now we fix a base point $b \in \mathcal{U}$. We shall review the classical theory of Lefschetz about monodromy representations, and study the structure of $H_{n-1}$ $\left(X_{b}^{0}\right)$ as a $\pi_{1}(U, b)$-module. Again, we refer the reader to [8] for the proof.

Let $\bar{X}_{b}^{0} \subset \mathbb{P}^{n}$ be the projective compactification of the affine hypersurface $X_{b}^{0} \subset \mathbb{A}^{n}$. Taking Remark 2.1 into account, we see that $\bar{X}_{b}^{0}$ is non-singular from Lemma 2.1 and the definition of $a_{b}^{0}$. Moreover, the intersection $H_{\infty} \cap$ 
$\bar{X}_{b}^{0}$ coincides with $Y_{b}:=H_{\infty} \cap \bar{X}_{b}$ from (2.2). There is a canonical isomorphism

$$
H_{n-1}\left(X_{b}^{0}\right) \cong H^{n-1}\left(\bar{X}_{b}^{0}, Y_{b}\right)
$$

We put

$$
\begin{aligned}
& H_{\text {prim }}^{n-1}\left(\bar{X}_{b}^{0}\right):=\operatorname{Ker}\left(H^{n-1}\left(\bar{X}_{b}^{0}\right) \stackrel{r}{\rightarrow} H^{n-1}\left(Y_{b}\right)\right), \quad \text { and } \\
& H_{\text {prim }}^{n-2}\left(Y_{b}\right):=\operatorname{Coker}\left(H^{n-2}\left(\bar{X}_{b}^{0}\right) \stackrel{r}{\rightarrow} H^{n-2}\left(Y_{b}\right)\right),
\end{aligned}
$$

where $r$ is the restriction homomorphism. Then, from (5.10), we obtain an exact sequence

$$
0 \rightarrow H_{\mathrm{prim}}^{n-2}\left(Y_{b}\right) \rightarrow H_{n-1}\left(X_{b}^{0}\right) \rightarrow H_{\mathrm{prim}}^{n-1}\left(\bar{X}_{b}^{0}\right) \rightarrow 0
$$

The fundamental group $\pi_{1}(\mathcal{U}, b)$ acts on this exact sequence. The action on $H_{\mathrm{prm}}^{n-2}\left(Y_{b}\right)$ factors through the natural homomorphism

$$
\pi_{1}(U) \rightarrow \pi_{1}\left(\Gamma^{\times} \backslash \mathscr{D}_{\infty}\right) \rightarrow \pi_{1}\left(\mathbf{P}_{*}(\Gamma) \backslash D_{\infty}\right)
$$

while the action on $H_{\mathrm{prim}}^{n-1}\left(\bar{X}_{b}^{0}\right)$ factors through

$$
\pi_{1}(\mathscr{U}) \rightarrow \pi_{1}\left(\Gamma^{\times} \backslash \mathscr{D}_{0}\right) \rightarrow \pi_{1}\left(\mathbf{P}_{*}(\Gamma) \backslash D_{0}\right)
$$

Note that, by the Poincare duality, $H_{\text {prim }}^{n-1}\left(\bar{X}_{b}^{0}\right) \otimes_{\mathbf{Z}} \mathbf{Q}$ corresponds to "the module of vanishing cycles" in $H_{n-1}\left(\bar{X}_{b}^{0}\right) \otimes_{\mathbf{Z}} \mathbf{Q}$ in the sense of $[8 ; \S 3]$. Hence the classical theory of Lefschetz tells us the following:

Theorem L3. Suppose that $b \in \mathcal{U}_{N}$. Let $p$ be a value in $\mathrm{Cr}(b)$, and let $\omega$ be an element of $\mathscr{P}_{b}\left(a_{b}^{0}, p\right)$. Let $\bar{\sigma}[\omega]^{\prime} \in H_{\text {prim }}^{n-1}\left(\bar{X}_{b}^{0}\right)$ denote the image of the homology class $\bar{\sigma}[\omega] \in H_{n-1}\left(X_{b}^{0}\right)$ of a vanishing cycle $\sigma[\omega]$ for $[\omega]$ by the homomorphism in (5.11). Then $H_{\mathrm{prim}}^{n-1}\left(\bar{X}_{b}^{0}\right) \otimes_{\mathbf{Z}} \mathbf{Q}$ is generated by one element $\bar{\sigma}[\omega]^{\prime}$ as a module over the group ring $\mathbf{Q}\left[\pi_{1}\left(\mathbf{P}_{*}(\Gamma) \backslash D_{0}, \operatorname{pr}(b)\right)\right]$.

\section{\$6. Structures of $\boldsymbol{H}_{n}\left(\boldsymbol{F}_{u}\right), \boldsymbol{H}_{n}\left(\boldsymbol{F}_{u}, \partial_{0} \boldsymbol{F}_{u}\right)$ and $\boldsymbol{H}_{n}\left(\boldsymbol{F}_{u}, \partial_{\infty} \boldsymbol{F}_{u}\right)$}

In order to state the main theorem of this section, we need two definitions. First, we put 


$$
u_{\tilde{N}}:=\left\{u \in U_{N} ; \operatorname{Cr}(u) \cap \mathbb{R}_{\leq 0}=\emptyset\right\}
$$

The complement $\Gamma \backslash \mathcal{U}_{\tilde{N}}$ is a real semi-algebraic subset of real codimension $\geq 1$ in the affine space $\Gamma$. Second, we define the automorphism

$$
j: H_{n-1}\left(X_{u}^{\infty}\right) \rightarrow H_{n-1}\left(X_{u}^{\infty}\right)
$$

for $u \in U$ as follows. We set

$$
C_{u}^{0}:=\{z \in \mathbb{C} ;|z|=\varepsilon(u)\}, \text { and } C_{u}^{\infty}:=\{z \in \mathbb{C} ;|z|=1 / \varepsilon(u)\}
$$

Note that $\bar{\phi}_{u}$ has no critical values on these circles, and hence is locally trivial on them by Proposition 2.1. Then $j$ is defined as the monodromy on $H_{n-1}\left(X_{u}^{\infty}\right)$ along the loop with the base point $a_{u}^{\infty}$ which draws the circle $C_{u}^{\infty}$ in the counter-clockwise direction.

Theorem 6.1. (1) Suppose that $u \in U$. Then both of the natural homomorphisms $H_{n}\left(F_{u}\right) \rightarrow H_{n}\left(F_{u}, \partial_{0} F_{u}\right)$ and $H_{n}\left(F_{u}\right) \rightarrow H_{n}\left(F_{u}, \partial_{\infty} F_{u}\right)$ are injective. Suppose that $u \in \mathcal{U}_{N}$. Then there is a canonical isomorphism

$$
\Psi_{u}^{0}: H_{n-1}\left(X_{u}^{0}\right) \otimes \mathbb{Z}\left[q, q^{-1}\right] \stackrel{\sim}{\rightarrow} H_{n}\left(F_{u}, \partial_{0} F_{u}\right)
$$

of $\mathbb{Z}\left[q, q^{-1}\right]$-modules through which the image of $H_{n}\left(F_{u}\right) \hookrightarrow H_{n}\left(F_{u}, \partial_{0} F_{u}\right)$ is identified with $H_{n-1}\left(X_{u}^{0}\right) \otimes(1-q)$, where $(1-q) \subset \mathbb{Z}\left[q, q^{-1}\right]$ is the principal ideal generated by $1-q$. There also exists a canonical isomorphism

$$
\Psi_{u}^{\infty}: H_{n-1}\left(X_{u}^{\infty}\right) \otimes \mathbb{Z}\left[q, q^{-1}\right] \stackrel{\sim}{\rightarrow} H_{n}\left(F_{u}, \partial_{\infty} F_{u}\right)
$$

of $\mathbb{Z}\left[q, q^{-1}\right]$-modules through which the image of $H_{n}\left(F_{u}\right) \hookrightarrow H_{n}\left(F_{u}, \partial_{\infty} F_{u}\right)$ is identified with the image of the endomorphism $\mathrm{Id}-j \otimes q$ of $H_{n-1}\left(X_{u}^{\infty}\right) \otimes \mathbb{Z}\left[q, q^{-1}\right]$.

Since each of the $\mathbb{Z}\left[q, q^{-1}\right]$-modules $H_{n-1}\left(X_{u}^{0}\right) \otimes \mathbb{Z}\left[q, q^{-1}\right], H_{n-1}\left(X_{u}^{\infty}\right) \otimes \mathbb{Z}$ $\left[q, q^{-1}\right], H_{n}\left(F_{u}, \partial_{0} F_{u}\right), H_{n}\left(F_{u}, \partial_{\infty} F_{u}\right)$ and $H_{n}\left(F_{u}\right)$ forms a locally constant system of $\mathbb{Z}\left[q, q^{-1}\right]$-modules over $\mathcal{U}$, Theorem 6.1 and Proposition 5.1 imply the following : 
Corollary 6.1. For an arbitrary $u \in \mathcal{U}$, each of $H_{n}\left(F_{u}, \partial_{0} F_{u}\right), H_{n}\left(F_{u}, \partial_{\infty} F_{u}\right)$ and $H_{n}\left(F_{u}\right)$ is a free $\mathbb{Z}\left[q, q^{-1}\right]$-module of rank $N$.

Remark 6.1. The assertion that the isomorphisms $\Psi_{u}^{0}$ and $\Psi_{u}^{\infty}$ are canonical for $u \in U_{N}^{\tilde{N}}$ means that, when $u$ moves on $\mathcal{U}_{N}^{\tilde{N}}$, they form isomorphisms between the corresponding locally constant systems restricted over $\boldsymbol{U}_{N} \tilde{\text {. Even }}$ though $U_{N} \tilde{N}$ is dense in $U$, these isomorphisms of locally constant systems cannot be extended to the whole space $U$. Otherwise, the isomorphisms $\Psi_{u}^{0}$ and $\Psi_{u}^{\infty}$ would be isomorphisms of $\pi_{1}(\mathcal{U})$-modules, but this would contradict Irreducibility Theorem in Introduction, which will be proved in $\$ 10$. In particular, this argument shows that the natural homomorphism $\pi_{1}\left(U_{N}^{\tilde{N}}\right) \rightarrow \pi_{1}(\mathcal{U})$ is not surjective.

Remark 6.2. The isomorphisms $\Psi_{u}^{0}$ and $\Psi_{u}^{\infty}$ are not determined uniquely by the properties described in Theorem 6.1. For example, we can replace $\Psi_{u}^{0}$ with $q^{\nu} \cdot \Psi_{u}^{0}$ for some $\nu \in \mathbb{Z}$. In the proof, however, we will construct one specific $\Psi_{u}^{0}$ and one specific $\Psi_{u}^{\infty}$, for which Corollaries 6.2 and 6.3 below hold, and we will use $\Psi_{u}^{0}$ and $\Psi_{u}^{\infty}$ to denote these specific isomorphisms in the following.

Before starting the proof, we prepare some notation. Suppose that $u \in U$. We define

$$
\delta_{u}^{0}: I \rightarrow C_{u}^{0} \hookrightarrow \mathbb{C}^{\times} \backslash \operatorname{Cr}(u), \quad \text { and } \quad \delta_{u}^{\infty}: I \rightarrow C_{u}^{\infty} \hookrightarrow \mathbb{C}^{\times} \backslash \operatorname{Cr}(u)
$$

to be the counter-clockwise loops along the circles with the base points $a_{u}^{0}$ and $a_{u}^{\infty}$, respectively.

Remark 6.3. Then the automorphism $j: H_{n-1}\left(X_{u}^{\infty}\right) \rightarrow H_{n-1}\left(X_{u}^{\infty}\right)$ is nothing but the monodromy operator $\left[\delta_{u}^{\infty}\right]_{*}$. On the other hand, since $\bar{\phi}_{u}: \mathbf{A}^{n} \rightarrow \mathbf{C}$ is locally trivial on the close disk $\Delta_{\varepsilon(u)}(0)=B_{u}^{0} \cup\{0\}$, the monodromy action $\left[\delta_{u}^{0}\right]_{*}$ : $H_{n-1}\left(X_{u}^{0}\right) \rightarrow H_{n-1}\left(X_{u}^{0}\right)$ is the identity by Proposition 2.1. (See (5.5).)

We put

$$
R_{u}^{0}:=e^{-1}\left(C_{u}^{0}\right)=\log \varepsilon(u)+\sqrt{-1} \mathbf{R}, \quad R_{u}^{\infty}:=e^{-1}\left(C_{u}^{\infty}\right)=\log \varepsilon(u)^{-1}+\sqrt{-1} \mathbf{R},
$$

and

$$
Z_{u}^{0}:=e^{-1}\left(a_{u}^{0}\right)=\log \varepsilon(u)+\sqrt{-1} \mathbb{Z}, \quad Z_{u}^{\infty}:=e^{-1}\left(a_{u}^{\infty}\right)=\log \varepsilon(u)^{-1}+\sqrt{-1} \mathbf{Z},
$$


where $e: \mathbb{C} \rightarrow \mathbb{C}^{\times}$is the exponential map. For each $\nu \in \mathbb{Z}$, we put

$$
a_{u}^{0}\langle\nu\rangle:=\log \varepsilon(u)+\sqrt{-1} \nu \in Z_{u}^{0} \text {, and } a_{u}^{\infty}\langle\nu\rangle:=\log \varepsilon(u)^{-1}+\sqrt{-1} \nu \in Z_{u}^{\infty} \text {. }
$$

We also put

$$
X_{u}^{0}\langle\nu\rangle:=\phi_{u}^{-1}\left(a_{u}^{0}\langle\nu\rangle\right), \text { and } X_{u}^{\infty}\langle\nu\rangle:=\phi_{u}^{-1}\left(a_{u}^{\infty}\langle\nu\rangle\right)
$$

Then we have the natural isomorphisms

$$
X_{u}^{0}\langle\nu\rangle \cong X_{u}^{0}, \text { and } \quad X_{u}^{\infty}\langle\nu\rangle \cong X_{u}^{\infty}
$$

induced from the covering map $\tilde{e}: F_{u} \rightarrow E_{u}$.

Now suppose that $u \in \mathcal{U}_{N}$. For each $\nu \in \mathbb{Z}$, there exists a unique connected component $\left(\mathbb{C} \backslash \mathbb{R}_{\leq 0}\right)\langle\nu\rangle$ of $e^{-1}\left(\mathbb{C} \backslash \mathbb{R}_{\leq 0}\right)$ which contains $a_{u}^{0}\langle\nu\rangle$ and $a_{u}^{\infty}\langle\nu\rangle$. Let $\left\{p_{1}, \ldots, p_{N}\right\}$ be the set $\operatorname{Cr}(u)$, which is contained in $\mathbb{C} \backslash \mathbb{R} \leq 0$. For each $\nu \in \mathbb{Z}$, let $p_{\imath}\langle\nu\rangle$ denote the unique point on $\left(\mathbb{C} \backslash \mathbb{R} \leq_{0}\right)\langle\nu\rangle$ which is mapped to $p_{\imath}$ by $e$. Therefore, we have

$$
\mathscr{C} r(u)=\coprod_{\nu \in \mathbb{Z}} \operatorname{Cr}(u)\langle\nu\rangle
$$

where

$$
\operatorname{Cr}(\imath)\langle\nu\rangle:=\left\{p_{\imath}\langle\nu\rangle ; i=1, \ldots, N\right\}
$$

Note that $-\pi<\arg p_{\imath}<\pi$ for $i=1, \ldots, N$. We put

$$
\eta(u):=\frac{1}{2} \min \left\{\pi-\arg p_{\imath}, \pi+\arg p_{\imath} ; i=1, \ldots, N\right\}
$$

Then $\eta: \mathscr{U}_{N} \rightarrow \mathbb{R}_{>0}$ is a continuous function on $\mathcal{U}_{N}^{\tilde{N}}$. We put

$$
K_{u}:=\left\{z \in \mathbb{C}^{\times} ; \varepsilon(u) \leq|z| \leq \varepsilon(u)^{-1} \text {, and }-\pi+\eta(u) \leq \arg z \leq \pi-\eta(u)\right\} \text {, }
$$

and

$K_{u}\langle\nu\rangle:=$ the unique connected component of $e^{-1}\left(K_{u}\right)$ containing $a_{u}^{0}\langle\nu\rangle$ and $a_{u}^{\infty}\langle\nu\rangle$.

Then, for each $\nu$, the exponential map $e: \mathbb{C} \rightarrow \mathbb{C}^{\times}$induces an isomorphism between $K_{u}\langle\nu\rangle$ and $K_{u}$, and $e^{-1}\left(K_{u}\right)$ is the disjoint union of all $K_{u}\langle\nu\rangle$. Moreover, 
each $\operatorname{Cr}(u)\langle\nu\rangle$ is contained in the interior of $K_{u}\langle\nu\rangle$. We put

$$
\begin{aligned}
& M_{u}^{0}:=e^{-1}\left(K_{u} \cup C_{u}^{0}\right)=\left(\coprod_{\nu \in \mathbf{Z}} K_{u}\langle\nu\rangle\right) \cup R_{u}^{0} \subset \mathbf{C}, \text { and } \\
& M_{u}^{\infty}:=e^{-1}\left(K_{u} \cup C_{u}^{\infty}\right)=\left(\coprod_{\nu \in Z} K_{u}\langle\nu\rangle\right) \cup R_{u}^{\infty} \subset \mathbf{C} .
\end{aligned}
$$

We also put

$$
N_{u}^{0}:=K_{u} \cap C_{u}^{0}, \quad \text { and } \quad N_{u}^{\infty}:=K_{u} \cap C_{u}^{\infty} \text {, }
$$

both of which are $\operatorname{arcs}$ in $\mathbb{C}^{\times}$. Each $K_{u}\langle\nu\rangle$ is a rectangle in $\mathbb{C}$, whose vertical sides are given by

$$
N_{u}^{0}\langle\nu\rangle:=K_{u}\langle\nu\rangle \cap R_{u}^{0}, \quad \text { and } \quad N_{u}^{\infty}\langle\nu\rangle:=K_{u}\langle\nu\rangle \cap R_{u}^{\infty}
$$

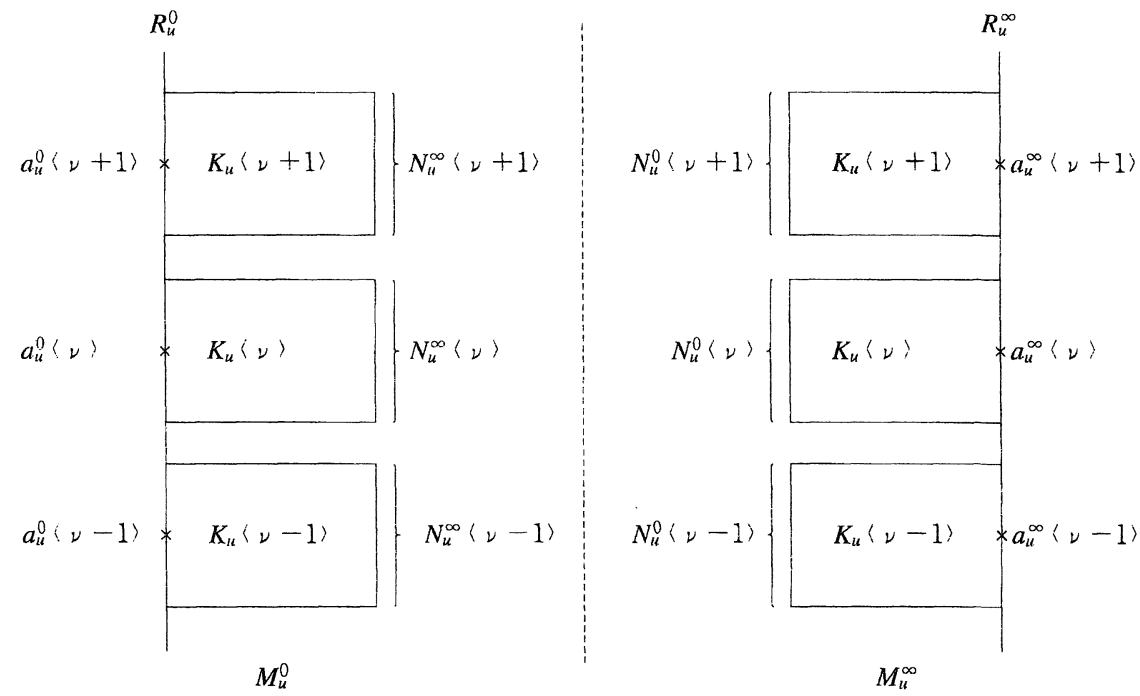

Figure 5

Then we have

$$
e^{-1}\left(N_{u}^{0}\right)=\coprod_{\nu \in \mathbb{Z}} N_{u}^{0}\langle\nu\rangle \text {, and } e^{-1}\left(N_{u}^{\infty}\right)=\coprod_{\nu \in \mathbb{Z}} N_{u}^{\infty}\langle\nu\rangle \text {. }
$$

Proof of Theorem 6.1. We will give a proof only to the assertions con- 
cerned with $H_{n}\left(F_{u}, \partial_{\infty} F_{u}\right)$ and $\Psi_{u}^{\infty}$. The assertions concerned with $H_{n}\left(F_{u}\right.$, $\left.\partial_{0} F_{u}\right)$ and $\Psi_{u}^{0}$ can be proved completely in the same way. All we have to do is just to replace every $\infty$ appearing in the argument with 0 , and to notice that the monodromy action on $H_{n-1}\left(X_{u}^{0}\right)$ associated to the loop $\delta_{u}^{0}$ is the identity. (See Remark 6.3.)

Since $\phi_{u}$ has no critical values in $e^{-1}\left(B_{u}^{\infty}\right)$, and $e^{-1}\left(B_{u}^{\infty}\right)$ is contractible, Proposition 3.2 implies that the inclusion $X_{u}^{\infty}\langle\nu\rangle \hookrightarrow \psi_{u}^{-1}\left(e^{-1}\left(B_{u}^{\infty}\right)\right)=\partial_{\infty} F_{u}$ in duces homotopy equivalence. Combining this with (6.1) and Theorem L2, we have $H_{n}\left(\partial_{\infty} F_{u}\right) \cong H_{n}\left(X_{u}^{\infty}\right)=0$. Thus the natural homomorphism $H_{n}\left(F_{u}\right) \rightarrow H_{n}\left(F_{u}\right.$, $\left.\partial_{\infty} F_{u}\right)$ is injective.

Now suppose that $u \in \mathcal{U}_{N}^{\tilde{N}}$. The pair $\left(M_{u}^{\infty}, R_{u}^{\infty}\right)$ is a strong deformation retract of the pair $\left(\mathbb{C}, e^{-1}\left(B_{u}^{\infty}\right)\right)$. Since $\mathscr{C} r(u)$ is contained in the interior of $M_{u}^{\infty}$, Proposition 3.2 implies that the inclusion

$$
\left(\phi_{u}^{-1}\left(M_{u}^{\infty}\right), \phi_{u}^{-1}\left(R_{u}^{\infty}\right)\right) \hookrightarrow\left(F_{u}, \partial_{\infty} F_{u}\right)
$$

induces homotopy equivalence, and there exists a strong deformation retraction

$$
\left(F_{u}, \partial_{\infty} F_{u}\right) \rightarrow\left(\phi_{u}^{-1}\left(M_{u}^{\infty}\right), \phi_{u}^{-1}\left(R_{u}^{\infty}\right)\right)
$$

which is the homotopy inverse of the inclusion. Note that the deck transformation $T_{u}$ on $\left(F_{u}, \partial_{\infty} F_{u}\right)$ induces an automorphism of the pair of subspaces $\left(\phi_{u}^{-1}\right.$ $\left.\left(M_{u}^{\infty}\right), \phi_{u}^{-1}\left(R_{u}^{\infty}\right)\right)$. Thus both of $H_{n}\left(\phi_{u}^{-1}\left(M_{u}^{\infty}\right)\right)$ and $H_{n}\left(\phi_{u}^{-1}\left(M_{u}^{\infty}\right), \phi_{u}^{-1}\left(R_{u}^{\infty}\right)\right)$ can be considered as $\mathbb{Z}\left[q, q^{-1}\right]$-modules, and we obtain a commutative diagram of $\mathbb{Z}\left[q, q^{-1}\right]$-modules ;

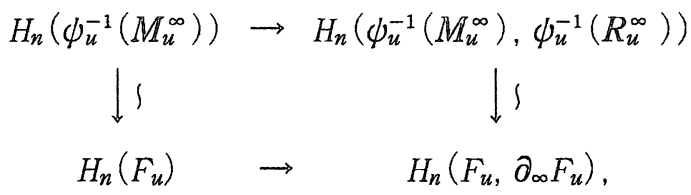

where the horizontal arrows are the natural homomorphisms and the vertical arrows are the isomorphisms induced by the inclusions. By the excision property of homology groups, we have

$$
\begin{aligned}
H_{n}\left(\phi_{u}^{-1}\left(M_{u}^{\infty}\right), \phi_{u}^{-1}\left(R_{u}^{\infty}\right)\right) & \cong H_{n}\left(\phi_{u}^{-1}\left(\coprod_{\nu \in \mathbb{Z}} K_{u}\langle\nu\rangle\right), \phi_{u}^{-1}\left(\coprod_{\nu \in \mathbb{Z}} N_{u}^{\infty}\langle\nu\rangle\right)\right) \\
& \cong \bigoplus_{\nu \in \mathbb{Z}} H_{n}\left(\phi_{u}^{-1}\left(K_{u}\langle\nu\rangle\right), \phi_{u}^{-1}\left(N_{u}^{\infty}\langle\nu\rangle\right)\right) .
\end{aligned}
$$


On the other hand, the deck transformation $T_{u}$ on $\left(F_{u}, \partial_{\infty} F_{u}\right)$ induces isomorphisms

$$
\left(\phi_{u}^{-1}\left(K_{u}\langle\nu\rangle\right), \phi_{u}^{-1}\left(N_{u}^{\infty}\langle\nu\rangle\right)\right) \stackrel{\sim}{\rightarrow}\left(\phi_{u}^{-1}\left(K_{u}\langle\nu+1\rangle\right), \phi_{u}^{-1}\left(N_{u}^{\infty}\langle\nu+1\rangle\right)\right)
$$

for all $\nu \in \mathbb{Z}$, and these isomorphisms are compatible with the isomorphisms

$$
\left(\phi_{u}^{-1}\left(K_{u}\langle\nu\rangle\right), \phi_{u}^{-1}\left(N_{u}^{\infty}\langle\nu\rangle\right)\right) \cong\left(\phi_{u}^{-1}\left(K_{u}\right), \phi_{u}^{-1}\left(N_{u}^{\infty}\right)\right)
$$

given by the restriction of the covering map $\tilde{e}: F_{u} \rightarrow E_{u}$. Hence the multiplication by $q$ in the decomposition (6.5) into the direct sum is given by the shift of the numbering $\langle\nu\rangle$;

$$
H_{n}\left(\phi_{u}^{-1}\left(K_{u}\langle\nu\rangle\right), \phi_{u}^{-1}\left(N_{u}^{\infty}\langle\nu\rangle\right)\right) \stackrel{\sim}{\rightarrow} H_{n}\left(\phi_{u}^{-1}\left(K_{u}\langle\nu+1\rangle\right), \phi_{u}^{-1}\left(N_{u}^{\infty}\langle\nu+1\rangle\right)\right),
$$

which commutes with the isomorphisms

$$
H_{n}\left(\phi_{u}^{-1}\left(K_{u}\langle\mu\rangle\right), \phi_{u}^{-1}\left(N_{u}^{\infty}\langle\mu\rangle\right)\right) \cong H_{n}\left(\phi_{u}^{-1}\left(K_{u}\right), \phi_{u}^{-1}\left(N_{u}^{\infty}\right)\right)
$$

for $\mu=\nu$ and $\mu=\nu+1$ induced by (6.6).

Therefore, we get a unique isomorphism of $\mathbb{Z}\left[q, q^{-1}\right]$-modules

$$
H_{n}\left(\phi_{u}^{-1}\left(M_{u}^{\infty}\right), \phi_{u}^{-1}\left(R_{u}^{\infty}\right)\right) \cong H_{n}\left(\phi_{u}^{-1}\left(K_{u}\right), \phi_{u}^{-1}\left(N_{u}^{\infty}\right)\right) \otimes \mathbb{Z}\left[q, q^{-1}\right]
$$

characterized by the commutativity of the following diagram for all $\nu \in \mathbb{Z}$ :

$$
\begin{array}{ccc}
H_{n}\left(\phi_{u}^{-1}\left(K_{u}\langle\nu\rangle\right), \phi_{u}^{-1}\left(N_{u}^{\infty}\langle\nu\rangle\right)\right) & \underset{\text { by }(6.5)}{\hookrightarrow} & H_{n}\left(\phi_{u}^{-1}\left(M_{u}^{\infty}\right), \phi_{u}^{-1}\left(R_{u}^{\infty}\right)\right) \\
\lfloor\text {; by (66) } & & \\
H_{n}\left(\phi_{u}^{-1}\left(K_{u}\right), \phi_{u}^{-1}\left(N_{u}^{\infty}\right)\right) & & \\
\cong H_{n}\left(\phi_{u}^{-1}\left(K_{u}\right), \phi_{u}^{-1}\left(N_{u}^{\infty}\right)\right) \cdot q^{\nu} & \hookrightarrow & H_{n}\left(\phi_{u}^{-1}\left(K_{u}\right), \phi_{u}^{-1}\left(N_{u}^{\infty}\right)\right) \otimes \mathbb{Z}\left[q, q^{-1}\right] .
\end{array}
$$

This characterization of (6.7) will determine the specific isomorphism $\Psi_{u}^{\infty}$ mentioned in Remark 6.2.

On the other hand, since $K_{u} \subset \mathbf{C}$ is a strong deformation retract of $\mathbb{C}$, which contains all of the critical values $\operatorname{Cr}(u)$ of $\widehat{\phi}_{u}: \mathbf{A}^{n} \rightarrow \mathbf{C}$ in its interior, the pull-back $\phi_{u}^{-1}\left(K_{u}\right)=\widehat{\phi}_{u}^{-1}\left(K_{u}\right)$ is also a strong deformation retract of $\mathbf{A}^{n}$ by Proposition 2.1. Combining this with the isomorphisms (6.6), we see that 


$$
\phi_{u}^{-1}\left(K_{u}\right) \text { and } \phi_{u}^{-1}\left(K_{u}\langle\nu\rangle\right) \text { are all contractible spaces. }
$$

This implies that we get isomorphisms

$$
\begin{aligned}
& H_{n}\left(\phi_{u}^{-1}\left(K_{u}\right), \phi_{u}^{-1}\left(N_{u}^{\infty}\right)\right) \stackrel{\sim}{\rightarrow} H_{n-1}\left(\phi_{u}^{-1}\left(N_{u}^{\infty}\right)\right), \text { and } \\
& H_{n}\left(\phi_{u}^{-1}\left(K_{u}\langle\nu\rangle\right), \phi_{u}^{-1}\left(N_{u}^{\infty}\langle\nu\rangle\right)\right) \stackrel{\sim}{\rightarrow} H_{n-1}\left(\phi_{u}^{-1}\left(N_{u}^{\infty}\langle\nu\rangle\right)\right)
\end{aligned}
$$

induced by the boundary homomorphisms. Combining these with (6.5) and (6.7), we obtain the isomorphisms

$$
\begin{aligned}
H_{n}\left(\phi_{u}^{-1}\left(M_{u}^{\infty}\right), \phi_{u}^{-1}\left(R_{u}^{\infty}\right)\right) & \cong \bigoplus_{\nu \in \mathbb{Z}} H_{n-1}\left(\phi_{u}^{-1}\left(N_{u}^{\infty}\langle\nu\rangle\right)\right) \\
& \cong H_{n-1}\left(\phi_{u}^{-1}\left(N_{u}^{\infty}\right)\right) \otimes \mathbb{Z}\left[q, q^{-1}\right]
\end{aligned}
$$

of $\mathbb{Z}\left[q, q^{-1}\right]$-modules. Now, since $\phi_{u}$ and $\phi_{u}$ are locally trivial over the arc $N_{u}^{\infty}$ $\subset \mathbb{C}^{\times}$and the line segment $N_{u}^{\infty}\langle\nu\rangle \subset \mathbb{C}$, respectively, the inclusions

$$
X_{u}^{\infty} \hookrightarrow \phi_{u}^{-1}\left(N_{u}^{\infty}\right) \quad \text { and } \quad X_{u}^{\infty}\langle\nu\rangle \hookrightarrow \phi_{u}^{-1}\left(N_{u}^{\infty}\langle\nu\rangle\right)
$$

induce homotopy equivalences. Therefore (6.10) can be written as

$$
\begin{aligned}
H_{n}\left(\phi_{u}^{-1}\left(M_{u}^{\infty}\right), \phi_{u}^{-1}\left(R_{u}^{\infty}\right)\right) & \cong \bigoplus_{\nu \in \mathbb{Z}} H_{n-1}\left(X_{u}^{\infty}\langle\nu\rangle\right) \\
& \cong H_{n-1}\left(X_{u}^{\infty}\right) \otimes \mathbb{Z}\left[q, q^{-1}\right] .
\end{aligned}
$$

Note that, because of the characterization of (6.7), an element $\tilde{x}_{2}$ of the direct summand $H_{n-1}\left(X_{n}^{\infty}\langle\nu\rangle\right)$ corresponds via (6.12) to $x_{\nu} \otimes q^{\nu} \in H_{n-1}\left(X_{u}^{\infty}\right) \otimes \mathbb{Z}[q$, $\left.q^{-1}\right]$, where $x_{\nu} \in H_{n-1}\left(X_{u}^{\infty}\right)$ is the image of $\tilde{x}_{\nu}$ by the isomorphism $H_{n-1}\left(X_{u}^{\infty}\langle\nu\rangle\right)$ $\cong H_{n-1}\left(X_{u}^{\infty}\right)$ induced from (6.1). Combining this with (6.4), we get the desired isomorphism $\Psi_{u}^{\infty}$ of $\mathbb{Z}\left[q, q^{-1}\right]$-modules. Note that the homeomorphism types of all spaces and continuous maps which have appeared in the course of the construction of $\Psi_{u}^{\infty}$ do not change when $u$ varies continuously in $\mathscr{U}_{N}^{\tilde{N}}$. Hence the isomorphisms $\Psi_{u}^{\infty}$ with $u \in \mathcal{U}_{N}^{\tilde{N}}$ yield an isomorphism between the corresponding locally constant systems over $\tilde{U}_{N}^{\tilde{N}}$.

Now we shall calculate $H_{n}\left(F_{u}\right) \cong H_{n}\left(\phi_{u}^{-1}\left(M_{u}^{\infty}\right)\right)$ by applying the MayerVietoris sequence to the decomposition.

$$
\phi_{u}^{-1}\left(M_{u}^{\infty}\right)=\phi_{u}^{-1}\left(\coprod_{\nu \in \mathbb{Z}} K_{u}\langle\nu\rangle\right) \cup \phi_{u}^{-1}\left(R_{u}^{\infty}\right)
$$


Note that

$$
\phi_{u}^{-1}\left(\coprod_{\nu \in \mathbf{Z}} K_{u}\langle\nu\rangle\right) \cap \phi_{u}^{-1}\left(R_{u}^{\infty}\right)=\coprod_{\nu \in \mathbf{Z}} \phi_{u}^{-1}\left(N_{u}^{\infty}\langle\nu\rangle\right)
$$

Since $\phi_{u}^{-1}\left(K_{u}\langle\nu\rangle\right)$ is contractible for each $\nu \in \mathbb{Z}$ by (6.8), the Mayer-Vietoris sequence is of the form

$$
\begin{aligned}
& \rightarrow \bigoplus_{\nu \in \mathbf{Z}} H_{n}\left(\phi_{u}^{-1}\left(N_{u}^{\infty}\langle\nu\rangle\right)\right) \rightarrow H_{n}\left(\phi_{u}^{-1}\left(R_{u}^{\infty}\right)\right) \rightarrow H_{n}\left(\phi_{u}^{-1}\left(M_{u}^{\infty}\right)\right) \\
& \vec{a} \bigoplus_{\nu \in \mathbf{Z}} H_{n-1}\left(\phi_{u}^{-1}\left(N_{u}^{\infty}\langle\nu\rangle\right)\right) \underset{c}{\rightarrow} H_{n-1}\left(\phi_{u}^{-1}\left(R_{u}^{\infty}\right)\right) \rightarrow \\
& \cdots
\end{aligned}
$$

Recall the construction of the isomorphism (6.10). It has been derived from (6.5) through the boundary map (6.9). Then it can be easily checked that the following diagram is commutative ;

$$
\begin{array}{ccc}
H_{n}\left(\phi_{u}^{-1}\left(M_{u}^{\infty}\right)\right) & \stackrel{\partial \text { in }(6.13)}{\rightarrow} & \bigoplus_{\nu \in \mathbf{Z}} H_{n-1}\left(\phi_{u}^{-1}\left(N_{u}^{\infty}\langle\nu\rangle\right)\right) \\
(6.4) \downarrow \rho & & (6.4) \text { and }(6.10) \uparrow / \\
H_{n}\left(F_{u}\right) & \stackrel{\text { the natural map }}{\rightarrow} & H_{n}\left(F_{u}, \partial_{\infty} F_{u}\right) .
\end{array}
$$

Hence the image of the injection $H_{n}\left(F_{u}\right) \rightarrow H_{n}\left(F_{u}, \partial_{\infty} F_{u}\right)$ is identified, via (6.4) and (6.10), with the kernel of the homomorphism

$$
\iota: \bigoplus_{\nu \in \mathbf{Z}} H_{n-1}\left(\phi_{u}^{-1}\left(N_{u}^{\infty}\langle\nu\rangle\right)\right) \rightarrow H_{n-1}\left(\phi_{u}^{-1}\left(R_{u}^{\infty}\right)\right)
$$

in (6.13) induced by the inclusions. Since $\phi_{u}$ has no critical values on $R_{u}^{\infty}$, the inclusion $X_{u}^{\infty}\langle\nu\rangle \hookrightarrow \phi_{u}^{-1}\left(R_{u}^{\infty}\right)$ induces homotopy equivalence for each $\nu \in \mathbb{Z}$. Let

$$
\phi_{u}^{-1}\left(R_{u}^{\infty}\right) \rightarrow X_{u}^{\infty}\langle 0\rangle
$$

be a continuous map which represents the homotopy inverse of the inclusion $X_{u}^{\infty}\langle 0\rangle \hookrightarrow \phi_{u}^{-1}\left(R_{u}^{\infty}\right)$. Consider the composite

$$
X_{u}^{\infty} \underset{(6.1)}{\stackrel{\sim}{\rightarrow}} X_{u}^{\infty}\langle\nu\rangle \hookrightarrow \psi_{u}^{-1}\left(R_{u}^{\infty}\right) \underset{(6.14)}{\rightarrow} X_{u}^{\infty}\langle 0\rangle \underset{(6.1)}{\stackrel{\sim}{\rightarrow}} X_{u}^{\infty},
$$


of continuous maps, each of which induces homotopy equivalence. The induced automorphism $H_{n-1}\left(X_{u}^{\infty}\right) \rightarrow H_{n-1}\left(X_{u}^{\infty}\right)$ is nothing but the monodromy operator $j^{-\nu}$, because the path on $\mathbb{C}$ from $a_{u}^{\infty}\langle\nu\rangle$ to $a_{u}^{\infty}\langle 0\rangle$ along $R_{u}^{\infty}$ is mapped to the loop $\left(\delta_{u}^{\infty}\right)^{-\nu}$ on $\mathbb{C}^{\times}$by $e$. Therefore, through the isomorphisms

$$
\underset{\nu \in \mathbb{Z}}{\bigoplus} H_{n-1}\left(\phi_{u}^{-1}\left(N_{u}^{\infty}\langle\nu\rangle\right)\right) \underset{(6.11)}{\cong} \underset{\nu \in \mathbb{Z}}{\bigoplus} H_{n-1}\left(X_{u}^{\infty}\langle\nu\rangle\right) \underset{(6.12)}{\cong} H_{n-1}\left(X_{u}^{\infty}\right) \otimes \mathbb{Z}\left[q, q^{-1}\right]
$$

and

$$
H_{n-1}\left(\phi_{u}^{-1}\left(R_{u}^{\infty}\right)\right) \underset{(\epsilon .14)}{\cong} H_{n-1}\left(X_{u}^{\infty}\langle 0\rangle\right) \underset{(6.1)}{\cong} H_{n-1}\left(X_{u}^{\infty}\right)
$$

we can indentify $\iota$ in $(6.13)$ with $\tilde{\iota}: H_{n-1}\left(X_{u}^{\infty}\right) \otimes \mathbb{Z}\left[q, q^{-1}\right] \rightarrow H_{n-1}\left(X_{u}^{\infty}\right)$ given by

$$
\tilde{\iota}\left(\sum_{\nu \in \mathbb{Z}}\left(x_{\nu} \otimes q^{\nu}\right)\right)=\sum_{\nu \in \mathbb{Z}} j^{-\nu}\left(x_{\nu}\right), \text { where } x_{\nu} \in H_{n-1}\left(X_{u}^{\infty}\right)
$$

because an element $x_{\nu} \otimes q^{\nu}$ of $H_{n-1}\left(X_{u}^{\infty}\right) \otimes \mathbb{Z}\left[q, q^{-1}\right]$ corresponds to an element of the direct summand $H_{n-1}\left(\phi_{u}^{-1}\left(N_{u}^{\infty}\langle\nu\rangle\right)\right) \cong H_{n-1}\left(X_{u}^{\infty}\langle\nu\rangle\right)$ via $(6.15)$ by the characterization of (6.7) above. Then it can be easily checked that the kernel of this $\tilde{i}$ coincides with the image of the endomorphism Id $-j \otimes q$. Since $\Psi_{u}^{\infty}$ is given by (6.15) combined with $(6.10),(6.12)$ and (6.4), we complete the proof.

By looking back at the constructions and taking the characterization of (6.7) into account, we can descride the isomorphisms $\Psi_{u}^{0}$ and $\Psi_{u}^{\infty}$ in a geometric way.

Corollary 6.2. Let $\Lambda$ be an $(n-1)$-cycle in $X_{u}^{0}\left(\right.$ resp. $\left.X_{u}^{\infty}\right)$. Let $\Gamma_{\nu}$ be an $n$-chain in $\phi_{u}^{-1}\left(K_{u}\langle\nu\rangle\right)$ such that $\partial \Gamma_{\nu} \subset X_{u}^{0}\langle\nu\rangle\left(\right.$ resp. $\left.\partial \Gamma_{\nu} \subset X_{u}^{\infty}\langle\nu\rangle\right)$ and that $\left[\partial \Gamma_{\nu}\right]$ is mapped to $[\Lambda]$ via the isomorphism $H_{n-1}\left(X_{u}^{0}\langle\nu\rangle\right) \cong H_{n-1}\left(X_{u}^{0}\right)$ (resp. $H_{n-1}$ $\left.\left(X_{u}^{\infty}\langle\nu\rangle\right) \cong H_{n-1}\left(X_{u}^{\infty}\right)\right)$ induced by (6.1). Then

$$
\left[\Gamma_{\nu}\right]=\Psi_{u}^{0}\left([\Lambda] \otimes q^{\nu}\right) \quad\left(\operatorname{resp.}\left[\Gamma_{\nu}\right]=\Psi_{u}^{\infty}\left([\Lambda] \otimes q^{\nu}\right)\right)
$$

holds in $H_{n}\left(F_{u}, \partial_{0} F_{u}\right)$ (resp. in $\left.H_{n}\left(F_{u}, \partial_{\infty} F_{u}\right)\right)$. In particular, let $\Gamma$ be an $n$-chain

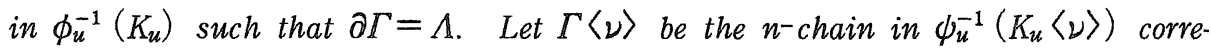


sponding to $\Gamma$ via the isomorphism (6.6). Then $[\Gamma\langle\nu\rangle]=\Psi_{u}^{0}\left([\Lambda] \otimes q^{\nu}\right)($ resp. $\left.[\Gamma\langle\nu\rangle]=\Psi_{u}^{\infty}\left([\Lambda] \otimes q^{\nu}\right)\right)$.

Remark 6.4. Since $\phi_{u}^{-1}\left(K_{u}\right)$ is contractible, there always exists an $n$-chain $\Gamma \subset \phi_{u}^{-1}\left(K_{u}\right)$ such that $\partial \Gamma=\Lambda$ for any $(n-1)$-cycle $\Lambda \subset X_{u}^{0}\left(\right.$ resp. $\left.\Lambda \subset X_{u}^{\infty}\right)$.

Corollary 6.3. Suppose, the other way around, that we are given an $n$-cycle $\Gamma$ in $\left(F_{u}, \partial_{0} F_{u}\right)\left(\right.$ resp. in $\left.\left(F_{u}, \partial_{\infty} F_{u}\right)\right)$. Let $\Gamma^{\prime}$ be the image of $\Gamma$ by the retraction

$$
\left(F_{u}, \partial_{0} F_{u}\right) \rightarrow\left(\phi_{u}^{-1}\left(M_{u}^{0}\right), \phi_{u}^{-1}\left(R_{u}^{0}\right)\right)\left(\operatorname{resp} .\left(F_{u}, \partial_{\infty} F_{u}\right) \rightarrow\left(\phi_{u}^{-1}\left(M_{u}^{\infty}\right), \phi_{u}^{-1}\left(R_{u}^{\infty}\right)\right)\right)
$$

which is the homotopy inverse of the inclusion. We put $\Gamma_{\nu}^{\prime}:=\Gamma^{\prime} \cap \phi_{u}^{-1}\left(K_{u}\langle\nu\rangle\right)$. Then, since $\partial \Gamma^{\prime} \subset \phi_{u}^{-1}\left(R_{u}^{0}\right) \quad\left(\right.$ resp. $\left.\partial \Gamma^{\prime} \subset \phi_{u}^{-1}\left(R_{u}^{\infty}\right)\right)$, we have $\partial \Gamma_{\nu}^{\prime} \subset \phi_{u}^{-1}\left(N_{u}^{0}\langle\nu\rangle\right)$ (resp. $\left.\partial \Gamma_{\nu}^{\prime} \subset \psi_{u}^{-1}\left(N_{u}^{\infty}\langle\nu\rangle\right)\right) . \quad$ Let $\Lambda_{\nu} \subset X_{u}^{0}\left(\right.$ resp. $\left.\Lambda_{\nu} \subset X_{u}^{\infty}\right)$ be the image of $\partial \Gamma_{\nu}^{\prime}$ by the continuos map

$$
\phi_{u}^{-1}\left(N_{u}^{0}\langle\nu\rangle\right) \cong \phi_{u}^{-1}\left(N_{u}^{0}\right) \underset{\mathrm{rt}}{\rightarrow} X_{u}^{0}, \quad\left(\operatorname{resp} \cdot \phi_{u}^{-1}\left(N_{u}^{\infty}\langle\nu\rangle\right) \cong \phi_{u}^{-1}\left(N_{u}^{\infty}\right) \underset{\mathrm{rt}}{\rightarrow} X_{u}^{\infty},\right)
$$

where $\mathrm{rt}$ is the homotopy inverse of the inclusion. Then

$$
[\Gamma]=\Psi_{u}^{0}\left(\sum_{\nu \in \mathbb{Z}}\left(\left[\Lambda_{\nu}\right] \otimes q^{\nu}\right)\right) \quad\left(\text { resp. }[\Gamma]=\Psi_{u}^{\infty}\left(\sum_{\nu \in \mathbb{Z}}\left(\left[\Lambda_{\nu}\right] \otimes q^{\nu}\right)\right)\right)
$$

holds in $H_{n}\left(F_{u}, \partial_{0} F_{u}\right) \quad$ (resp. in $\left.H_{n}\left(F_{u}, \partial_{\infty} F_{u}\right)\right)$.

From now on, we consider $H_{n}\left(F_{u}\right)$ as $\mathbb{Z}\left[q, q^{-1}\right]$-submodules of $H_{n}\left(F_{u}, \partial_{0} F_{u}\right)$ and of $H_{n}\left(F_{u}, \partial_{\infty} F_{u}\right)$. For $u \in \mathcal{U}_{N}^{\sim}$, we put

$$
\tilde{q}:=\Psi_{u}^{\infty} \circ(j \otimes q) \circ\left(\Psi_{u}^{\infty}\right)^{-1}: H_{n}\left(F_{u}, \partial_{\infty} F_{u}\right) \rightarrow H_{n}\left(F_{u}, \partial_{\infty} F_{u}\right)
$$

Then we have

$$
H_{n}\left(F_{u}\right)=(1-q) H_{n}\left(F_{u}, \partial_{0} F_{u}\right), \quad \text { and } \quad H_{n}\left(F_{u}\right)=(1-\tilde{q}) H_{n}\left(F_{u}, \partial_{\infty} F_{u}\right)
$$

Corollary 6.4. The natural maps induce isomorphisms of $\mathbf{Q}(q)\left[\pi_{1}(\mathcal{U}, b)\right]$ modules

$$
H_{n}\left(F_{b}\right) \otimes_{\mathbf{Z}\left[q, q^{-1}\right]} \mathbf{Q}(q) \cong H_{n}\left(F_{b}, \partial_{0} F_{b}\right) \otimes_{\mathbf{Z}\left[q, q^{-1}\right]} \mathbf{Q}(q) \cong H_{n}\left(F_{b}, \partial_{\infty} F_{b}\right) \otimes_{\mathbf{Z}\left[q, q^{-1}\right]} \mathbf{Q}(q)
$$


where $\mathbb{Q}(q)$ is the quotient field of $\mathbb{Z}\left[q, q^{-1}\right]$, and $\mathbb{Q}(q)\left[\pi_{1}(U, b)\right]$ is the group ring of $\pi_{1}(U, b)$ with coefficients in $\mathbb{Q}(q)$.

Proof. The first isomorphism is obvious from (6.16). Note that the endomorphism Id $-j \otimes q$ on the vector space $H_{n-1}\left(X_{b}^{\infty}\right) \otimes_{\mathbb{Z}} \mathbb{Q}(q)$ over $\mathbb{Q}(q)$ is invertible. Hence the second isomorphism also holds.

The following Lemma 6.1 will be used in $\$ 10$.

Lemma 6.1. Suppose that $u \in \mathcal{U}_{N}^{\tilde{N}}$. Suppose that an element $\lambda \in H_{n-1}\left(X_{u}^{0}\right)$ is given. Then there exist elements $\lambda_{0}, \lambda_{1} \in H_{n-1}\left(X_{u}^{\infty}\right)$ such that

$$
(1-q) \Psi_{u}^{0}(\lambda \otimes 1)=\Psi_{u}^{\infty}\left(\lambda_{0} \otimes 1\right)+\Psi_{u}^{\infty}\left(\lambda_{1} \otimes q\right)
$$

holds in $H_{n}\left(F_{u}\right)$.

Proof. First we shall describe an $n$-cycle in $F_{u}$ which represents the homology class

$$
(1-q) \Psi_{u}^{0}\left(\lambda \otimes q^{\nu}\right) \in H_{n}\left(F_{u}\right)
$$

Let $\Lambda \subset X_{u}^{0}$ be an $(n-1)$-cycle which represents $\lambda$, and let $\Lambda\langle\nu\rangle \subset X_{u}^{0}\langle\nu\rangle$ be the lifting of $\Lambda$ by (6.1). By Remark 6.4, we have an $n$-chain $\Gamma$ in $\phi_{u}^{-1}\left(K_{u}\right)$ such that its lifting $\Gamma\langle\nu\rangle \subset \phi_{u}^{-1}\left(K_{u}\langle\nu\rangle\right)$ satisfies $\partial \Gamma\langle\nu\rangle=\Lambda\langle\nu\rangle$ for all $\nu \in \mathbb{Z}$. Recall that there exists a diffeomorphism

$$
\phi_{u}^{-1}\left(C_{u}^{0}\right) \cong C_{u}^{0} \times X_{u}^{0}
$$

over the circle $C_{u}^{0}$ which induces the identity on $X_{u}^{0}$. (See (5.6) or Remark 6.3.) Such a diffeomorphism is unique up to homotopy. By taking the covering of (6.17), we get a diffeomorphism

$$
\phi_{u}^{-1}\left(R_{u}^{0}\right) \cong R_{u}^{0} \times X_{u}^{0}
$$

over $R_{u}^{0}$, which induces the isomorphism (6.1) over each point $a_{u}^{0}\langle\nu\rangle \in R_{u}^{0}$. Let

$$
J\langle\nu\rangle: I \times \dot{\Lambda} \rightarrow \phi_{u}^{-1}\left(R_{u}^{0}\right)
$$

be the composite of the inverse of the diffeomorphism (6.18) with 


$$
\delta_{u}^{0}\langle\nu\rangle \times \text { inclusion }: I \times \Lambda \rightarrow R_{u}^{0} \times X_{u}^{0}
$$

where $\delta_{u}^{0}\langle\nu\rangle: I \rightarrow R_{u}^{0}$ is the lifting of the path $\delta_{u}^{0}$ such that $\delta_{u}^{0}\langle\nu\rangle(0)=a_{u}^{0}\langle\nu\rangle$. Then we have

$$
\partial J\langle\nu\rangle=\Lambda\langle\nu+1\rangle-\Lambda\langle\nu\rangle=\partial(\Gamma\langle\nu+1\rangle-\Gamma\langle\nu\rangle)
$$

Hence

$$
T_{\nu}:=J\langle\nu\rangle-\Gamma\langle\nu+1\rangle+\Gamma\langle\nu\rangle
$$

is an $u^{\text {-cycle in }} F_{u}$. Since $J\langle\nu\rangle$ is contained in $\partial_{0} F_{u}$, we see from Corollary 6.2 that

$$
\left[T_{\nu}\right]=-[\Gamma\langle\nu+1\rangle]+[\Gamma\langle v\rangle]=-\Psi_{u}^{0}\left(\lambda \otimes q^{\nu+1}\right)+\Psi_{u}^{0}\left(\lambda \otimes q^{\nu}\right) \text { in } H_{n}\left(F_{u}, \partial_{0} F_{u}\right)
$$

and hence

$$
\left[T_{\nu}\right]=(1-q) \Psi_{u}^{0}\left(\lambda \otimes q^{\nu}\right) \quad \text { in } \quad H_{n}\left(F_{u}\right)
$$

Note that the $n$-cycle $T_{0}$ in $F_{u}$ is contained in the subspace $\phi_{u}^{-1}\left(K_{01}^{0}\right)$ of $\phi_{u}^{-1}\left(M_{u}^{0}\right)$, where

$$
K_{01}^{0}:=K_{u}\langle 0\rangle \cup \delta_{u}^{0}\langle 0\rangle(I) \cup K_{u}\langle 1\rangle
$$

Consider the composite

$$
\phi_{u}^{-1}\left(M_{u}^{0}\right) \hookrightarrow F_{u} \rightarrow \phi_{u}^{-1}\left(M_{u}^{\infty}\right)
$$

of the inclusion and the retraction (6.3), both of which induce homotopy equivalence. We can choose the maps in (6.19) in such a way that they are lift of the continuous maps on the base space

$$
M_{u}^{0} \hookrightarrow \mathbb{C} \rightarrow M_{u}^{\infty}
$$

which are the inclusion and a retraction. By choosing an appropriate retraction, we can assume that $K_{01}^{0} \subset M_{u}^{0}$ is mapped to

$$
K_{01}^{\infty}:=K_{u}\langle 0\rangle \cup \delta_{u}^{\infty}\langle 0\rangle(I) \cup K_{u}\langle 1\rangle \subset M_{u}^{\infty}
$$


by $(6.20)$, where $\delta_{u}^{\infty}\langle 0\rangle(I)$ is the segment of $R_{u}^{\infty}$ between $a_{u}^{\infty}\langle 0\rangle$ and $a_{u}^{\infty}\langle 1\rangle$. For example, we can choose the retraction $\mathrm{rt}: \mathbb{C} \rightarrow M_{u}^{\infty}$ in such a way that $z-\operatorname{rt}(z) \in \mathbb{R}$ holds for all $z \in \mathbb{C}$. Hence the $n$-cycle $T_{0} \subset \phi_{u}^{-1}\left(K_{01}^{0}\right)$ is mapped by $(6.19)$ to an $n$-cycle $T_{0}^{\prime}$ contained in $\phi_{u}^{-1}\left(K_{01}^{\infty}\right)$. In particular, we have

$$
T_{0}^{\prime} \cap \phi_{u}^{-1}\left(K_{u}\langle\nu\rangle\right)=\emptyset \quad \text { if } \quad \nu \neq 0,1
$$

Hence Corollary 6.3 implies that the homology class $\left[T_{0}\right]=\left[T_{0}^{\prime}\right] \in H_{n}\left(F_{u}\right)$ is written in the form $\Psi_{u}^{\infty}\left(\lambda_{0} \otimes 1+\lambda_{1} \otimes q\right)$ by some $\lambda_{0}, \lambda_{1} \in H_{n-1}\left(X_{u}^{\infty}\right)$.

\section{Description of the Basis of $\mathbb{H}_{n}\left(\mathbb{F}_{u}, \partial_{0} \mathbb{F}_{u}\right)$ and $\mathbb{H}_{n}\left(\mathbb{F}_{u}, \partial_{\infty} \mathbb{F}_{u}\right)$}

In this section, we will describe explicitly $n$-cycles representing the basis of the free $\mathbb{Z}\left[q, q^{-1}\right]$-modules $H_{n}\left(F_{u}, \partial_{0} F_{u}\right)$ and $H_{n}\left(F_{u}, \partial_{\infty} F_{u}\right)$. Throughout this section, we assume $u \in U_{N}^{\tilde{N}}$.

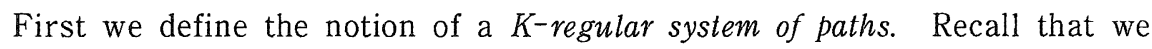
have defined the closed subset $K_{u}$ of $\mathbb{C}^{\times}$for $u \in \mathcal{U}_{N}^{\widetilde{1}}$ in $\S 6$.

Definition 7.1. Suppose that a point $a \in K_{u} \backslash \mathrm{Cr}(u)$ is given. A regular system $\left\{\xi_{1}, \ldots, \xi_{N}\right\}$ of paths from $a$ (see Definition 5.2 ) is said to be $K$-regular if $\xi_{i}(I)$ is contained in $K_{u}$ for $i=1, \ldots, N$.

It is obvious that a $K$-regular system of paths from $a$ always exists for every $u \in \mathcal{U}_{N}$ and every $a \in K_{u} \backslash \operatorname{Cr}(u)$.

Next, we fix some notation concerned with the lifting of objects on $\mathbb{C}^{\times}$and $E_{u}$ by the étale coverings $e: \mathbb{C} \rightarrow \mathbb{C}^{\times}$and $\tilde{e}: F_{u} \rightarrow E_{u}$.

Defingition 7.2. Suppose that a point $\tilde{a} \in \mathbb{C} \backslash \mathscr{C} r(u)$ is given. For $\tilde{p} \in$ $\mathscr{C} r(u)$, let $\mathscr{P}_{u}^{\sim}(\tilde{a}, \tilde{p})$ denote the space of all paths $\omega: I \rightarrow \mathbb{C}$ which satisfy the following: (i) $\omega(0)=\tilde{a}, \omega(1)=\tilde{p}$, and (ii) $\omega([0,1)) \cap \mathscr{C} r(u)=\emptyset$. We equip this space with the compact-open topology, and denote by $\left[\mathscr{P}_{u}^{\sim}(\tilde{a}, \tilde{p})\right]$ the set of path-connected components of $\mathscr{P}_{u}^{\sim}(\tilde{a}, \tilde{p})$. For a path $\omega \in \mathscr{P}_{u}^{\sim}(\tilde{a}, \tilde{p})$, let $[\omega] \in$ $\left[\mathscr{P}_{u}^{\sim}(\tilde{a}, \tilde{p})\right]$ denote the path-connected component of $\mathscr{P}_{u}^{\sim}(\tilde{a}, \tilde{p})$ containing $\omega$; or equivalently, the homotopy class of paths in $\mathscr{P}_{u}^{\sim}(\tilde{a}, \tilde{p})$ represented by $\omega$.

Recall that $(\mathbb{C} \backslash \mathbb{R} \leq 0)\langle\nu\rangle$ is the unique connected component of $e^{-1}(\mathbb{C} \backslash$ $\left.\mathbb{R}_{\leq 0}\right)$ containing $K_{u}\langle\nu\rangle$. For a point $c \in \mathbb{C} \backslash \mathbb{R}_{\leq 0}$, we let $c\langle\nu\rangle$ denote the intersection point of $e^{-1}(c)$ and $\left(\mathbb{C} \backslash \mathbb{R}_{\leq 0}\right)\langle\nu\rangle$. 
Definition 7.3. Suppose that a path $\omega: I \rightarrow \mathbf{C}^{\times}$with $\omega(0) \in \mathbb{C} \backslash \mathbf{R}_{\leq 0}$ is given. Then $\omega\langle\nu\rangle: I \rightarrow \mathbf{C}$ is the unique lifting of $\omega$ to $\mathbf{C}$ by $e: \mathbf{C} \rightarrow \mathbf{C}^{\times}$such that

$$
\omega\langle\nu\rangle(0)=\omega(0)\langle\nu\rangle .
$$

By definition, the following is obvious :

Proposition 7.1. Suppose that $p_{i} \in \operatorname{Cr}(u)$ and $a \in \mathbb{C} \backslash\left(\mathbb{R}_{\leq 0} \cup \operatorname{Cr}(u)\right)$ are given.

(1) For any $\nu, \mu \in \mathbb{Z}$, the map

$$
\left[\mathscr{P}_{u}^{\sim}\left(a\langle\nu\rangle, p_{i}\langle\nu+\mu\rangle\right)\right] \rightarrow\left[\mathscr{P}_{u}\left(a, p_{i}\right)\right]
$$

given by $e: \mathbb{C} \rightarrow \mathbb{C}^{\times}$is injective, and its image is independent of $\nu$. (2) Let $P_{\mu}$ denote the image of (7.1). Then $\left[\mathscr{P}_{u}\left(a, p_{\imath}\right)\right]$ is the disjoint union of all $P_{\mu}(\mu \in \mathbb{Z})$. (3) The homotopy class $[\omega] \in\left[\mathscr{P}_{u}\left(a, p_{i}\right)\right]$ is contained in $P_{\mu}$ if and only if $(\omega\langle\nu\rangle)(1)=(\omega(1))\langle\nu+\mu\rangle$ for all $\nu \in \mathbb{Z}$. In particular, if $\omega(I) \subset \mathbb{C} \backslash \mathbb{R}_{\leq 0}$, then $[\omega] \in P_{0}$.

Definition 7.4. Suppose that a path $\omega \in \mathscr{P}_{u}\left(a, p_{i}\right)$ with $a \in \mathbf{C} \backslash\left(\mathbf{R}_{\leq 0} \cup\right.$ $\mathrm{Cr}(u))$ and an integer $\nu \in \mathbb{Z}$ are given. Then the homotopy class $[\omega\langle\nu\rangle]$, which is an element of $\left[\mathscr{P}_{u}^{\sim}\left(a\langle\nu\rangle, p_{i}\langle\nu+\mu\rangle\right]\right.$ with $p_{i}\langle\nu+\mu\rangle=\omega\langle\nu\rangle$ (1) for some $\mu \in \mathbb{Z}$, is uniquely determined by the homotopy class $[\omega]$; that is, $\left[\omega^{\prime}\right.$ $\langle\nu\rangle]=[\omega\langle\nu\rangle]$ holds for all $\omega^{\prime} \in[\omega]$. Hence we can denote $[\omega\langle\nu\rangle]$ by $[\omega]\langle\nu\rangle$.

For a path $\omega \in \mathscr{P}_{u}\left(a, p_{\imath}\right)$ with $a \in \mathbb{C} \backslash(\mathbb{R} \leq 0 \cup \operatorname{Cr}(u))$, we have a vanishing cycle

$$
\sigma[\omega] \in\left[S^{n-1}, \phi_{u}^{-1}(a)\right]
$$

for $[\omega]$, unique up to sign, and the thimble

$$
\theta([\omega], \sigma[\omega]) \in\left[\left(C S^{n-1}, S^{n-1}\right),\left(E_{u}, \phi_{u}^{-1}(a)\right)\right]
$$

for $[\omega]$ starting from $\sigma[\omega]$.

Definition 7.5. For each $\nu \in \mathbb{Z}$, the vanishing cycle $\sigma[\omega]$ lifts uniquely to a vanishing cycle

$$
\sigma[\omega]\langle\nu\rangle \in\left[S^{n-1}, \phi_{u}^{-1}(a\langle\nu\rangle)\right]
$$


which is one of the two vanishing cycles for $[\omega]\langle\nu\rangle=[\omega\langle\nu\rangle] \in\left[\mathscr{P}_{u}^{\sim}(a\langle\nu\rangle\right.$, $\left.\left.p_{i}\langle\nu+\mu\rangle\right)\right]$. Also the thimble $\theta([\omega], \sigma[\omega])$ lifts uniquely to the thimble

$$
\theta([\omega\langle\nu\rangle], \sigma[\omega]\langle\nu\rangle) \in\left[\left(C S^{n-1}, S^{n-1}\right),\left(F_{u}, \psi_{u}^{-1}(a\langle\nu\rangle)\right)\right]
$$

for $[\omega]\langle\nu\rangle=[\omega\langle\nu\rangle]$ starting from $\sigma[\omega]\langle\nu\rangle$. The thimble $\theta([\omega\langle\nu\rangle], \sigma[\omega]$ $\langle\nu\rangle)$ is uniquely determined by $[\omega] \in\left[\mathscr{P}_{u}\left(a, p_{i}\right)\right], \nu \in \mathbb{Z}$, and the choice of the sign of $\sigma[\omega]$. Hence we can use the following notation for denoting the lifted thimble :

$$
\theta([\omega], \sigma[\omega])\langle\nu\rangle:=\theta([\omega\langle\nu\rangle], \sigma[\omega]\langle\nu\rangle)
$$

Its homology class is denoted by

$$
\bar{\theta}([\omega], \sigma[\omega])\langle\nu\rangle \in H_{n}\left(F_{u}, \psi_{u}^{-1}(a\langle\nu\rangle)\right)
$$

As before, when the orientation is irrelevant, we write them simply by $\theta([\omega])$ $\langle\nu\rangle$ and $\bar{\theta}([\omega])\langle\nu\rangle$.

When $a=a_{u}^{0}$ (resp. $a=a_{u}^{\infty}$ ), this homology class can be considered as an element of $H_{n}\left(F_{u}, \partial_{0} F_{u}\right)$ (resp. of $H_{n}\left(F_{u}, \partial_{\infty} F_{u}\right)$ ), which will be denoted by the same symbol $\bar{\theta}([\omega], \sigma[\omega])\langle\nu\rangle$. By definition, we have

$$
q \bar{\theta}([\omega], \sigma[\omega])\langle\nu\rangle=\bar{\theta}([\omega], \sigma[\omega])\langle\nu+1\rangle
$$

in the $\mathbb{Z}\left[q, q^{-1}\right]$-module $H_{n}\left(F_{u}, \partial_{0} F_{u}\right)\left(\right.$ resp. $\left.H_{n}\left(F_{u}, \partial_{\infty} F_{u}\right)\right)$.

Proposition 7.2. Suppose that $u \in \mathcal{U}_{N}^{\tilde{N}}$. Let $p_{i}$ be a value in $\mathrm{Cr}(u)$, and let $\omega$ be an element of $\mathscr{P}_{u}\left(a_{u}^{0}, p_{i}\right)$ (resp. of $\left.\mathscr{P}_{u}\left(a_{u}^{\infty}, p_{i}\right)\right)$ such that $\omega(I) \subset K_{u}$. Then we have

$$
\begin{aligned}
\bar{\theta}([\omega], \sigma[\omega])\langle\nu\rangle & =-\Psi_{u}^{0}\left(\bar{\sigma}[\omega] \otimes q^{\nu}\right) \\
& \left(\text { resp. } \bar{\theta}([\omega], \sigma[\omega])\langle\nu\rangle=-\Psi_{u}^{\infty}\left(\bar{\sigma}[\omega] \otimes q^{\nu}\right)\right)
\end{aligned}
$$

holds in $H_{n}\left(F_{u}, \partial_{0} F_{u}\right) \quad\left(\right.$ resp. in $\left.H_{n}\left(F_{u}, \partial_{\infty} F_{u}\right)\right)$

Proof. Let $\Gamma: C S^{n-1} \rightarrow E_{u}$ be a continuous map representing the thimble $\theta$ $([\omega], \sigma[\omega])$ over the path $\omega$. Since $\omega(I) \subset K_{u}$, the $n$-chain $\Gamma$ is contained in $\phi_{u}^{-1}\left(K_{u}\right)$. Its boundary $\partial \Gamma$ represents $-\bar{\sigma}[\omega]$ in $H_{n-1}\left(X_{u}^{0}\right)$ (resp. in $H_{n-1}$ $\left.\left(X_{u}^{\infty}\right)\right)$ by the anti-commutativity of $(4.1)$. The homology class $\bar{\theta}([\omega], \sigma[\omega])$ $\langle\nu\rangle$ is represented by the $n$-chain $\Gamma\langle\nu\rangle \subset \phi_{u}^{-1}\left(K_{u}\langle\nu\rangle\right)$ corresponding to $\Gamma$ via 
the isomorphism (6.6). Hence Corollary 6.2 implies (7.3).

Proposition 7.3. Suppose that $u \in \mathcal{U}_{N}^{\sim}$.

(0) Let $\left\{\xi_{1}^{0}, \ldots, \xi_{N}^{0}\right\}$ be a K-regular system of paths from $a_{u}^{0}$, and let $\sigma\left[\xi_{1}^{0}\right] \in$ $\left[S^{n-1}, X_{u}^{0}\right]$ be a vanishing cycle for $\left[\xi_{i}^{0}\right]$. Then the homology classes

$$
\bar{\theta}\left(\left[\xi_{1}^{0}\right], \sigma\left[\xi_{1}^{0}\right]\right)\langle 0\rangle, \ldots \ldots \ldots, \bar{\theta}\left(\left[\xi_{N}^{0}\right], \sigma\left[\xi_{N}^{0}\right]\right)\langle 0\rangle
$$

of the lifted thimbles form a set of basis for the free $\mathbb{Z}\left[q, q^{-1}\right]$-module $H_{n}\left(F_{u}, \partial_{0} F_{u}\right)$.

$(\infty)$ Let $\left\{\xi_{1}^{\infty}, \ldots, \xi_{N}^{\infty}\right\}$ be a $K^{-r e g u l a r ~ s y s t e m ~ o f ~ p a t h s ~ f r o m ~} a_{u}^{\infty}$, and let $\sigma\left[\xi_{1}^{\infty}\right] \in$ $\left[S^{n-1}, X_{u}^{\infty}\right]$ be a vanishing cycle for $\left[\xi_{i}^{\infty}\right]$. Then the homology classes

$$
\bar{\theta}\left(\left[\xi_{1}^{\infty}\right], \sigma\left[\xi_{1}^{\infty}\right]\right)\langle 0\rangle, \ldots \ldots \ldots, \bar{\theta}\left(\left[\xi_{N}^{\infty}\right], \sigma\left[\xi_{N}^{\infty}\right]\right)\langle 0\rangle
$$

of the lifted thimbles form a set of basis for the frec $\mathbb{Z}\left[q, q^{-1}\right]$-module $H_{n}\left(F_{u}, \partial_{\infty} F_{u}\right)$.

Proof. By the assumption of $K$-regularity. Proposition 5.1 implies that $\bar{\sigma}\left[\xi_{1}^{0}\right], \ldots, \bar{\sigma}\left[\xi_{N}^{0}\right]$ form a set of basis for the free $\mathbb{Z}$-module $H_{n-1}\left(X_{u}^{0}\right)$, and Proposition 7.2 implies that

$$
\bar{\theta}\left(\left[\xi_{i}^{0}\right], \sigma\left[\xi_{i}^{0}\right]\right)\langle\nu\rangle=-\Psi_{u}^{0}\left(\bar{\sigma}\left[\xi_{\imath}^{0}\right] \otimes q^{\nu}\right)
$$

Hence the assertion (0) follows Theorem 6.1 and (7.2). The assertion $(\infty)$ follows from

$$
\bar{\theta}\left(\left[\xi_{1}^{\infty}\right], \sigma\left[\xi_{1}^{\infty}\right]\right)\langle\nu\rangle=-\Psi_{u}^{\infty}\left(\bar{\sigma}\left[\xi_{1}^{\infty}\right] \otimes q^{\nu}\right)
$$

by the same argument.

Theorem 7.1. Let $b \in \mathcal{U}$ be a base point which is contained in $\mathcal{U}_{N}^{\tilde{N}}$. The homomorphism

$$
H_{n}\left(F_{b}, \partial_{0} F_{b}\right) \rightarrow H_{n-1}\left(X_{b}^{0}\right)
$$

which is the composite of the inverse map of $\Psi_{b}^{0}$ and the homomorphism $H_{n-1}\left(X_{b}^{0}\right)$ $\otimes \mathbb{Z}\left[q, q^{-1}\right] \rightarrow H_{n-1}\left(X_{b}^{0}\right)$ given by $q \mapsto 1$ is $\pi_{1}(\mathcal{U}, b)$-equivariant.

Proof. We will prove this theorem by showing that (7.5) is equal with the composite 


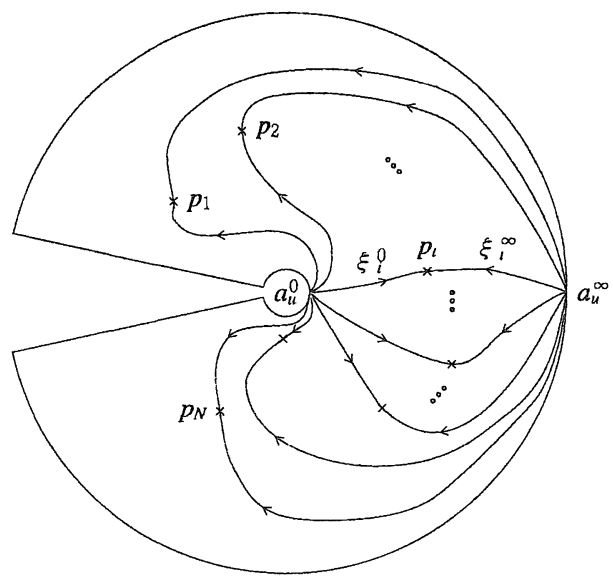

$K$-regular systems of paths $\left\{\xi_{1}^{0} \ldots, \xi_{N}^{0}\right\}$ from $a_{u}^{0}$ and $\left\{\xi_{1}^{\infty}, \ldots, \xi_{N}^{\infty}\right\}$ from $a_{u}^{\infty}$

Figure 6

$$
H_{n}\left(F_{b}, \partial_{0} F_{b}\right) \underset{\tilde{e_{*}}}{\longrightarrow} H_{n}\left(E_{b}, \partial_{0} E_{b}\right) \underset{(\mathrm{A})}{\stackrel{\sim}{\longrightarrow}} H_{n-1}\left(X_{b}^{0}\right),
$$

where $\tilde{e}_{*}$ is the homomorphism induced from the covering map $\tilde{e}: F_{b} \rightarrow E_{b}$ and (A) is the isomorphism in Proposition 5.2(6). It is obvious that $\tilde{e}_{*}$ is $\pi_{1}(U, b)$ equivariant. By Proposition 5.2(7),(A) is also $\pi_{1}(\mathcal{U}, b)$-equivariant.

We fix a $K$-regular system $\left\{\xi_{1}^{0}, \ldots, \xi_{N}^{0}\right\}$ of paths from $a_{b}^{0}$ and, for each $i=$ $1, \ldots, N$, we choose a vanishing cycle $\sigma\left[\xi_{i}^{0}\right] \in\left[S^{n-1}, X_{b}^{0}\right]$. We put

$$
\bar{\theta}_{i}^{0}\langle\nu\rangle:=\bar{\theta}\left(\left[\xi_{i}^{0}\right], \sigma\left[\xi_{i}^{0}\right]\right)\langle\nu\rangle \in H_{n}\left(F_{b}, \partial_{0} F_{b}\right) .
$$

We have $\bar{\theta}_{i}^{0}\langle\nu\rangle=q^{\nu} \bar{\theta}_{i}^{0}\langle 0\rangle$ for all $\nu \in \mathbb{Z}$ by (7.2). By Proposition 7.3, the set

$$
\left\{\bar{\theta}_{i}^{0}\langle\nu\rangle ; \nu \in \mathbb{Z} \text { and } i=1, \ldots, N\right\}
$$

form a set of basis for the free $\mathbb{Z}$-module $H_{n}\left(F_{b}, \partial_{0} F_{b}\right)$. Therefore, it is enough to show that the two homomorphisms (7.5) and (7.6) map each $\bar{\theta}_{i}^{0}\langle\nu\rangle$ to a same element of $H_{n-1}\left(X_{b}^{0}\right)$.

By (7.4), the homomorphism (7.5) maps $\bar{\theta}_{i}^{0}\langle\nu\rangle$ to $-\bar{\sigma}\left[\xi_{i}^{0}\right]$. On the 
other hand, $\tilde{e}_{*}$ maps to $\bar{\theta}_{i}^{0}\langle\nu\rangle$ to $\bar{\theta}\left(\left[\xi_{l}^{0}\right], \sigma\left[\xi_{i}^{0}\right]\right) \in H_{n}\left(E_{b}, \partial_{0} E_{b}\right)$ because of the definition of the lifting. By the isomorphism (5.8), this element is mapped to

$$
\partial \bar{\theta}\left(\left[\xi_{i}^{0}\right], \sigma\left[\xi_{i}^{0}\right]\right)=-\bar{\sigma}\left[\xi_{i}^{0}\right]
$$

because of the anti-commutativity of (4.1). Hence (7.6) also maps $\theta_{i}^{0}\langle\nu\rangle$ to $\bar{\sigma}\left[\xi_{1}^{0}\right] \in H_{n-1}\left(X_{b}^{0}\right)$.

Remark 7.1. The isomorphism (0.3) in Introduction is obtained as follows:

$$
H_{n}\left(F_{b}\right) \underset{(\mathrm{B})}{\stackrel{\sim}{\rightarrow}} H_{n}\left(F_{b}, \partial_{0} F_{b}\right) \underset{\left(\Psi_{b}^{0}\right)^{-1}}{\stackrel{\sim}{\longrightarrow}} H_{n-1}\left(X_{b}^{0}\right) \otimes_{\mathbb{Z}} \mathbb{Z}\left[q, q^{-1}\right] \underset{(\mathrm{C})}{\stackrel{\sim}{\longrightarrow}} H_{n-1}\left(X_{b}\right) \otimes_{\mathbb{Z}} \mathbb{Z}\left[q, q^{-1}\right] .
$$

where (B) is the multiplication by $1 /(1-q)$, and (C) is the isomorphism $H_{n-1}$ $\left(X_{b}^{0}\right) \cong H_{n-1}\left(X_{b}\right)$ induced from (5.1) tensored with the identity on $\mathbb{Z}\left[q, q^{-1}\right]$. Then, by Lemmas $1.2,5.1$ and Theorem 7.1 , we see that $(0.3)$ has the required property.

88. Intersection Forms on $\mathbb{H}_{n}\left(\mathbb{F}_{u}, \partial_{0} F_{u}\right) \times \mathbb{H}_{n}\left(\boldsymbol{F}_{u}, \partial_{\infty} F_{u}\right)$

As in [6], we introduce "hermitian" intersection forms

$$
\begin{array}{ll}
(,)_{0}: H_{n}\left(F_{u}, \partial_{\infty} F_{u}\right) \times H_{n}\left(F_{u}, \partial_{0} F_{u}\right) \rightarrow \mathbb{Z}\left[q, q^{-1}\right], \quad \text { and } \\
(,)_{\infty}: H_{n}\left(F_{u}, \partial_{0} F_{u}\right) \times H_{n}\left(F_{u}, \partial_{\infty} F_{u}\right) \rightarrow \mathbb{Z}\left[q, q^{-1}\right],
\end{array}
$$

for $u \in \mathcal{U}$. Note that the usual intersection form

$$
\langle,\rangle: H_{n}\left(F_{u}, \partial_{\infty} F_{u}\right) \times H_{n}\left(F_{u}, \partial_{0} F_{u}\right) \rightarrow \mathbb{Z}
$$

is well-defined. (See §3.) For $x \in H_{n}\left(F_{u}, \partial_{\infty} F_{u}\right)$ and $y \in H_{n}\left(F_{u}, \partial_{0} F_{u}\right)$, we put

$$
(x, y)_{0}:=\sum_{\nu \in \mathbb{Z}}\left\langle x, q^{\nu} y\right\rangle q^{\nu} \in \mathbb{Z}\left[q, q^{-1}\right] .
$$

Let $*: \mathbb{Z}\left[q, q^{-1}\right] \rightarrow \mathbb{Z}\left[q, q^{-1}\right]$ be the ring automorphism given by $* q=q^{-1}$. It is obvious that $\left\langle q^{\nu} x, q^{\nu} y\right\rangle=\langle x, y\rangle$ for all $\nu \in \mathbb{Z}$. Therefore, for arbitrary $a, a^{\prime}, b$, $b^{\prime} \in \mathbb{Z}\left[q, q^{-1}\right]$, we have, 


$$
\begin{aligned}
& \left(a x+a^{\prime} x^{\prime}, y\right)_{0}=a(x, y)_{0}+a^{\prime}\left(x^{\prime}, y\right)_{0}, \quad \text { and } \\
& \left(x, b y+b^{\prime} y^{\prime}\right)_{0}=* b(x, y)_{0}+* b^{\prime}\left(x, y^{\prime}\right)_{0} .
\end{aligned}
$$

We define the hermitian form $(,)_{\infty}$ by

$$
(x, y)_{\infty}:=*(y, x)_{0}
$$

Remark 8.1. For any $[\gamma] \in \pi_{1}(\mathcal{u}, b)$, we have $\left\langle[\gamma]_{* x},[\gamma]_{* y}\right\rangle=\langle x, y\rangle$. Combining this with Lemma 1.2 , we get

$$
\left([\gamma]_{*} x,[\gamma]_{* y}\right)_{0}=(x, y)_{0}
$$

This implies that $(,)_{0}$ and $(,)_{\infty}$ are hermitian intersectian forms between the locally constant systems on $\mathcal{U}$ corresponding to $H_{n}\left(F_{b}, \partial_{0} F_{b}\right)$ and $H_{n}\left(F_{b}, \partial_{\infty} F_{b}\right)$.

Lemma 8.1. Suppose that $u \in U_{N}^{\sim}$. Let $\alpha$ and $\beta$ be elements of $H_{n-1}\left(X_{u}^{\infty}\right)$ and $H_{n-1}\left(X_{u}^{0}\right)$, respectively. Then the integer $\left\langle\Psi_{u}^{\infty}\left(\alpha \otimes q^{\nu}\right), \Psi_{u}^{0}\left(\beta \otimes q^{u}\right)\right\rangle$ is zero unless $\nu=\mu$.

Proof. By Corollary 6.2 and Remark 6.4, $\Psi_{u}^{\infty}\left(\alpha \otimes \cdot q^{\nu}\right)$ is represented by an $n$-chain $\Gamma_{\alpha}\langle\nu\rangle$ contained in $\phi_{u}^{-1}\left(K_{u}\langle\nu\rangle\right)$, while $\Psi_{u}^{0}\left(\beta \otimes q^{\mu}\right)$ is represented by an $n$-chain $\Gamma_{\beta}\langle\mu\rangle$ contained in $\psi_{u}^{-1}\left(K_{u}\langle\mu\rangle\right)$. If $\nu \neq \mu$, then $K_{u}\langle\nu\rangle \cap K_{u}\langle\mu\rangle=\emptyset$, and hence $\left\langle\left[\Gamma_{\alpha}\langle\nu\rangle\right],\left[\Gamma_{\beta}\langle\mu\rangle\right]\right\rangle=0$.

Combining Lemma 8.1 with (8.1), we get the following formula. Let $\alpha_{\nu}(\nu \in \mathbb{Z})$ and $\beta_{\mu}(\mu \in \mathbb{Z})$ be elements of $H_{n-1}\left(X_{u}^{\infty}\right)$ and $H_{n-1}\left(X_{u}^{0}\right)$, respectively, such that almost all of them are zero. Then

$$
\begin{gathered}
\left(\Psi_{u}^{\infty}\left(\sum_{\nu \in \mathbb{Z}} \alpha_{\nu} \otimes q^{\nu}\right), \quad \Psi_{u}^{0}\left(\sum_{\mu \in \mathbb{Z}} \beta_{\mu} \otimes q^{\mu}\right)\right)_{0} \\
=\sum_{k \in \mathbb{Z}}\left(\sum_{\nu-\mu=k}\left\langle\Psi_{u}^{\infty}\left(\alpha_{\nu} \otimes 1\right), \quad \Psi_{u}^{0}\left(\beta_{\mu} \otimes 1\right)\right\rangle\right) \cdot q^{k} .
\end{gathered}
$$

Lemma 8.2. Suppose that $u \in \mathcal{U}_{N}^{\tilde{N}}$. Let $p$ and $p^{\prime}$ be values in $\mathrm{Cr}(u)$, and let $\xi^{0}$ and $\xi^{\infty}$ be paths in $\mathscr{P}_{u}\left(a_{u}^{0}, p\right)$ and $\mathscr{P}_{u}\left(a_{u}^{\infty}, p^{\prime}\right)$, respectively. Suppose that $\xi^{0}(I) \subset K_{u}$ and $\xi^{\infty}(I) \subset K_{u}$. (1) Suppose that $p=p^{\prime}$ and $\xi^{0}(I) \cap \xi^{\infty}(I)=\{p\}$. Then

$$
\left(\bar{\theta}\left(\left[\xi^{\infty}\right]\right)\langle\nu\rangle, \bar{\theta}\left(\left[\xi^{0}\right]\right)\langle\mu\rangle\right)_{0}= \pm q^{\nu-\mu} .
$$


(2) Suppose that $p \neq p^{\prime}$ and $\xi^{0}(I) \cap \xi^{\infty}(I)=\emptyset$. Then

$$
\left(\bar{\theta}\left(\left[\xi^{\infty}\right]\right)\langle\nu\rangle, \bar{\theta}\left(\left[\xi^{0}\right]\right)\langle\mu\rangle\right)_{0}=0 .
$$

Proof. By (8.2) and Proposition 7.2, we see that $\left(\bar{\theta}\left(\left[\xi^{\infty}\right]\right)\langle\nu\rangle, \bar{\theta}\left(\left[\xi^{0}\right]\right)\right.$ $\langle\mu\rangle)_{0}$ is a multiple of $q^{\nu-\mu}$ by the integer $\left\langle\bar{\theta}\left(\left[\xi^{\infty}\right]\right)\langle 0\rangle, \bar{\theta}\left(\left[\xi^{0}\right]\right)\langle 0\rangle\right\rangle$. Let $T^{0}$ : $C S^{n-1} \rightarrow F_{u}$ and $T^{\infty}: C S^{n-1} \rightarrow F_{u}$ be continuous maps representing the thimble $\theta\left(\left[\xi^{0}\right]\right)\langle 0\rangle$ over $\xi^{0}\langle 0\rangle$, and the thimble $\theta\left(\left[\xi^{\infty}\right]\right)\langle 0\rangle$ over $\xi^{\infty}\langle 0\rangle$, respectively. By the assumptions on the paths $\xi^{0}$ and $\xi^{\infty}$, we have

$$
\xi^{0}\langle 0\rangle(I) \cap \xi^{\infty}\langle 0\rangle(I)=\left\{\begin{array}{l}
\{p\langle 0\rangle\} \text { in the case (1), and } \\
\emptyset \text { in the case (2). }
\end{array}\right.
$$

In the case (1), using Lemma 4.1, we can choose the $n$-chains $T^{0}$ and $T^{\infty}$ in such a way that they intersect only at the critical point of $\phi_{u}$ over $p\langle 0\rangle$, and that the intersection is transverse. Hence $\left\langle\left[T^{\infty}\right],\left[T^{0}\right]\right\rangle=\left\langle\bar{\theta}\left(\left[\xi^{\infty}\right]\right)\langle 0\rangle\right.$,

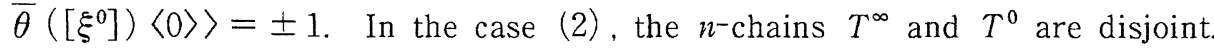
Hence $\left\langle\left[T^{\infty}\right],\left[T^{0}\right]\right\rangle$ is zero.

Now we shall prove the following:

Proposition 8.1. The intersection forms $(,)_{0}$ and $(,)_{\infty}$ are non-degenerate.

Here the non-degeneracy of $(,)_{0}$ means that the map

$$
H_{n}\left(F_{u}, \partial_{0} F_{u}\right) \rightarrow \operatorname{Hom}_{\mathbf{Z}\left\{q, q^{-1}\right]}\left(H_{n}\left(F_{u}, \partial_{\infty} F_{u}\right), \quad \mathbb{Z}\left[q, q^{-1}\right]\right)
$$

given by $y \mapsto(, y)_{0}$ is a bijection.

Proof. By Remark 8.1, it is enough to prove Proposition 8.1 under the assumption that $u \in \mathcal{U}_{N}^{\tilde{N}}$. We can take $K$-regular systems $\left\{\xi_{1}^{0}, \ldots, \xi_{N}^{0}\right\}$ and $\left\{\xi_{1}^{\infty}, \ldots, \xi_{N}^{\infty}\right\}$ of paths from $a_{u}^{0}$ and from $a_{u}^{\infty}$, respectively, in such a way that the following holds :

$$
\xi_{i}^{0}(I) \cap \xi_{j}^{\infty}(I)= \begin{cases}\emptyset \quad \text { if } i \neq j, \text { and } \\ \left\{p_{\imath}\right\} \text { if } i=j .\end{cases}
$$

By Lemma 8.2, we have

$$
\left.\left.\left(\bar{\theta}\left[\xi_{i}^{\infty}\right]\right)\langle 0\rangle, \bar{\theta}\left(\left[\xi_{j}^{0}\right]\right)\langle 0\rangle\right)_{0}=\left\langle\bar{\theta}\left(\left[\xi_{i}^{\infty}\right]\right)\langle 0\rangle, \bar{\theta}\left(\left[\xi_{j}^{0}\right]\right)\langle 0\rangle\right)\right\rangle= \pm \delta_{i j}
$$


Thus, in terms of the basis $\left\{\bar{\theta}\left(\left[\xi_{i}^{0}\right]\right)\langle 0\rangle ; i=1, \ldots, N\right\}$ of $H_{n}\left(F_{u}, \partial_{0} F_{u}\right)$ over $\mathbb{Z}[q$, $\left.q^{-1}\right]$ and $\left\{\bar{\theta}\left(\left[\xi_{i}^{\infty}\right]\right)\langle 0\rangle ; i=1, \ldots, N\right\}$ of $H_{n}\left(F_{u}, \partial_{\infty} F_{u}\right)$ over $\mathbb{Z}\left[q, q^{-1}\right]$, the intersection form $(,)_{0}$ is expressed by a diagonal matrix with diagonal coefficients \pm 1 .

Definition 8.1. An element $x \in H_{n}\left(F_{u}, \partial_{0} F_{u}\right)$ (resp. $y \in H_{n}\left(F_{u}, \partial_{\infty} F_{u}\right)$ ) is called primitive if there exists an element $x^{\prime} \in H_{n}\left(F_{u}, \partial_{\infty} F_{u}\right)$ (resp. $y^{\prime} \in H_{n}\left(F_{u}\right.$, $\left.\left.\partial_{0} F_{u}\right)\right)$ such that $\left(x^{\prime}, x\right)_{0}=1\left(\operatorname{resp} .\left(y^{\prime}, y\right)_{\infty}=1\right)$.

$\mathbb{D e f i n i m i t i o n ~ 8 . 2 . ~ L e t ~} U\left(\mathbb{Z}\left[q, q^{-1}\right]\right)$ denote the group of the units $\left\{ \pm q^{\nu} ; \nu \in\right.$ $\mathbb{Z}$ \} of the ring $\mathbb{Z}\left[q, q^{-1}\right]$. We say that two elements $x$ and $x^{\prime}$ of a $\mathbb{Z}\left[q, q^{-1}\right]$ -module is said to be congruent modulo $U\left(\mathbb{Z}\left[q, q^{-1}\right]\right)$ and write $x \equiv x^{\prime}$, if there exists a unit $a \in U\left(\mathbb{Z}\left[q, q^{-1}\right]\right)$ such that $x=a x^{\prime}$.

For example if $x$ is a primitive element of $H_{n}\left(F_{u}, \partial_{\infty} F_{u}\right)$ and $x \equiv x^{\prime}$, then $x^{\prime}$ is also primitive.

\section{\$2. Pieard-Lefschetz Formula for Local Monodromies around $\mathscr{D}_{0}$ and $\mathscr{D}_{\infty}$}

\section{\$2.1. Definition of Simple Loops and Local Monodromies}

We fix a base point $b \in \mathcal{U}$.

Definition 9.1.1. A loop $\gamma ; I \rightarrow \mathcal{U}$ with the base point $b$ is called a simple loop around $\mathscr{D}_{0}\left(\right.$ resp. $\left.\mathscr{D}_{\infty}\right)$ if the following are satisfied; (i) there exist a non-singular point $c$ on $\mathscr{D}_{0} \backslash\left(\mathscr{D}_{0} \cap \mathscr{D}_{\infty}\right)\left(\right.$ resp. $\left.\mathscr{D}_{\infty} \backslash\left(\mathscr{D}_{0} \cap \mathscr{D}_{\infty}\right)\right)$ and a small closed disk $\Delta$ in $\Gamma$ with the center $c$ which intersects $\mathscr{D}_{0} \cup \mathscr{D}_{\infty}$ transversely at only one point $c$, (ii) there exists a path $\beta$ on $U$ from $b$ to a point $b^{\prime}$ on the boundary $\partial \Delta$ of $\Delta$, and (iii) the loop $\gamma$ starts from $b$, goes to $b^{\prime}$ along $\beta$, draws a circle $\partial \Delta$ in the counter-clockwise direction, and goes back to $b$ along $\beta^{-1}$.

Definition 9.1.2. Let $\gamma: I \rightarrow \mathcal{U}$ be a simple loop around $\mathscr{D}_{0}$ (resp. $\mathscr{D}_{\infty}$ ). Then the monodromy action $[\gamma] *$ on various sets or groups is called a local monodromy around $\mathscr{D}_{0}\left(\right.$ resp. $\left.\mathscr{D}_{\infty}\right)$.

Proposition 9.1.1. Let $b$ and $b^{\prime \prime}$ be two base points of $U$, and let $\gamma: I \rightarrow U$ and $\gamma^{\prime}: I \rightarrow \mathcal{U}$ be simple loops around $\mathscr{D}_{0}$ with the base points $b$ and $b^{\prime}$, respectively. Then there exists a path $\alpha: I \rightarrow \mathcal{U}$ from $b$ to $b^{\prime}$ such that $\left[\alpha^{-1} \gamma^{\prime} \alpha\right]=[\gamma]$ in 
$\pi_{1}(\mathcal{U}, b)$. The same assertion holds for simple loops around $\mathscr{D}_{\infty}$.

Proof. Since both of the hypersurfaces $\mathscr{D}_{0}$ and $\mathscr{D}_{\infty}$ are irreducible, each of the non-singular loci of $\mathscr{D}_{0} \backslash\left(\mathscr{D}_{0} \cap \mathscr{D}_{\infty}\right)$ and $\mathscr{D}_{\infty} \backslash\left(\mathscr{D}_{0} \cap \mathscr{D}_{\infty}\right)$ is also irreducible.

\section{\$9.2. Picard-Lefschetz Formula}

Now we shall state our main theorems.

Theorem 9.2.1. Let $\left[\gamma_{0}\right] \in \pi_{1}(\mathcal{U}, b)$ be the homotopy class of a simple loop around $\mathscr{D}_{0}$. There exists a pair

$$
\left(v\left[\gamma_{0}\right], v^{\sim}\left[\gamma_{0}\right]\right) \in H_{n}\left(F_{b}\right) \times H_{n}\left(F_{b}, \partial_{0} F_{b}\right)
$$

such that the local monodromy $\left[\gamma_{0}\right]_{*}$ around $\mathscr{D}_{0}$ on $H_{n}\left(F_{b}, \partial_{\infty} F_{b}\right)$ is given by

$$
x \mapsto x+\left(x, v^{\sim}\left[\gamma_{0}\right]\right)_{0} \cdot v\left[\gamma_{0}\right]
$$

Moreover, such a pair $\left(v\left[\gamma_{0}\right], v^{\sim}\left[\gamma_{0}\right]\right)$ is unique up to $U\left(\mathbb{Z}\left[q, q^{-1}\right]\right)$, and $v^{\sim}\left[\gamma_{0}\right]$ is primitive. We also have

$$
v\left[\gamma_{0}\right]=(-1)^{n(n-1) / 2}(q-1) \cdot v^{2}\left[\gamma_{0}\right]
$$

Theorem 9.2.2. Let $\left[\gamma_{\infty}\right] \in \pi_{1}(\mathcal{U}, b)$ be the homotopy class of a simple loop around $\mathscr{D}_{\infty}$. There exists a pair

$$
\left(v\left[\gamma_{\infty}\right], v^{\sim}\left[\gamma_{\infty}\right]\right) \in H_{n}\left(F_{b}, \partial_{0} F_{b}\right) \times H_{n}\left(F_{b}, \partial_{\infty} F_{b}\right)
$$

such that the local monodromy $\left[\gamma_{\infty}\right]_{*}$ around $\mathscr{D}_{\infty}$ on $H_{n}\left(F_{b}, \partial_{0} F_{b}\right)$ is given by

$$
x \mapsto x+\left(x, v^{2}\left[\gamma_{\infty}\right]\right)_{\infty} \cdot v\left[\gamma_{\infty}\right]
$$

Moreover, such a pair $\left(v\left[\gamma_{\infty}\right], v^{\sim}\left[\gamma_{\infty}\right]\right)$ is unique up to $U\left(\mathbb{Z}\left[q, q^{-1}\right]\right)$, and $v^{\sim}\left[\gamma_{\infty}\right]$ is primitive.

Remark 9.2.1. Comparing Theorems 9.2.1 and 9.2.2, we can see that there is a certain kind of duality between " 0 " and " $\infty$ ". This duality, however, is not perfect. Contrary to the case in Theorem 9.2.1, the homology class $v\left[\gamma_{\infty}\right] \in$ $H_{n}\left(F_{b}, \partial_{0} F_{b}\right)$ in Theorem 9.2 .2 is not contained in $H_{n}\left(F_{b}\right)$. This difference comes from the fact that,while the action of $\left[\gamma_{0}\right]_{*}$ on $H_{n-1}\left(X_{b}^{\infty}\right)$ is trivial (cf. 
Claim 2 in the proof of Proposition 9.4.1 below), the action of $\left[\gamma_{\infty}\right]_{*}$ on $H_{n-1}\left(X_{b}^{0}\right)$ is non-trivial (cf. Proposition 9.7.1). Moreover, the relation between $v^{\sim}\left[\gamma_{\infty}\right]$ and $v\left[\gamma_{\infty}\right]$ is not so simple as (9.2.2). A detailed description of $v\left[\gamma_{\infty}\right]$ is given in Proposition 9.7.2.

Remark 9.2.2. The uniqueness of $\left(v\left[\gamma_{0}\right], v^{\vee}\left[\gamma_{0}\right]\right)$ in Theorems 9.2 .1 follows easily from the property $(9.2 .1)$ and the primitiveness of $v^{\sim}\left[\gamma_{0}\right]$. Suppose that (9.2.1) holds for all $x \in H_{n}\left(F_{b}, \partial_{\infty} F_{b}\right)$ with some pair $\left(v\left[\gamma_{0}\right], v^{\sim}\left[\gamma_{0}\right]\right)$, and that $v^{v}\left[\gamma_{0}\right]$ is primitive. Then the image of the endomorphism Id $-\left[\gamma_{0}\right]_{*}$ of $H_{n}\left(F_{b}\right.$, $\left.\partial_{\infty} F_{b}\right)$ is a free $\mathbb{Z}\left[q, q^{-1}\right]$-module of rank 1 , and hence its generator $v\left[\gamma_{0}\right]$ is determined uniquely modulo $U\left(\mathbb{Z}\left[q, q^{-1}\right]\right)$. Suppose that a generator $v\left[\gamma_{0}\right]$ is fixed. Then the endomorphism Id $-\left[\gamma_{0}\right]_{*}$ is written in the form $x \mapsto l(x) \cdot v\left[\gamma_{0}\right]$ by some $\mathbb{Z}\left[q, q^{-1}\right]$-linear form $l: H_{n}\left(F_{b}, \partial_{\infty} F_{b}\right) \rightarrow \mathbb{Z}\left[q, q^{-1}\right]$. Then $v^{-}\left[\gamma_{0}\right] \in$ $H_{n}\left(F_{b}, \partial_{0} F_{b}\right)$ is uniquely determined by the non-degeneracy of $(,)_{0}$ (cf. Proposition 8.1). If we replace the generator $v\left[\gamma_{0}\right]$ with $a \cdot v\left[\gamma_{0}\right]$ by some unit $a \in U\left(\mathbb{Z}\left[q, q^{-1}\right]\right)$, then the linear form $l$ should be replaced with $a^{-1} \cdot l=* a \cdot l$, and hence $v^{\sim}\left[\gamma_{0}\right]$ should be replaced with $a \cdot v^{\sim}\left[\gamma_{0}\right]$ by the hermitian property (8.1) of $(,)_{0}$. The uniqueness of the pair $\left(v\left[\gamma_{\infty}\right], v^{\vee}\left[\gamma_{\infty}\right]\right)$ modulo $U\left(\left[\mathbb{Z} q, q^{-1}\right]\right)$ is also derived from (9.2.3) and the primitiveness of $v^{\sim}\left[\gamma_{\infty}\right]$ in the same way.

Remark 9.2.3. Suppose that Theorem 9.2.1 holds for one simple loop $\gamma_{0}$ around $\mathscr{D}_{0}$ with the base point $b$. Then it holds for an arbitrary simple loop $\gamma_{0}^{\prime}$ around $\mathscr{D}_{0}$ with the base point $b^{\prime}$ arbitrarily chosen. Indeed, by Proposition 9.1.1, there exists a path $\alpha: I \rightarrow \mathcal{U}$ from $b^{\prime}$ to $b$ such that

$$
\left[\alpha^{-1} \gamma_{0} \alpha\right]=\left[\gamma_{0}^{\prime}\right] \text { in } \pi_{1}\left(\mathcal{U}, b^{\prime}\right)
$$

Let

$$
\begin{aligned}
& {[\alpha]_{*}: H_{n}\left(F_{b^{\prime}}, \partial_{\infty} F_{b^{\prime}}\right) \stackrel{\sim}{\rightarrow} H_{n}\left(F_{b}, \partial_{\infty} F_{b}\right), \quad \text { and }} \\
& {[\alpha]_{*}: H_{n}\left(F_{b^{\prime}}, \partial_{0} F_{b^{\prime}}\right) \stackrel{\sim}{\rightarrow} H_{n}\left(F_{b}, \partial_{0} F_{b}\right)}
\end{aligned}
$$

be the isomorphisms induced by the path $\alpha$. Then

$$
\left[\gamma_{0}^{\prime}\right]_{*}=[\alpha]_{*}^{-1} \circ\left[\gamma_{0}\right]_{*} \circ[\alpha]_{*} \text { on } H_{n}\left(F_{b^{\prime}}, \partial_{\infty} F_{b^{\prime}}\right) .
$$

By Remark 8.1, we have

$$
\left([\alpha]_{*}(x), v^{\sim}\left[\gamma_{0}\right]\right)_{0}=\left(x,[\alpha]^{-1}\left(v^{\sim}\left[\gamma_{0}\right]\right)\right)_{0}
$$


for all $x \in H_{n}\left(F_{b^{\prime}}, \partial_{\infty} F_{b^{\prime}}\right)$. Hence the formula (9.2.1) holds for $\left[\gamma_{0}^{\prime}\right]_{*}$ if we set

$$
v^{\sim}\left[\gamma_{0}^{\prime}\right]=[\alpha]_{*}^{-1}\left(v^{v}\left[\gamma_{0}\right]\right) \text {, and } v\left[\gamma_{0}^{\prime}\right]=[\alpha]^{-1}\left(v\left[\gamma_{0}\right]\right)
$$

It is obvious that if $v^{\sim}\left[\gamma_{0}\right]$ is primitive, then so is $[\alpha]_{*}^{-1}\left(v^{\sim}\left[\gamma_{0}\right]\right)$. The relation (9.2.2) also remains true for the pair $\left(v\left[\gamma_{0}^{\prime}\right], v^{\sim}\left[\gamma_{0}^{\prime}\right]\right)$ defined by (9.2.4).

Same argument is valid for Theorem 9.2.2.

It is therefore enough to prove each of Theorems 9.2 .1 and 9.2 .2 only for one suitably chosen simple loop.

Remark 9.2.4. Note that the complement $\mathscr{U} \backslash \mathcal{U}_{N}$ is of complex codimension 1 in $\mathcal{U}$. Note also that the complement $\mathcal{U} \backslash \mathcal{U}_{N}$ is of real codimension $\geq 1$ in $\mathcal{U}$. Combining these with Remark 9.2.3, we may assume that the base point $b$ is contained in $\mathscr{U}_{N}^{\tilde{N}}$, and the simple loops $\gamma_{0}$ and $\gamma_{\infty}$ are contained in $\boldsymbol{U}_{N}$.

\section{\$9.3. Deformation of Thimbles}

Taking Remark 9.2.4 into account, we will descride a method for calculating the action of $[\gamma] \in \pi_{1}\left(U_{N}, b\right)$ with $b \in \mathcal{U}_{N}^{\sim}$ on $H_{n}\left(F_{b}, \partial_{0} F_{b}\right)$ and $H_{n}\left(F_{b}, \partial_{\infty} F_{b}\right)$.

Let $\gamma: I \rightarrow U_{N}$ be a loop with the base point $b$. By the definition of $U_{N}$, the fundamental group $\pi_{1}\left(U_{N}, b\right)$ acts on the sets $\operatorname{Cr}(b) \subset \mathbb{C}^{\times}$and $\mathscr{C} r(b) \subset \mathbb{C}$. Let $[\gamma]_{*}: \mathrm{Cr}(b) \rightarrow \mathrm{Cr}(b)$ and $[\gamma]_{*}: \mathscr{C} r(b) \rightarrow \mathscr{C} r(b)$ denote the actions of $[\gamma] \in$ $\pi_{1}\left(U_{N}, b\right)$. For $u \in U_{N}$, we put

$$
\mathscr{S}_{u}:=\mathscr{C} r(u) \cup Z_{u}^{0} \cup Z_{u}^{\infty} \subset \mathbb{C}
$$

where $Z_{u}^{0}=\left\{a_{u}^{0}\langle\nu\rangle ; \nu \in \mathbb{Z}\right\}$ and $Z_{u}^{\infty}=\left\{a_{u}^{\infty}\langle\nu\rangle ; \nu \in \mathbb{Z}\right\}$, and call it the set of distinguished points. Then the points of $\mathscr{S}_{r(t)}$ move on $\mathbb{C}$ continuously when $t$ varies, and any two distinct points do not collide during this movement because of the definitions of $U_{N}$ and $Z_{u}^{0}, Z_{u}^{\infty}$. Moreover, $[\gamma]_{*}$ acts on both of $Z_{b}^{0}$ and $Z_{b}^{\infty}$ trivially. Hence we can denote this movement by the continuous map

$$
M_{r}: I \times \varkappa_{b} \rightarrow \mathbb{C}
$$

which satisfies the following:

(1) $M_{r}(0, s)=s$ for all $s \in \mathscr{S}_{b}$,

(2) $M_{r}(1, \tilde{p})=[r]_{*}(\tilde{p})$ for $p \in \mathscr{C}_{r}(b)$,

(3) $M_{r}\left(t, a_{b}^{0}\langle\nu\rangle\right)=a_{\gamma(t)}^{0}\langle\nu\rangle, M_{r}\left(t, a_{b}^{\infty}\langle\nu\rangle\right)=a_{\gamma(t)}^{\infty}\langle\nu\rangle$ for all $\nu \in \mathbb{Z}$ and $t \in I$, and

(4) $\mathcal{M}_{r}(t, \cdot): \mathscr{\bigotimes}_{b} \rightarrow \mathbb{C}$ is injective for all $t \in I$. 
We consequently obtain, for each critical value $\tilde{p} \in \mathscr{C} r(b)$, the bijective maps of the sets of homotopy classes of paths

$$
\begin{aligned}
& {[\gamma]_{*}:\left[\mathscr{P}_{b}^{\sim}\left(a_{b}^{0}\langle\nu\rangle, \tilde{p}\right)\right] \stackrel{\sim}{\rightarrow}\left[\mathscr{P}_{b}^{\sim}\left(a_{b}^{0}\langle\nu\rangle,[\gamma]_{*}(\tilde{p})\right)\right], \quad \text { and }} \\
& {[\gamma]_{*}:\left[\mathscr{P}_{b}^{\sim}\left(a_{b}^{\infty}\langle\nu\rangle, \tilde{p}\right)\right] \stackrel{\sim}{\rightarrow}\left[\mathscr{P}_{b}^{\sim}\left(a_{b}^{\infty}\langle\nu\rangle,[\gamma]_{*}(\tilde{p})\right)\right]}
\end{aligned}
$$

induced by the movement $\mathcal{M}_{r}$.

Now suppose that we are given a path $\widetilde{\omega} \in \mathscr{P}_{b}^{\sim}\left(a_{b}^{0}\langle\nu\rangle, \tilde{p}\right)$. We choose a vanishing cycle $\sigma[\widetilde{\omega}] \in\left[S^{n-1}, X_{b}^{0}\langle\nu\rangle\right]$ for $[\widetilde{\omega}] \in\left[\mathscr{P}_{b}^{\sim}\left(a_{b}^{0}\langle\nu\rangle, \tilde{p}\right)\right]$, and consider the thimble

$$
\theta([\widetilde{\omega}], \sigma[\widetilde{\omega}]) \in\left[\left(C S^{n-1}, S^{n-1}\right),\left(F_{b}, X_{b}^{0}\langle\nu\rangle\right)\right]
$$

for $[\widetilde{\omega}]$ starting from $\sigma[\widetilde{\omega}]$. We have natural actions

$$
[\gamma]_{*}:\left[\left(C S^{n-1}, S^{n-1}\right),\left(F_{b}, X_{b}^{0}\langle\nu\rangle\right)\right] \stackrel{\sim}{\rightarrow}\left[\left(C S^{n-1} \cdot S^{n-1}\right),\left(F_{b}, X_{b}^{0}\langle\nu\rangle\right)\right]
$$

and

$$
[\gamma]_{*}:\left[S^{n-1}, X_{b}^{0}\right] \stackrel{\sim}{\rightarrow}\left[S^{n-1}, X_{b}^{0}\right],[\gamma]_{*}:\left[S^{n-1}, X_{b}^{0}\langle\nu\rangle\right] \stackrel{\sim}{\rightarrow}\left[S^{n-1}, X_{b}^{0}\langle\nu\rangle\right]
$$

By the definition, $[\gamma]_{*}(\sigma[\widetilde{\omega}]) \in\left[S^{n-1}, X_{b}^{0}\langle\nu\rangle\right]$ is one of the vanishing cycles for $[\gamma]_{*}([\widetilde{\omega}]) \in\left[\mathscr{P}_{b}^{\sim}\left(a_{b}^{0}\langle\nu\rangle,[\gamma]_{*}(\tilde{p})\right)\right]$, and we have a formula

$$
[\gamma]_{*}(\theta([\widetilde{\omega}], \sigma[\widetilde{\omega}]))=\theta\left([\gamma]_{*}([\widetilde{\omega}]),[\gamma]_{*}(\sigma[\widetilde{\omega}])\right)
$$

In particular, we have

$$
[\gamma]_{*}(\theta([\widetilde{\omega}]))= \pm \theta\left([\gamma]_{*}([\widetilde{\omega}])\right)
$$

because the thimble is determined uniquely up to sign by the homotopy class of the underlying path. The vanishing cycle $[\gamma]_{*}(\sigma[\widetilde{\omega}])$ in $(9.3 .4)$ is calculated by looking at the action $[\gamma] *$ on the set $\left[S^{n-1}, X_{b}^{0}\right]$. Indeed, the path $\widetilde{\omega}$ is the lift $\omega\langle\nu\rangle$ of the path $\omega:=e \circ \widetilde{\omega} \in \mathscr{P}_{b}\left(a_{b}^{0}, p\right)$, where $p=e(\tilde{p})$, and the vanishing cycle $\sigma[\widetilde{\omega}]$ is the lift $\sigma[\omega]\langle\nu\rangle$ of $\sigma[\omega] \in\left[S^{n-1}, X_{b}^{0}\right]$. The two actions in (9.3.3) are compatible via the isomorphism $\left[S^{n-1}, X_{b}^{0}\langle\nu\rangle\right] \cong\left[S^{n-1}, X_{b}^{0}\right]$ given by (6.1). Hence we have 


$$
[\gamma]_{*}(\sigma[\widetilde{\varpi}])=\left([\gamma]_{*}(\sigma[\omega])\right)\langle\nu\rangle .
$$

By Proposition 7.3, $H_{n}\left(F_{b}, \partial_{0} F_{b}\right)$ is generated by the homology classes of thimbles for the homotopy classes of paths from $a_{b}^{0}\langle 0\rangle$ to values in $\mathscr{C}_{r}(b)$. Hence the formulae (9.3.4) and (9.3.6) enable us to calculate the action of $[\gamma] \in$ $\pi_{1}\left(\mathscr{U}_{N}, b\right)$ on $H_{n}\left(F_{b}, \partial_{0} F_{b}\right)$ by looking at the map $[\gamma]_{*}:\left[\mathscr{P}_{b}^{\sim}\left(a_{b}^{0}\langle 0\rangle, \tilde{p}\right)\right] \stackrel{\sim}{\rightarrow}$ $\left[\mathscr{P}_{b}^{\sim}\left(a_{b}^{0}\langle 0\rangle,[\gamma]_{*}(\tilde{p})\right)\right]$ for $\tilde{p} \in \mathscr{C}_{r}(b)$ and the action $[\gamma]_{*}:\left[S^{n-1}, X_{b}^{0}\right] \stackrel{\sim}{\rightarrow}$ $\left[S^{n-1}, X_{b}^{0}\right]$.

Same argument holds when 0 is replaced with $\infty$.

In order to investigate the maps (9.3.1), we introduce the notion of homotopy equivalence of movements of points on $\mathbf{C}$.

Definition 9.3.1. Let $\mathcal{M}_{0}: I \times \& \rightarrow \mathrm{C}$ and $\mathcal{M}_{1}: I \times \& \rightarrow \mathrm{C}$ be two movements of a set of points $\&$ on $\mathrm{C}$ such that

(i) $\mathcal{M}_{0}(0, s)=\mathcal{M}_{1}(0, s)$ for all $s \in \&$,

(ii) $\mathcal{M}_{0}(1, s)=\mathcal{M}_{1}(1, s)$ for all $s \in \mathscr{S}$, and

(iii) for all $t$, both of the maps from $\&$ to $\mathbb{C}$ given by $s \mapsto \mathcal{M}_{0}(t, s)$ and by $s \mapsto$ $\mathcal{M}_{1}(t, s)$ are injective.

These two movements are said to be homotopically equivalent if there exists a continuous map $\mathfrak{M}: I \times I \times \& \rightarrow \mathbb{C}$ such that the movements $\mathcal{M}(\tau):=\mathfrak{M}(\tau, \cdot, \cdot)$ : $I \times \& \rightarrow \mathrm{C}$ satisfy the following:

(1) $M(0)=M_{0}, M(1)=M_{1}$,

(2) $\mathcal{M}(\tau)(0, s)=\mathcal{M}_{0}(0, s)=\mathcal{M}_{1}(0, s)$ for all $\tau \in I$ and $s \in \mathscr{S}$,

(3) $\mathcal{M}(\tau)(1, s)=\mathcal{M}_{0}(1, s)=\mathcal{M}_{1}(1, s)$ for all $\tau \in I$ and $s \in \mathscr{S}$, and

(4) $\mathcal{M}(\tau)(t, \cdot): \& \rightarrow \mathbb{C}$ is injective for all $(\tau, t) \in I \times I$.

It is obvious that the maps (9.3.1) depend only on the homotopy class of the movement $\mathcal{M}_{r}$. Therefore, we will find a simpler movement in the homotopy equivalence class containing $\boldsymbol{M}_{r}$.

Reduction 1. Note that, for all $\tilde{p} \in \mathscr{C}_{r}(b)$ and for all $t \in I$, the point $\mathcal{M}_{r}(t, \tilde{p})$ remains on the right-hand side of the vertical line $R_{r(t)}^{0}$, which contains the points $a_{\gamma(t)}^{0}\langle\nu\rangle=\mathcal{M}_{r}\left(t, a_{b}^{0}\langle\nu\rangle\right)$, and on the left-hand side of the vertical line

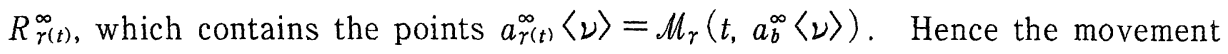
$\mathcal{M}_{r}$ is always homotopically equivalent to a movement $\mathcal{M}_{r}^{\prime}$ such that

$$
\mathcal{M}_{r}^{\prime}\left(t, a_{b}^{0}\langle\nu\rangle\right)=a_{b}^{0}\langle\nu\rangle \text { and } \mathcal{M}_{r}^{\prime}\left(t, a_{b}^{\infty}\langle\nu\rangle\right)=a_{b}^{\infty}\langle\nu\rangle \text { for all } t \in I .
$$

This reduction is also obtained by using the following:

Remark 9.3.1. We choose a small positive real number $r$, and use $\min \{\widetilde{\varepsilon} / 2, r\}$ 
as the function $\varepsilon$ from now on to the end of this paper. All the loops and the paths on $\mathcal{U}$ which will appear in the argument of this paper will be defined without using this chosen number $r$, and their number is finte. Each loop or path is compact, and hence their union is also compact. Therefore, taking $r$ sufficiently small, we can assume that $\varepsilon(\alpha(t))$ is constantly equal with $r$ for every loop or path $\alpha: I \rightarrow \mathcal{U}$ which will appear from now on. In particular, the points $a_{\alpha(t)}^{0}, a_{\alpha(t)}^{\infty} \in$ $\mathbb{C}^{\times}$, and $a_{\alpha(t)}^{0}\langle\nu\rangle, a_{\alpha(t)}^{\infty}\langle\nu\rangle \in \mathbb{C}$ do not move.

Reduction 2. Suppose that there exists a subset $J$ of $\{1, \ldots, N\}$ with satisfies the following: (i) $[\gamma]_{*}\left(p_{j}\right)=p_{j}$ for all $j \in J$, and (ii) for each $j \in J$, there exists a continuous map $g_{j}: \Delta \rightarrow \mathbb{C}^{\times}$from a closed unit disk $\Delta$ such that $g,(\partial \Delta)$ coincides with the loop drawn by the movement of the critical value $p_{3}$ on $\mathbb{C}^{\times}$, and that $g_{j}(\Delta)$ is disjoint from the trace of the movement of any other critical value $p_{l}(i \neq j)$ on $\mathbb{C}^{\times}$. Then we have $[\gamma]_{*}\left(p_{j}\langle\nu\rangle\right)=p_{j}\langle\nu\rangle$ for all $j \in J$ and all $\nu \in \mathbb{Z}$. Moreover, the movement $M_{r}$ is homotopically equivalent to $\mathcal{M}_{r}^{\prime}$ which has, in addition to (9.3.7), the following property : if $j \in J$, then $\mathcal{M}_{r}^{\prime}(t$, $\left.p_{j}\langle\nu\rangle\right)=p_{j}\langle\nu\rangle$ for all $t \in I$ and all $\nu \in \mathbb{Z}$, while if $i \notin J$, then $\mathcal{M}_{r}^{\prime}\left(t, p_{i}\langle\nu\rangle\right)=$ $\mathcal{M}_{r}\left(t, p_{i}\langle\nu\rangle\right)$ for all $\nu \in \mathbb{Z}$; that is, the movements of $p_{j}\langle\nu\rangle$ with $j \in J$ can be deformed to the non-movement without affecting the movements of the other critical values $p_{i}\langle\nu\rangle(i \notin J)$.

Remark 9.3.2. Note that $[\gamma] \in \pi_{1}\left(U_{N}, b\right)$ induces a bijective map from $\left[\mathscr{P}_{b}\left(a_{b}^{0}, p\right)\right]$ to $\left[\mathscr{P}_{b}\left(a_{b}^{0},[\gamma]_{*}(p)\right)\right]$. It is obvious that this action is compatible with the action (9.3.1) via the lifting; that is, we have

$$
[\gamma]_{*}([\omega]\langle\nu\rangle)=\left([\gamma]_{*}([\omega])\right)\langle\nu\rangle \text { for all }[\omega] \in\left[\mathscr{P}_{b}\left(a_{b}^{0}, p\right)\right]
$$

We also have a natural action of $[\gamma] \in \pi_{1}\left(U_{N}, b\right)$ on $\left[\left(C S^{n-1}, S^{n-1}\right),\left(E_{b}, X_{b}^{0}\right)\right]$, which is compatible with (9.3.2) via the lifting; that is,

$$
\begin{aligned}
{[\gamma]_{*}(\theta([\omega\langle\nu\rangle],} & \sigma[\omega\langle\nu\rangle]))=\left([\gamma]_{*}(\theta([\omega], \sigma[\omega]))\right)\langle\nu\rangle \\
& \text { for all } \theta([\omega], \sigma[\omega]) \in\left[\left(C S^{n-1}, S^{n-1}\right),\left(E_{b}, X_{b}^{0}\right)\right] .
\end{aligned}
$$

Same argument holds when 0 is replaced with $\infty$.

\section{\$9.4. Proof of Theorem 9.2 .1}

We fix a point $u$ of $\boldsymbol{U}_{N}$.

Recall that $\mathscr{L}_{u} \subset \Gamma^{\times}$is the affine line $\left\{f_{u}-t \cdot h^{d} ; t \in \mathbb{C}\right\}$ with the parameterization $\iota_{u}: \mathbb{C} \stackrel{\sim}{\rightarrow} \mathscr{L}_{u}$ given by $t \mapsto f_{u}-t \cdot h^{d}$. Let $w$ be an arbitrary point on 
$\mathscr{L}_{u}$. By definition, the affine line $\mathscr{L}_{w}$ is equal with $\mathscr{L}_{u}$, and we write this affine line simply by $\mathscr{L}$. By Lemma 2.3 , we have

$$
\mathscr{L} \backslash \mathscr{D}_{0}=\mathscr{L} \cap \mathcal{U}=\mathscr{L} \cap \mathcal{U}_{N}
$$

Let $c_{1}, \ldots, c_{N}$ be the intersection points of $\mathscr{L}$ and $\mathscr{D}_{0}$. Then, by Corollary 2.1 , the critical values of $\widehat{\phi}_{w}$ are accordingly numbered;

$$
\operatorname{Cr}(w)=\left\{p_{1}(w), \ldots . p_{N}(w)\right\}, \text { where } p_{\imath}(w)=\iota_{w}^{-1}\left(c_{\imath}\right)
$$

The point $w$ is on $\mathscr{L} \cap \mathscr{D}_{0}=\mathscr{L} \backslash \mathcal{U}_{N}$ if and only if one of $p_{1}(w), \ldots, p_{N}(w)$ is zero.

Lemma 9.4.1. We have $p_{i}(w)=p_{i}(u)+s_{w}$ for $i=1, \ldots, N$, where $s_{w}:=\iota_{w}^{-1}(u)$ $=-\iota_{u}^{-1}(w)$. In particular, $p_{\imath}(u)-p_{j}(u)=p_{\imath}(w)-p_{j}(w)$ holds for all $w \in \mathscr{L}$.

Proof. The two parameterizations $\iota_{u}: \mathbf{C} \rightarrow \mathscr{L}$ and $\iota_{w}: \mathbf{C} \rightarrow \mathscr{L}$ differ only by an additive constant, and an easy calculation shows that $\iota_{w}^{-1} \circ \iota_{u}(s)=s+s_{w}$.

This lemma shows that the set $\operatorname{Cr}(w) \subset \mathbb{C}$ moves by parallel translation when $w$ moves on $\mathscr{L}$.

Let $\rho$ be a complex number with $\rho \notin \mathbb{R}$ and $|\rho|$ small enough. We choose the point

$$
b:=\iota_{u}\left(p_{1}(u)-\rho\right) \in \mathscr{L} \backslash \mathscr{D}_{0} \subset \mathcal{U}_{N}
$$

as the base point, so that $p_{1}(b)=\rho$. Since $|\rho|$ is sufficiently small and $\operatorname{Im} \rho \neq 0$, we may assume that none of $c_{1}, \ldots, c_{N}$ is on the real semi-line $\iota_{u}\left(p_{1}(u)-\rho+\mathbb{R}_{\leq 0}\right)$; that is

$$
b \in \mathcal{U}_{N}^{\tilde{N}}
$$

In particular, we have $K_{b} \subset \mathbb{C}^{\times}$and the isomorphisms $\Psi_{b}^{0}, \Psi_{b}^{\infty}$. By Lemma 9.4.1, we have

$$
p_{i}(b)=p_{\imath}(u)-p_{1}(u)+\rho
$$

Since $|\rho|$ is small enough, this implies that

$$
\left|p_{\imath}(b)\right|>3 \cdot|\rho| \text { if } i \neq 1 \text {, and }\left|p_{\imath}(b)-p_{j}(b)\right|>3 \cdot|\rho| \text { if } i \neq j \text {. }
$$


Now we consider the closed disk $\Delta$ on $\mathscr{L}$ with the center $\iota_{b}\left(p_{1}(b)\right)=\iota_{b}(\rho)$ $=c_{1}$ and of radius $|\rho|$. The base point $b=\iota_{b}(0)$ is located on the boundary $\partial \Delta$. Since $|\rho|$ is small enough, the intersection $\Delta \cap \mathscr{D}_{0}$ consists of only one point $c_{1}$. Moreover, since $u \in \mathscr{U}_{N}, \mathscr{L}$ intersects $\mathscr{D}_{0}$ transversely by Proposition 2.4. The loop $\gamma: I \rightarrow \partial \Delta \subset \mathscr{L}$ given by

$$
\gamma(t):=\iota_{b}\left(p_{1}(b)-\rho e^{2 \pi \sqrt{-1} t}\right)=\iota_{b}\left(\rho\left(1-e^{2 \pi \sqrt{-1} t}\right)\right)
$$

is therefore a simple loop around $\mathscr{D}_{0}$ with the base point $b$. By (9.4.1), we have

$$
\gamma(I) \subset \mathcal{U}_{N}
$$

Note that the number $r$ which we have chosen in Remark 9.3.1 is small enough even compared with $|\rho|$, and hence we have $a_{\gamma(t)}^{0}=r$ and $a_{\gamma(t)}^{\infty}=1 / r$ for all $t \in I$.

Let $D_{1} \subset \mathbb{C}$ be the closed disk with the center 0 and of radius $|\rho|$. The critical value $p_{1}(b)=\rho$ is located on the boundary of this disk. We see from (9.4.5) that $D_{1} \cap \mathrm{Cr}(b)$ consists of only one point $p_{1}(b)$. Note also that $D_{1} \cap$ $K_{b}$ is simply-connected. Therefore, there exists a unique homotopy class $\left[\xi_{1}^{0}\right]$ $\in\left[\mathscr{P}_{b}\left(a_{b}^{0}, p_{1}(b)\right)\right]$ of paths which is represented by a path $\xi_{1}^{0}$ such that

$$
\xi_{1}^{0}(I) \subset D_{1} \cap K_{b}
$$

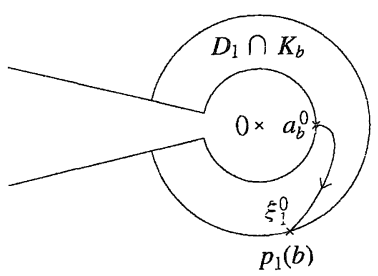

Figure 7

Now, by Remarks 9.2.2 and 9.2.3, Theorem 9.2.1 follows from the following:

Proposition 9.4.1. Let

$$
v^{\circ}:=\bar{\theta}\left(\left[\xi_{1}^{0}\right]\right)\langle 0\rangle \in H_{n}\left(F_{b}, \partial_{0} F_{b}\right)
$$

be the homology class of the lifted thimble $\theta\left(\left[\xi_{1}^{0}\right]\right)\langle 0\rangle$, where $\left[\xi_{1}^{0}\right] \in\left[\mathscr{P}_{b}\left(a_{b}^{0}\right.\right.$, $\left.\left.p_{1}(b)\right)\right]$ is the unique homotopy class of paths characterized by (9.4.8). We define the element $v$ of $H_{n}\left(F_{b}\right)$ by 


$$
v:=(-1)^{n(n-1) / 2}(q-1) v^{\circ}
$$

using (6.16). Then, $v^{-}$is primitive, and the local monodromy action $[\gamma] *$ on $H_{n}\left(F_{b}, \partial_{\infty} F_{b}\right)$ along the simple loop $\gamma$ around $\mathscr{D}_{0}$ given by $(9.4 .6)$ is written as follows:

$$
x \mapsto x+\left(x, v^{\sim}\right)_{0} \cdot v
$$

Proof. By Lemma 9.4.1 and (9.4.6), we have

$$
p_{i}(\gamma(t))=p_{i}(b)-\rho\left(1-e^{2 \pi \sqrt{-1} t}\right)
$$

This means that, when $t$ moves from 0 to 1 , each $p_{i}(\gamma(t))$ draws a circle of the radius $|\rho|$ with the center $p_{1}(b)-\rho$ in the counter-clockwise direction. Let $C_{2}$ denote this circle, and $D_{i}$ the disk circumscribed by $C_{i}$. Note that this $D_{1}$ coincides with that $D_{1}$ which we have defined just before the statement of Proposition 9.4.1. By $(9.4 .7),[\gamma] *$ acts on the set $\mathscr{C} r(b)$. By (9.4.3), each value in $\mathscr{C} r(b)$ is written in the form $p_{\imath}(b)\langle\nu\rangle$, where $i=1, \ldots, N$ and $\nu \in \mathbb{Z}$. We see from (9.4.5) that

$$
D_{\imath} \nRightarrow 0 \quad \text { if } \quad i \neq 1 \text {. }
$$

On the other hand, we see that $D_{1} \ni 0$, and the circle $C_{1}$ traverses $\mathbb{R}_{\leq 0}$ in the positive direction. Hence we have

$$
[\gamma]_{*}\left(p_{\imath}(b)\langle\nu\rangle\right)= \begin{cases}p_{\imath}(b)\langle\nu\rangle & \text { if } i \neq 1, \text { and } \\ p_{1}(b)\langle\nu+1\rangle & \text { if } i=1 .\end{cases}
$$

By (9.4.3), we have a $K$-regular system $\left\{\xi_{1}^{\infty}, \ldots, \xi_{N}^{\infty}\right\}$ of paths from $a_{b}^{\infty}$. We see from (9.4.5) that

$$
D_{i} \cap D_{j}=\varnothing \quad \text { if } \quad i \neq j .
$$

It is therefore possible to take the $K$-regular system in such a way that

$$
\xi_{i}^{\infty}(I) \cap D_{1}= \begin{cases}\varnothing & \text { if } i \neq 1, \text { and } \\ \left\{p_{1}(b)\right\} & \text { if } i=1 .\end{cases}
$$

Now we choose a vanishing cycle $\sigma_{i}^{\infty}:=\sigma\left[\xi_{i}^{\infty}\right] \in\left[S^{n-1}, X_{b}^{\infty}\right]$ for each $\left[\xi_{i}^{\infty}\right]$ from among the two possibilities, and consider the lift of the associated thimble 


$$
\theta_{i}^{\infty}\langle 0\rangle:=\theta\left(\left[\xi_{i}^{\infty}\right], \sigma_{i}^{\infty}\right)\langle 0\rangle \in\left[\left(C S^{n-1}, S^{n-1}\right),\left(F_{b}, X_{b}^{\infty}\langle 0\rangle\right)\right],
$$

which is the thimble for $\left[\xi_{i}^{\infty}\langle 0\rangle\right]$ starting from the lifted vanishing cycle $\sigma_{\imath}^{\infty}\langle 0\rangle$ in $X_{b}^{\infty}\langle 0\rangle$. Since the homology classes $\bar{\theta}_{1}^{\infty}\langle 0\rangle, \ldots, \bar{\theta}_{N}^{\infty}\langle 0\rangle$ of these thimbles form a set of basis of $H_{n}\left(F_{b}, \partial_{\infty} F_{b}\right)$ by Proposition 7.3, it is enough to prove (9.4.10) when $x$ runs through the set of these classes.

The intersection number $\left(\bar{\theta}_{i}^{\infty}\langle 0\rangle, v^{\sim}\right)_{0} \in \mathbb{Z}\left[q, q^{-1}\right]$ is calculated as follows :

Claim $\mathbb{1}$.

$$
\left(\bar{\theta}_{i}^{\infty}\langle 0\rangle, v^{-}\right)_{0}=\left(\bar{\theta}_{i}^{\infty}\langle 0\rangle, \bar{\theta}\left(\left[\xi_{1}^{0}\right]\right)\langle 0\rangle\right)_{0}= \begin{cases}0 & \text { if } i \neq 1 \text {, and } \\ \pm 1 & \text { if } i=1 .\end{cases}
$$

Proof. Because of (9.4.8) and (9.4.15), we can derive Claim 1 from Lemma 8.2.

This claim, in particular, shows that $v^{\sim}$ is primitive.

We choose the sign of the vanishing cycle $\sigma_{1}^{\infty}$ for $\left[\xi_{1}^{\infty}\right]$ in such a way that

$$
\left(\bar{\theta}_{1}^{\infty}\langle 0\rangle, v^{\sim}\right)_{0}=1
$$

Claim 2. The monodromy action $[\gamma] *$ on $\left[S^{n-1}, X_{b}^{\infty}\right]$ is trivial.

Proof. We see that

$$
\begin{aligned}
X_{r(t)}^{\infty} & =\phi_{r(t)}^{-1}\left(a_{r(t)}^{\infty}\right) & & \\
& =\phi_{r(t)}^{-1}(1 / r) & & \text { by Remark } 9.3 .1 \\
& =\phi_{b}^{-1} \circ \iota_{b}^{-1} \circ \iota_{r(t)}(1 / r) & & \text { by }(2.3) \\
& =\phi_{b}^{-1}\left(1 / r+\rho-\rho e^{2 \pi \sqrt{-1} t}\right) & & \text { by }(9.4 .6) ;
\end{aligned}
$$

that is, the family $\left\{X_{\gamma(t)}^{\infty} ; t \in I\right\}$ over $\partial \Delta=\gamma(I)$ is isomorphic to the restriction of $\phi_{b}: E_{b} \rightarrow \mathbb{C}^{\times}$to the circle $C_{\infty} \subset \mathbb{C}^{\times}$of radius $|\rho|$ with the center $1 / r+\rho$. Since $r$ can be taken arbitrarily small, this circle can be far away from 0 as much as we want. Thus we can conclude that the disk $D_{\infty}$ circumscribed by $C_{\infty}$ does not contain any critical values of $\widehat{\phi}_{b}$. Hence Claim 2 follows from Proposition 2.1. 
Claim 3. $[\gamma]_{*}\left(\left[\xi_{i}^{\infty}\langle 0\rangle\right]\right)=\left[\xi_{i}^{\infty}\langle 0\rangle\right]$ for $i=2, \ldots, N$.

Proof. By (9.4.12), (9.4.14) and Reductions 1 and 2 in $§ 9.3$, the movement $\mathcal{M}_{r}$ of the distinguished points $\varkappa_{b}$ is homotopically equivalent to a mavement $\mathcal{M}_{r}^{\prime}$ which remains $a_{b}^{0}\langle\nu\rangle, a_{b}^{\infty}\langle\nu\rangle$ fixed for all $\nu \in \mathbb{Z}$, and $p_{\imath}\langle\nu\rangle$ also fixed for $i=$ $2, \ldots, N$ and for all $\nu \in \mathbb{Z}$, while it moves $p_{1}(b)\langle\nu\rangle$ to $p_{1}(b)\langle\nu+1\rangle$ along the vertical line $\log |\rho|+\sqrt{-1} \mathbf{R}=e^{-1}\left(\partial D_{1}\right)$. If $i \neq 1$, then the path $\xi_{i}^{\infty}\langle 0\rangle$ is disjoint from this vertical line because of (9.4.15), and hence it is not affected by the movement of $p_{1}(b)\langle\nu\rangle$. Therefore we obtain the claim.

Applying Claims 2 and 3 to the formulae (9.3.4) and (9.3.6), we obtain

$$
[\gamma]_{*}\left(\theta_{l}^{\infty}\langle 0\rangle\right)=\theta_{i}^{\infty}\langle 0\rangle \text { for } i=2, \ldots, N
$$

We put

$$
v^{\prime}:=[\gamma]_{\times}\left(\bar{\theta}_{1}^{\infty}\langle 0\rangle\right)-\bar{\theta}_{1}^{\infty}\langle 0\rangle \in H_{n}\left(F_{b}, \partial_{\infty} F_{b}\right)
$$

By Claim 1, the choice of sign (9.4.16), and (9.4.17), we see that

$$
[\gamma]_{*}(x)=x+\left(x, v^{\sim}\right)_{0} \cdot v^{\prime} \text { for all } x \in H_{n}\left(F_{b}, \partial_{\infty} F_{b}\right)
$$

Now we shall prove that $v$ is equal with $(-1)^{n(n-1) / 2}(q-1) v^{v} \in H_{n}\left(F_{b}\right)$, and prove $(9.4 .9)$. First remark that the formulae (9.3.4), (9.3.6) and Claim 2 imply that

$$
[\gamma]_{*}\left(\theta_{1}^{\infty}\langle\nu\rangle\right)=\theta\left([\gamma]_{*}\left(\left[\xi_{1}^{\infty}\langle\nu\rangle\right]\right),[\gamma]_{*}\left(\sigma_{1}^{\infty}\langle\nu\rangle\right)\right)=\theta\left([\gamma]_{*}\left(\left[\xi_{1}^{\infty}\langle\nu\rangle\right]\right), \sigma_{1}^{\infty}\langle\nu\rangle\right) .
$$

Because of $(9.4 .13)$, the homotopy class $[\gamma]_{*}\left(\left[\xi_{1}^{\infty}\langle\nu\rangle\right]\right)$ of paths is an element of

$$
\left[\mathscr{P}_{b}^{\sim}\left(a_{b}^{\infty}\langle\nu\rangle, p_{1}(b)\langle\nu+1\rangle\right)\right]
$$

Now we shall describe paths which represent this homotopy class.

By the description of the movement $\mathcal{M}_{r}^{\prime}$ in the proof of Claim 3, the homotopy class $[\gamma]_{*}\left(\left[\xi_{1}^{\infty}\langle\nu\rangle\right]\right)$ is represented by a path $\xi^{\prime}\langle\nu\rangle$ defined as follows. Note that by $(9.4 .15)$, the path $\xi_{1}^{\infty}\langle\nu\rangle$ is on the right-hand side of the vertical line 


$$
\Lambda_{0}:=\log |\rho|+\sqrt{-1} \mathbb{R}
$$

along which the points $p_{1}(\gamma(t))\langle\nu\rangle=\log |\rho|+\sqrt{-1}(\nu+\arg \rho+t)$ moves. Then $\xi^{\prime}\langle\nu\rangle$ starts from $a_{b}^{\infty}\langle\nu\rangle$, goes to a point $p_{1}^{\prime}\langle\nu\rangle:=p_{1}(b)\langle\nu\rangle+\kappa$ along $\xi_{1}^{\infty}\langle\nu\rangle$, where $\kappa$ is a sufficiently small complex number with Re $\kappa>0$, draws an arc in the counter-clockwise direction to the point $p_{1}(b)\langle\nu\rangle+\sqrt{-1}|\kappa|$ on the line $\Lambda_{0}$ along the circle of radius $|\kappa|$ with the center $p_{1}(b)\langle\nu\rangle$, and goes to $p_{1}(b)\langle\nu+1\rangle$ along $\Lambda_{0}$.

Let $\xi_{1}^{\infty 0}\langle\nu\rangle$ be the path on $\mathbb{C} \backslash \mathscr{C} r(b)$ from $a_{b}^{\infty}\langle\nu\rangle$ to $a_{b}^{0}\langle\nu\rangle$ defined as follows. Note that, by (9.4.8), the path $\xi_{1}^{0}\langle\nu\rangle$ is on the left-hand side of the vertical line $\Lambda_{0}$. Then the path $\xi_{1}^{\infty 0}\langle\nu\rangle$ starts from $a_{b}^{\infty}\langle\nu\rangle$, goes to $p_{1}^{\prime}\langle\nu\rangle$ along $\xi_{1}^{\infty}\langle\nu\rangle$, draws an arc on the circle of radius $|\kappa|$ with the center $p_{1}(b)\langle\nu\rangle$ in the counter-clockwise direction to the point $p_{1}(b)\langle\nu\rangle-\kappa^{\prime}$ on $\xi_{1}^{0}\langle\nu\rangle(I)$, where $\kappa^{\prime}$ is a certain complex number with $\left|\kappa^{\prime}\right|=|\kappa|$ and $\operatorname{Re} \kappa^{\prime}>0$, and goes to $a_{b}^{0}\langle\nu\rangle$ along $\xi_{1}^{0}\langle\nu\rangle^{-1}$. It is easy to see that

$$
\begin{aligned}
{[\gamma]_{*}\left(\left[\xi_{1}^{\infty}\langle\nu\rangle\right]\right)=\left[\xi^{\prime}\langle\nu\rangle\right]=\left[\xi_{1}^{0}\langle\nu+1\rangle \cdot\right.} & \left.\delta_{b}^{0}\langle\nu\rangle \cdot \xi_{1}^{\infty}\langle\nu\rangle\right] \text { in } \\
& {\left[\mathscr{P}_{b}^{\sim}\left(a_{b}^{\infty}\langle\nu\rangle, p_{1}(b)\langle\nu+1\rangle\right)\right] . }
\end{aligned}
$$

(See $\S 6$ for the definition of the path $\delta_{b}^{0}$.) We put

$$
\widetilde{\xi}\langle 0\rangle:=\xi_{1}^{0}\langle 1\rangle \cdot \delta_{b}^{0}\langle 0\rangle \cdot \xi_{1}^{\infty}\langle 0\rangle
$$

Then, from (9.4.18), we have

$$
[\gamma]_{*}\left(\theta_{1}^{\infty}\langle 0\rangle\right)=\theta\left([\widetilde{\xi}\langle 0\rangle], \sigma_{1}^{\infty}\langle 0\rangle\right) .
$$

We decompose the path $\xi_{1}^{\infty}\langle 0\rangle$ into two parts at $p_{1}^{\prime}\langle 0\rangle$; that is, we write $\xi_{1}^{\infty}\langle 0\rangle$ $=\eta_{2} \cdot \eta_{1}$, where $\eta_{1}$ is the path from $a_{b}^{\infty}\langle 0\rangle$ to $p_{1}^{\prime}\langle 0\rangle$ along $\xi_{1}^{\infty}\langle 0\rangle$, and $\eta_{2}$ is the remaining part. Then $\xi_{1}^{\infty}\langle 0\rangle$ also decomposes into $\eta_{3} \cdot \eta_{1}$. Let

$$
T: C S^{n-1} \rightarrow F_{b}, \quad \text { and } \quad T_{\gamma}: C S^{n-1} \rightarrow F_{b}
$$

be continuous maps representing $\theta_{1}^{\infty}\langle 0\rangle$ over $\xi_{1}^{\infty}\langle 0\rangle$ and $[\gamma]_{*}\left(\theta_{1}^{\infty}\langle 0\rangle\right)$ over $\widetilde{\xi}\langle 0\rangle$, respectively. Since $\theta_{1}^{\infty}\langle 0\rangle$ and $[\gamma]_{*}\left(\theta_{1}^{\infty}\langle 0\rangle\right)$ start from the same vanishing cycle $\sigma_{1}^{\infty}\langle 0\rangle$ by Claim 2 , we can choose $T$ and $T_{r}$ in such a way that their restrictions to the sub-path $\eta_{1}$ coincide ; 


$$
\left.T\right|_{\eta_{1}}=\left.T_{r}\right|_{\eta_{1}}
$$

(See Definition 4.5 for the definition of the restriction to a sub-path.) Let $T^{\prime}$ be the restriction of $T$ to the sub-path $\eta_{2}$, and $T_{r}^{\prime}$ the restriction of $T_{r}$ to the sub-path $\xi_{1}^{0}\langle 1\rangle \delta_{b}^{0}\langle 0\rangle \eta_{3}$. Then we have $\partial T^{\prime}=\partial T_{r}^{\prime}$, and hence we obtain an $n$-cycle

$$
T^{\prime \prime}:=T_{r}^{\prime}-T^{\prime}: C S^{n-1} \cup\left(-C S^{n-1}\right) \rightarrow F_{b}
$$

over the path $\xi_{1}^{0}\langle 1\rangle \delta_{b}^{0}\langle 0\rangle \eta_{3} \eta_{2}^{-1}$ from $p_{1}(b)\langle 0\rangle$ to $p_{1}(b)\langle 1\rangle$. Its homology class is

$$
\left[T^{\prime \prime}\right]=\left[T_{r}^{\prime}\right]-\left[T^{\prime}\right]=\left[T_{\gamma}\right]-[T]=[\gamma]_{*}\left(\bar{\theta}_{1}^{\infty}\langle 0\rangle\right)-\bar{\theta}_{1}^{\infty}\langle 0\rangle=v^{\prime} .
$$

Here we have used (9.4.19). This shows that $v^{\prime} \in H_{n}\left(F_{b}\right)$.

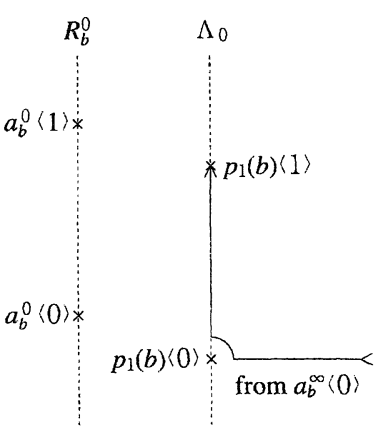

the path $\xi^{\prime}\langle 0\rangle$

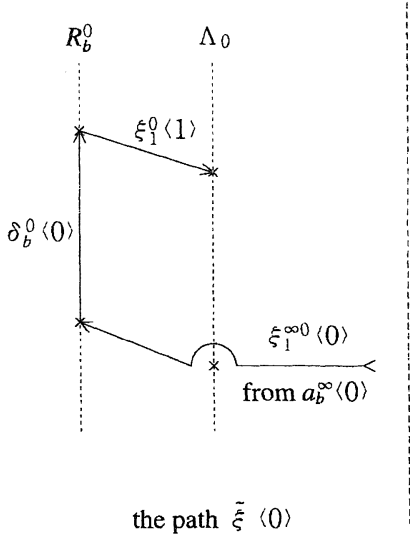

Figure 8

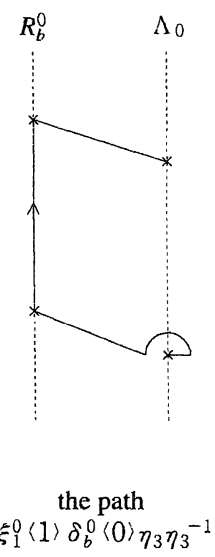

$\xi_{1}^{0}\langle 1\rangle \delta_{b}^{0}\langle 0\rangle \eta_{3} \eta_{3}{ }^{-1}$

The restriction $T_{(q)}$ of this $n^{\text {-cycle }} T^{\prime \prime}$ to the sub-path $\xi_{1}^{0}\langle 1\rangle$ represents a thimble for $\left[\xi_{1}^{0}\langle 1\rangle\right]$; that is, $\theta\left(\left[\xi_{1}^{0}\right]\right)\langle 1\rangle$ or $-\theta\left(\left[\xi_{1}^{0}\right]\right)\langle 1\rangle$. Hence its homology class is either $q v^{2}$ or $-q v^{2}$. Let $T_{(1)}$ be the restriction of $-T^{\prime \prime}$ to the sub-path $\eta_{2} \eta_{3}^{-1}$. Since $\left[\eta_{2} \eta_{3}^{-1}\right]=\left[\xi_{1}^{0}\langle 0\rangle\right]$ in $\left[\mathscr{P}_{b}^{\sim}\left(a_{b}^{0}\langle 0\rangle, p_{1}(b)\langle 0\rangle\right)\right], T_{(1)}$ represents a thimble for $\left[\xi_{1}^{0}\langle 0\rangle\right]$. Hence its homology class is either $v^{\circ}$ or $-v^{\circ}$. By the 
construction, $\partial T_{(1)} \subset X_{b}^{0}\langle 0\rangle$ and $\partial T_{(q)} \subset X_{b}^{0}\langle 1\rangle$ define a same homology class in $H_{n-1}\left(\phi_{b}^{-1}\left(R_{b}^{0}\right)\right)$. Let $\bar{\sigma}_{1}^{0}$ be the image of this homology class by the isomorphism $H_{n-1}\left(\phi_{b}^{-1}\left(R_{b}^{0}\right)\right) \cong H_{n-1}\left(X_{b}^{0}\right)$ induced from (6.18). Since the diffeomorphism (6.18) induces the isomorphism (6.1) over each point $a_{b}^{0}\langle\nu\rangle \in R_{b}^{0}$, Corollary 6.2 implies that $\left[T_{(1)}\right]=\Psi_{b}^{0}\left(\bar{\sigma}_{1}^{0} \otimes 1\right)$ and $\left[T_{(q)}\right]=\Psi_{b}^{0}\left(\bar{\sigma}_{1}^{0} \otimes q\right)$, and thus we have $\left[T_{(q)}\right]=q\left[T_{(1)}\right]$. Since the remaining part of $T^{\prime \prime}$ after deleting $T_{(q)}$ and $-T_{(1)}$ is contained in $\partial_{0} F_{b}$, we have

$$
\left[T^{\prime \prime}\right]=\left[T_{(q)}\right]-\left[T_{(1)}\right]= \pm(q-1) v^{\sim} \text { in } H_{n}\left(F_{b}, \partial_{0} F_{b}\right)
$$

The sign is determined by the condition (9.4.16) and Lemma 4.1.

As in Remarks 9.2 .2 and 9.2.3, we get the following:

Corollary 9.4.1. Let $\gamma$ and $\gamma^{\prime}$ be simple loops around $\mathscr{D}_{0}$ with the base point $b$ and $b^{\prime}$, respectively. Let $\alpha$ be a path from $b$ to $b^{\prime}$ in $U$ such that $[\alpha]^{-1}\left[\gamma^{\prime}\right][\alpha]=$ $[\gamma]$ holds in $\pi_{1}(\mathcal{U}, b)$. Then we have a congruence $\left(v\left[\gamma^{\prime}\right], v^{\sim}\left[\gamma^{\prime}\right]\right) \equiv[\alpha]_{*}(v[\gamma]$. $\left.v^{\sim}[\gamma]\right) \operatorname{modulo} U\left(\mathbb{Z}\left[q, q^{-1}\right]\right)$ in $H_{n}\left(F_{b^{\prime}}\right) \times H_{n}\left(F_{b^{\prime}}, \partial_{0} F_{b^{\prime}}\right)$.

\section{\$9.5. A Generator of $H_{n}\left(F_{b}\right)$ as m $\pi_{1}(U)$-Mlodule}

Let $\mathbb{Z}\left[q, q^{-1}\right]\left[\pi_{1}(U, b)\right]$ be the group ring of $\pi_{1}(U, b)$ with coefficients in $\mathbb{Z}\left[q, q^{-1}\right]$. We can consider $H_{n}\left(F_{b}\right), H_{n}\left(F_{b}, \partial_{0} F_{b}\right)$ and $H_{n}\left(F_{b}, \partial_{\infty} F_{b}\right)$ as modules over this ring in a natural way.

Theorem 9.5.1. Let $\gamma: I \rightarrow \mathcal{U}$ be a simple loop around $\mathscr{D}_{0}$ with the base point $b$. Then $v^{\sim}[\gamma]$ in Theorem 9.2 .1 generates the $\mathbb{Z}\left[q, q^{-1}\right]\left[\pi_{1}(\mathcal{U}, b)\right]$-module $H_{n}\left(F_{b}, \partial_{0} F_{b}\right)$, and $v[\gamma]$ generates the $\mathbb{Z}\left[q, q^{-1}\right]\left[\pi_{1}(\mathcal{U}, b)\right]$-module $H_{n}\left(F_{b}\right)$.

Before proving this theorem, we need some preparation.

Definition 9.5.1. We define $U_{N}^{*} \subset \mathcal{U}_{N}^{\tilde{N}}$ to be the locus of all $u \in \mathcal{U}_{N}$ such that, if $p_{l}$ and $p_{j}$ are distinct values in $\operatorname{Cr}(u)$, then $\left|\arg p_{l}-\arg p_{j}\right|$ is not 0 nor $\pi$.

It is obvious that $\Gamma \backslash \mathcal{U}_{N}^{*}$ is a real semi-algebraic subset of real codimension $\geq 1$.

Lemma 9.5.1. Let $b$ be a point of $U_{N}^{*}$. We put $\operatorname{Cr}(b)=\left\{p_{1}, \ldots, p_{N}\right\}$. Let $\lambda_{i}^{0}$ : $I \rightarrow \mathbb{C}^{\times}$be the path given by $t \mapsto(1-t) r+t \cdot p_{l}$, where $r$ is the small positive real number chosen in Remark 9.3.1. Then $\lambda_{i}^{0}$ is an element of $\mathscr{P}_{b}\left(a_{b}^{0}, p_{i}\right)$. Moreo- 
ver, there exist paths $\xi_{i}^{0} \in \mathscr{P}_{b}\left(a_{b}^{0}, p_{i}\right)$ for $i=1, \ldots, N$ such that $\left[\xi_{i}^{0}\right]=\left[\lambda_{i}^{0}\right]$ in $\left[\mathscr{P}_{b}\left(a_{b}^{0}, p_{i}\right)\right]$ for each $i$, and that $\left\{\xi_{1}^{0}, \ldots, \xi_{N}^{0}\right\}$ is a $K$-regular system of paths from $a_{b}^{0}$.

Proof. Note that we have $a_{b}^{0}=r$ by Remark 9.3.1. By the definition of $\mathcal{U}_{N}^{*}$, the path $t \mapsto t \cdot p_{\imath}$ on $\mathbb{C}$ from 0 to $p_{i}$ does not pass through any critical values of $\widehat{\phi}_{b}$ other than $p_{l}$. Since $r$ is small enough, $\lambda_{\imath}^{0}$ is also disjoint from $\operatorname{Cr}(b) \backslash\left\{p_{l}\right\}$. Hence $\lambda_{\imath}^{0} \in \mathscr{P}_{b}\left(a_{b}^{0}, p_{i}\right)$. We put

$$
K_{b}^{*}:=K_{b} \cup\{z \in \mathbb{C} ;|z| \leq r, \text { and }-\pi+\eta(b) / 2 \leq \arg (z-r) \leq \pi-\eta(b) / 2\},
$$

where $\eta$ is the function defined by (6.2). Then each $\lambda_{i}^{0}$ is contained in $K_{b}^{*}$. It is easy to see that there is a homotopy of continuous maps $\left\{g_{t}: K_{b}^{*} \rightarrow K_{b}^{*}\right\}_{t \in I}$ which satisfies the following: (i) $g_{0}$ is the identity, (ii) $g_{1}\left(K_{b}^{*}\right) \subset K_{b}$, (iii) $g_{t}$ is a homeomorphism onto its image for all $t \in I$, and (iv) $g_{t}\left(p_{i}\right)=p_{l}(i=1, \ldots, N)$ and $g_{t}\left(a_{b}^{0}\right)=a_{b}^{0}$ for all $t \in I$. We put $\xi_{l}^{0}:=g_{1} \circ \lambda_{l}^{0}$. It is obvious that $\left[\xi_{i}^{0}\right]=$ $\left[\lambda_{l}^{0}\right]$ in $\left[\mathscr{P}_{b}\left(a_{b}^{0}, p_{l}\right)\right]$, and that $\left\{\xi_{1}^{0}, \ldots, \xi_{N}^{0}\right\}$ is a $K$-regular system of paths from $a_{b}^{0}$.
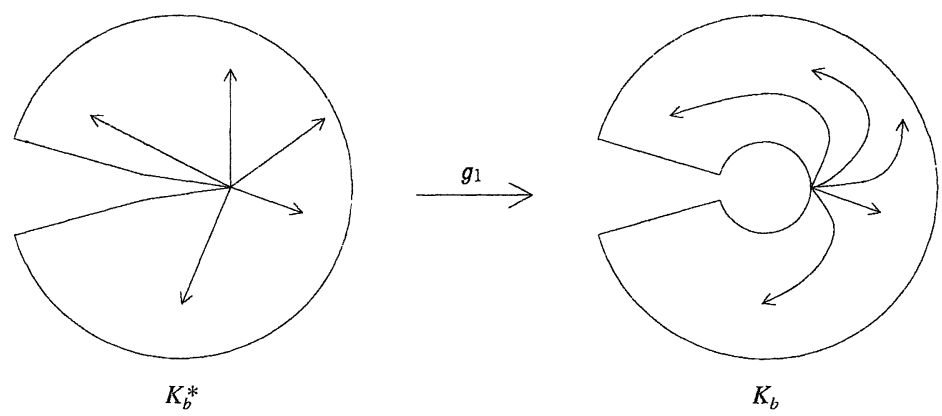

the paths $\left\{\lambda_{1}^{0}, \ldots, \lambda_{N}^{0}\right\}$ and $\left\{\xi_{1}^{0}, \ldots, \xi_{N}^{0}\right\}$

Figure 9

Now Theorem 9.5.1 follows from the following proposition whose proof will be given later. This proposition also plays an important role in the proof of Irreducibility Theorem.

Proposition 9.5.1. Suppose that $b \in \mathcal{U}_{N}^{*}$. Let $p$ be a value in $\mathrm{Cr}(b)$, and let $\lambda^{0}: I \rightarrow \mathbb{C}$ be the path from $a_{b}^{0}=r$ to $p$ given by $t \mapsto(1-t) r+t \cdot p$. Then there exists a simple loop $\gamma_{0}$ around $\mathscr{D}_{0}$ with the base point $b$ such that $v^{\sim}\left[\gamma_{0}\right] \equiv$ $\bar{\theta}\left(\left[\lambda^{0}\right]\right)\langle 0\rangle$ in $H_{n}\left(F_{b}, \partial_{0} F_{b}\right)$. 
Proof of Theorem 9.5.1. Since $v[\gamma]= \pm(1-q) v^{\sim}[\gamma]$ and $H_{n}\left(F_{b}\right)=(1-q) H_{n}$ $\left(F_{b}, \partial_{0} F_{b}\right)$, the second assertion follows from the first.

It is enough to prove this theorem under the assumption that $b \in U_{N}^{*}$. We put $\operatorname{Cr}(b)=\left\{p_{1}, \ldots, p_{N}\right\}$, and let $\lambda_{i}^{0}: I \rightarrow \mathbb{C}$ denote the path given by $t \mapsto(1-t) r$ $+t \cdot p_{i}$. By Lemma 9.5.1, there exists a $K$-regular system $\left\{\xi_{1}^{0}, \ldots, \xi_{N}^{n}\right\}$ of paths from $a_{b}^{0}=r$ such that $\left[\xi_{i}^{0}\right]=\left[\lambda_{i}^{0}\right]$ in $\left[\mathscr{P}_{b}\left(a_{b}^{0}, p_{i}\right)\right]$. In particular, we have $\bar{\theta}\left(\left[\xi_{i}^{0}\right]\right)\langle 0\rangle= \pm \bar{\theta}\left(\left[\lambda_{i}^{0}\right]\right)\langle 0\rangle$ in $H_{n}\left(F_{b}, \partial_{0} F_{b}\right)$ for $i=1, \ldots, N$. By Proposition 7.3 , we see that $\bar{\theta}\left(\left[\xi_{1}^{0}\right]\right)\langle 0\rangle, \ldots, \bar{\theta}\left(\left[\xi_{N}^{0}\right]\right)\langle 0\rangle$ generate $H_{n}\left(F_{b}, \partial_{0} F_{b}\right)$ as a $\mathbb{Z}\left[q, q^{-1}\right]$-module. By Proposition 9.5.1, there exist simple loops $\gamma_{1}, \ldots, \gamma_{N}$ around $\mathscr{D}_{0}$ with the base point $b$ such that $v^{\vee}\left[\gamma_{i}\right] \equiv \bar{\theta}\left[\xi_{i}^{0}\right]\langle 0\rangle$ for $i=1, \ldots, N$. Hence $v^{\vee}\left[\gamma_{1}\right], \ldots, v^{\vee}\left[\gamma_{N}\right]$ generate $H_{n}\left(F_{b}, \partial_{0} F_{b}\right)$ as a $\mathbb{Z}\left[q, q^{-1}\right]$-module. On the other hand, by Proposition 9.1.1, there exists $\left[\alpha_{i}\right] \in \pi_{1}(\mathcal{U}, b)$ for each $i$ such that $\left[\alpha_{i}\right]^{-1}\left[\gamma_{i}\right]\left[\alpha_{i}\right]=[\gamma]$, where $\gamma$ is the simple loop around $\mathscr{D}_{0}$ given in the statement of Theorem 9.5.1. By Corollary 9.4.1, we have $v^{\sim}\left[\gamma_{i}\right] \equiv\left[\alpha_{i}\right] * v^{\sim}[\gamma]$. Hence $v^{\bullet}[\gamma]$ generates $H_{n}\left(F_{b}, \partial_{0} F_{b}\right)$ as a $\mathbb{Z}\left[q, q^{-1}\right]\left[\pi_{1}(\mathcal{U}, b)\right]$-module.

Proof of Proposition 9.5.1. We use the following notation: for two values $w, z$ $\in \mathbb{C}$, we denote by $\lambda[w, z]: I \rightarrow \mathbb{C}$ the path from $z$ to $w$ given by $t \mapsto(1-t) z$ $+t w$, and by $\Lambda[w, z]$ its image $\lambda[w, z](I) \subset \mathbb{C}$.

Let $\left\{c_{1}, \ldots, c_{N}\right\}$ be the intersection points of $\mathscr{L}_{b}$ and $\mathscr{D}_{0}$. For $u \in \mathscr{L}_{b}$, we put $p_{\imath}(u):=\iota_{u}^{-1}\left(c_{\imath}\right)$. We have $\operatorname{Cr}(u)=\left\{p_{1}(u), \ldots, p_{N}(u)\right\}$ by Corollary 2.1. By renumbering $c_{1}, \ldots, c_{N}$, we assume that the point $p \in \mathrm{Cr}(b)$ given in the statement of Proposition 9.5.1 is $p_{1}(b)$.

Since $b \in U_{N}^{*}$, we have

$$
\Lambda\left[p_{1}(b), 0\right] \cap \operatorname{Cr}(b)=\left\{p_{1}(b)\right\}
$$

Let $\Delta_{1} \subset \mathbb{C}$ denote a sufficiently small closed disk with the center $p_{1}(b)$. (However, the number $r$ in Remark 9.3.1 is small enough compared with the radius of $\Delta_{1}$.) Since $\Delta_{1}$ is small enough, (9.5.1) implies that there exists a point $p_{1}(b)-\rho$ on the boundary $\partial \Delta_{1}$ such that

$$
\Lambda\left[p_{1}(b)-\rho, 0\right] \cap \Delta_{1}=\left\{p_{1}(b)-\rho\right\} \text { and } \Lambda\left[p_{1}(b)-\rho, 0\right] \cap \operatorname{Cr}(b)=\varnothing .
$$

Moreover, we may assume that 


$$
\begin{array}{ll}
\operatorname{Im} \rho>0 & \text { if } \operatorname{Im} p_{1}(b)>0, \\
\operatorname{Im} \rho=0 \text { and } \operatorname{Re} \rho>0 & \text { if } \operatorname{Im} p_{1}(b)=0, \text { and } \\
\operatorname{Im} \rho<0 & \text { if } \operatorname{Im} p_{1}(b)<0 .
\end{array}
$$

(Note that if $\operatorname{Im} p_{1}(b)=0$, then Re $p_{1}(b)>0$ because of $b \in U_{N}$.) We put

$$
b^{\prime}:=\iota_{b}\left(p_{1}(b)-\rho\right)
$$

and let $\gamma_{0}^{\prime}: I \rightarrow \mathscr{L}_{b}$ be a counter-clockwise loop along $\iota_{b}\left(\partial \Delta_{1}\right)$ with the base point $b^{\prime}$. Since $\mathscr{L}_{b}$ intersects $\mathscr{D}_{0}$ transversely by Proposition 2.4 , and $|\rho|$ is sufficiently small, $\gamma_{0}^{\prime}$ is a simple loop around $\mathscr{D}_{0}$. Since $|\rho|$ is small enough and $b \in U_{N}^{*}$, (9.5.3) implies that none of $p_{1}(b), \ldots, p_{N}(b)$ is on the horizontal semi-line $p_{1}(b)-\rho+\mathbf{R}_{\leq 0}$. Hence, by Lemma 9.4.1, we have

$$
b^{\prime} \in \mathcal{U}_{N}^{\tilde{n}}
$$

so that we can use Proposition 9.4.1 for the local monodromy $\left[\gamma_{0}^{\prime}\right]_{*}$ around $\mathscr{D}_{0}$. We have $p_{1}\left(b^{\prime}\right)=\rho$ by Lemma 9.4.1. Let $D_{1}^{\prime} \subset \mathbb{C}$ be the closed disk with the center 0 and of radius $|\rho|$. Since $|\rho|$ is small enough, we have $D_{1}^{\prime} \cap \operatorname{Cr}\left(b^{\prime}\right)=$ $\left\{p_{1}\left(b^{\prime}\right)\right\}$. We also have $\varepsilon\left(b^{\prime}\right)=r$ by Remark 9.3.1. Therefore, the homotopy class $[\lambda[\rho, v]] \in\left[\mathscr{P}_{b^{\prime}}\left(a_{b^{\prime}}^{0}, p_{1}\left(b^{\prime}\right)\right)\right]$ of the straight path $\lambda[\rho, v]$ from $a_{b^{\prime}}^{0}=r$ to $p_{1}\left(b^{\prime}\right)=\rho$ is represented by a path $\xi_{1}^{0}$ which is contained in $K_{b^{\prime}} \cap D_{1}^{\prime}$. Hence $[\lambda[\rho, r]]=\left[\xi_{1}^{0}\right]$ is the unique homotopy class in $\left[\mathscr{P}_{b^{\prime}}\left(a_{b^{\prime}}^{0}, p_{1}\left(b^{\prime}\right)\right)\right]$ characterized by (9.4.8). Using Proposition 9.4.1, we have

$$
v^{\sim}\left[\gamma_{0}^{\prime}\right] \equiv \bar{\theta}([\lambda[\rho, \gamma]])\langle 0\rangle \quad \text { in } \quad H_{n}\left(F_{b^{\prime}}, \partial_{0} F_{b^{\prime}}\right) .
$$

Let $\beta$ be a path on $\mathscr{L}_{b}$ from $b^{\prime}$ to $b$ given by

$$
\beta:=\iota_{b} \circ \lambda\left[0, p_{1}(b)-\rho\right] .
$$

By (9.5.2), this path does not pass through any point of $\iota_{b}(\mathrm{Cr}(b))=\mathscr{L}_{b} \cap \mathscr{D}_{0}$, and hence it is a path in $U$ by Corollary 2.1. Note that $a_{\beta(t)}^{0}=r$ for all $t \in I$ because of Remark 9.3.1. In particular, we have $a_{b^{\prime}}^{0}=r$. We put

$$
\gamma_{0}:=\beta \gamma_{0}^{\prime} \beta^{-1}
$$

Since $\gamma_{0}^{\prime}$ is a simple loop around $\mathscr{D}_{0}$, so is the loop $\gamma_{0}$. We shall show that this $\gamma_{0}$ is the desired loop; that is, $v^{v}\left[\gamma_{0}\right]$ is congruent with $\bar{\theta}\left(\left[\lambda^{0}\right]\right)\langle 0\rangle$ in $H_{n}\left(F_{b}\right.$, 
$\left.\partial_{0} F_{b}\right)$ modulo $U\left(\mathbb{Z}\left[q, q^{-1}\right]\right)$. By Corollary 9.4.1, we have

$$
v^{\sim}\left[\gamma_{0}\right] \equiv[\beta]_{*}\left(v^{\sim}\left[\gamma_{0}^{\prime}\right]\right) \text { in } H_{n}\left(F_{b}, \partial_{0} F_{b}\right)
$$

By (9.5.5) and (9.5.6), it is enough to prove

$$
\bar{\theta}\left(\left[\lambda^{0}\right]\right)\langle 0\rangle= \pm[\beta]_{*}(\bar{\theta}([\lambda[\rho, r]])\langle 0\rangle) \text { in } H_{n}\left(F_{b}, \partial_{0} F_{b}\right) \text {. }
$$

In order to prove this, we will study the bijective map

$$
[\beta]_{*}:\left[\left(C S^{n-1}, S^{n-1}\right),\left(F_{b^{\prime}}, X_{b^{\prime}}^{0}\langle 0\rangle\right)\right] \stackrel{\sim}{\rightarrow}\left[\left(C S^{n-1}, S^{n-1}\right),\left(F_{b}, X_{b}^{0}\langle 0\rangle\right)\right]
$$

By Lemma 2.3, we have $\mathscr{L}_{b} \backslash \mathscr{D}_{0} \subset \mathscr{U}_{N}$. Hence we have a map $[\beta]_{*}: \mathscr{C}_{r}\left(b^{\prime}\right)$ $\rightarrow \mathscr{C} r(b)$. The value $p_{1}(\beta(t))$ draws a straight path $\lambda\left[p_{1}(b), \rho\right]$ on $\mathbb{C}$ by Lemma 9.4.1. Because of the assumption (9.5.3), this path does not traverse $\mathbb{R}_{\leq 0}$. Hence we have

$$
[\beta]_{*}\left(p_{1}\left(b^{\prime}\right)\langle\nu\rangle\right)=p_{1}(b)\langle\nu\rangle \text { for all } \nu \in \mathbb{Z}
$$

From (9.5.9), we obtain the following commutative diagram :

$$
\begin{array}{ccc}
{\left[\mathscr{P}_{b^{\prime}}^{\sim}\left(a_{b^{\prime}}^{0}\langle 0\rangle, p_{1}\left(b^{\prime}\right)\langle 0\rangle\right)\right]} & \underset{[\beta]_{\tilde{*}}}{\sim} & {\left[\mathscr{P}_{b}^{\sim}\left(a_{b}^{0}\langle 0\rangle, p_{1}(b)\langle 0\rangle\right)\right]} \\
& \underset{\left.\mathscr{P}_{b^{\prime}}\left(a_{b^{\prime}}^{0}, p_{1}\left(b^{\prime}\right)\right)\right]}{\stackrel{\sim}{\longrightarrow}} & {\left[\mathscr{P}_{b}\left(a_{b}^{0}, p_{1}(b)\right)\right],}
\end{array}
$$

where the vertical hook-arrows are the injective maps in Proposition 7.1. Because both of $\Lambda[\rho, r]$ and $\lambda^{0}(I)=\Lambda\left[p_{1}(b), r\right]$ are contained in $\mathbb{C} \backslash \mathbb{R} \leq 0$, we have $\lambda[\rho, r]\langle 0\rangle(1)=p_{1}\left(b^{\prime}\right)\langle 0\rangle$ and $\lambda^{0}\langle 0\rangle(1)=p_{1}(b)\langle 0\rangle$; that is,

$$
[\lambda[\rho, r]\langle 0\rangle] \in\left[\mathscr{P}_{b^{\prime}}^{\sim}\left(a_{b^{\prime}}^{0}\langle 0\rangle, p_{1}\left(b^{\prime}\right)\langle 0\rangle\right)\right] \text {, and }
$$

$$
\left[\lambda^{0}\langle 0\rangle\right] \in\left[\mathscr{P}_{b}^{\sim}\left(a_{b}^{0}\langle 0\rangle, p_{1}(b)\langle 0\rangle\right)\right]
$$

In order to to prove (9.5.7), it is enough to show that

$$
[\beta]_{*}([\lambda[\rho, r]])=\left[\lambda^{0}\right] \text { in }\left[\mathscr{P}_{b}\left(a_{b}^{0}, p_{1}(b)\right)\right]
$$

Indeed, by the commutative diagram ('9.5.10) and the first formula of (9.5.11), (9.5.12) will imply that $[\beta]_{*}^{\sim}([\lambda[\rho, r]\langle 0\rangle])$ is an element of $\left[\mathscr{P}_{b}^{\sim}\left(a_{b}^{0}\langle 0\rangle\right.\right.$, $\left.\left.p_{1}(b)\langle 0\rangle\right)\right]$ whose projection in $\left[\mathscr{P}_{b}\left(a_{b}^{0}, p_{1}(b)\right)\right]$ is $\left[\lambda^{0}\right]$. Hence, by the second 
formula of (9.5.11), we obtain

$$
[\beta]_{*}^{\sim}([\lambda[\rho, r]\langle 0\rangle])=\left[\lambda^{0}\langle 0\rangle\right] \text { in }\left[\mathscr{P}_{b}^{\sim}\left(a_{b}^{0}\langle 0\rangle, p_{1}(b)\langle 0\rangle\right)\right]
$$

Since $\theta\left(\left[\lambda^{0}\right]\right)\langle 0\rangle$ is a thimble for $\left[\lambda^{0}\langle 0\rangle\right]$ and $\theta([\lambda[\rho, r]])\langle 0\rangle$ is a thimble for $[\lambda[\rho, r]\langle 0\rangle],(9.5 .13)$ implies that the bijection $[\beta] *$ in $(9.5 .8)$ maps $\theta([\lambda[\rho$, r] $])\langle 0\rangle$ to $\theta\left(\left[\lambda^{0}\right]\right)\langle 0\rangle$ or $-\theta\left(\left[\lambda^{0}\right]\right)\langle 0\rangle$. This implies $(9.5 .7)$.

Now we shall prove (9.5.12). By Lemma 9.4.1, we see that $p_{i}(\beta(t))$ draws the path $\lambda\left[p_{i}(b), p_{i}\left(b^{\prime}\right)\right]$, and that $p_{i}\left(b^{\prime}\right)$ is given by $p_{i}\left(b^{\prime}\right)=p_{i}(b)-p_{1}(b)+\rho$. The track of the movement of the ending point $p_{1}\left(b^{\prime}\right)=\rho$ of $\lambda[\rho, r]$ is given by $\Lambda\left[p_{1}(b), \rho\right]$. We shall see that

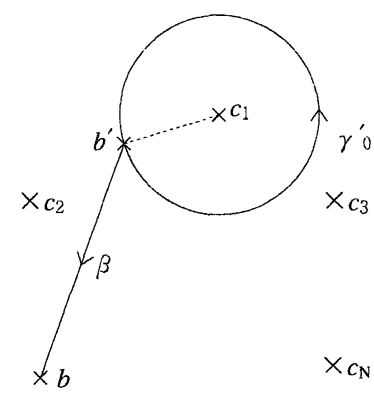

on $\mathscr{L}_{b}$

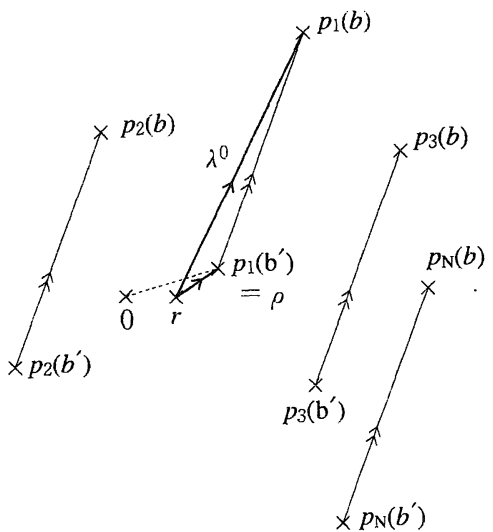

on $\mathbf{C}$

Figure 10

$$
\left(\Lambda\left[p_{1}(b), \rho\right] \cup \Lambda[\rho, r]\right) \cap \Lambda\left[p_{i}(b), p_{i}\left(b^{\prime}\right)\right]=\emptyset \text { if } i \neq 1
$$

Indeed, the two line segments $\Lambda\left[p_{1}(b), 0\right]$ and $\Lambda\left[p_{i}(b), p_{i}(b)-p_{1}(b)\right]$ are parallel, but, if $i \neq 1$, they are not on the same line because of $b \in U_{N}^{*}$. Hence they are disjoint. Since $r$ and $\rho$ are small enough, we see that $\left(\Lambda\left[p_{1}(b), \rho\right] \cup\right.$ $\Lambda[\rho, r])$ and $\Lambda\left[p_{i}(b), p_{i}(b)-p_{1}(b)+\rho\right]$ are still disjoint if $i \neq 1$. Hence (9.5.14) holds. This implies that the path $\lambda[\rho, r]$ stretches to $\lambda\left[p_{1}(b), \rho\right]$. $\lambda[\rho, r]$ by the movement of the ending point $p_{1}(\beta(t))$ of the path without being affected by the movement of any other points $p_{2}(\beta(t)), \ldots, p_{N}(\beta(t))$, and with the 
starting point fixed by Remark 9.3.1. Thus we have

$$
[\beta]_{*}([\lambda[\rho, r]])=\left[\lambda\left[p_{1}(b), \rho\right] \cdot \lambda[\rho, r]\right] .
$$

It is easy to see from (9.5.3) that the triangle (or the line segment if it degenerates) spanned by the three points $p_{1}(b), \rho$ and $r$ does not contain 0 . Moreover, since $r$ and $\rho$ are sufficiently small, (9.5.1) implies that this triangle does not contain any points of $\mathrm{Cr}(b) \backslash\left\{p_{1}(b)\right\}$. Hence we have

$$
\left[\lambda\left[p_{1}(b), \rho\right] \cdot \lambda[\rho, r]\right]=\left[\lambda\left[p_{1}(b), r\right]\right]=\left[\lambda^{0}\right] \text { in }\left[\mathscr{P}_{b}\left(a_{b}^{0}, p_{1}(b)\right)\right]
$$

Combining this with (9.5.15), we get (9.5.12).

\section{\$9.6. The Behavior of $\mathrm{Cr}(u)$ mear $\mathscr{D}_{\infty}$}

In this subsection, we shall investigate how the set of the critical values $\operatorname{Cr}(u)$ of $\widehat{\phi}_{u}$ behaves when $u$ approaches a point of $\mathscr{D}_{\infty}$. The result will be used in the proof of Theorem 9.2.2.

We choose a general affine line $\mathscr{A}$ in $\Gamma$. Let $c$ be an intersection point of $\mathscr{A}$ and $\mathscr{D}_{\infty}$. Since $\mathscr{A}$ is general, $c$ is a non-singular point of $\mathscr{D}_{\infty} \backslash\left(\mathscr{D}_{\infty} \cap \mathscr{D}_{0}\right)$, and the intersection of $\mathscr{A}$ and $\mathscr{D}_{\infty}$ is transverse at $c$. Let $\Delta$ be a sufficiently small closed disk on $\mathscr{A}$ with the center $c$. We choose a base point $b$ on the boundary $\partial \Delta$, and let $\gamma: I \rightarrow \mathscr{A}$ denote the counter-clockwise loop with the base point $b$ along $\partial \Delta$. Since $\Delta$ is small enough, $\gamma$ is a simple loop around $\mathscr{D}_{\infty}$. We shall say that $\gamma$ is a simple loop around $\mathscr{D}_{\infty}$ associated with the data $(\mathscr{A}, c, b, \Delta)$. Since $\mathscr{A}$ is general and $\Delta$ is small, we may assume that

$$
\Delta \backslash\{c\} \subset \mathcal{U}_{N}
$$

Moreover, by choosing $b$ generally, we may also assume that

$$
b \in \mathcal{U}_{N}^{\sim}
$$

By (9.6.1), $[\gamma]_{*}$ acts on the set $\mathrm{Cr}(b)$.

Proposition 9.6.1. (1) The action of $[\gamma]_{*}$ on $\mathrm{Cr}(b)$ is trivial. In particular, there are holomorphic functions $p_{1}(u), \ldots, p_{N}(u)$ on $\Delta \backslash\{c\}$ such that

$$
\operatorname{Cr}(u)=\left\{p_{1}(u), \ldots, p_{N}(u)\right\} \quad \text { for } u \in \Delta \backslash\{c\} \text {. }
$$


(2) There exists one and only one function among $\left\{p_{1}(u), \ldots, p_{N}(u)\right\}$, say $p_{N}(u)$, which has a pole of order $d-1$ at $u=c$. (3) The other functions $p_{1}(u), \ldots, p_{N-1}(u)$ can be extended holomorphically over $u=c$. (4) The values $p_{1}(c), \ldots, p_{N-1}(c)$ are distinct to each other and different from 0 .

Proof. Since $\mathscr{A}$ is general and $\Delta$ is small enough, we have the following: $(\mathrm{x}-1) \bar{X}_{u}$ is non-singular for all $u \in \Delta$,

$(\mathrm{x}-2) \bar{X}_{c}$ is tangent to $H_{\infty}$ at a point $P$,

$(\mathrm{x}-3) \bar{X}_{c} \cap H_{\infty}$ has an ordinary double point at $P$ as its only singularities, and $(\mathrm{x}-4)$ if $u \in \Delta \backslash\{c\}$, then $\bar{X}_{u} \cap H_{\infty}$ is non-singular.

We apply Construction 2.1 to our data $(\mathscr{A}, c, b, \Delta)$ and obtain the finite covering $\rho: C \rightarrow \mathscr{A}$ with morphisms $\tilde{q}_{t}: C \rightarrow \mathbb{P}^{n}=\mathbf{A}^{n} \cup H_{\infty}$ and $\tilde{p}_{i}: C \rightarrow \mathbb{P}^{1}=\mathbb{C} \cup$ $\{\infty\}$.

Claim 1. There is one and only one morphism among $\left\{\tilde{q}_{1}, \ldots, \tilde{q}_{N}\right\}$, say $\tilde{q}_{N}$, such that $\tilde{q}_{N}(\tilde{c})$ is contained in $H_{\infty}$. Moreover we have $\tilde{q}_{N}(\tilde{c})=P$.

Proof. As in Construction 2.2, we choose an affine subspace $\left(\mathbf{A}^{n}\right)^{\prime}$ of $\mathbf{P}^{n}$ which contains $\tilde{q}_{1}(\tilde{c}), \ldots, \tilde{q}_{N}(\tilde{c})$ and moreover, the point $P$. Let $\left(z_{1}, \ldots, z_{n}\right)$ be affine coordinates on $\left(\mathbb{A}^{n}\right)^{\prime}$ such that

$$
H_{\infty}=\left\{z_{n}=0\right\}, \text { and } P=(0, \ldots, 0) .
$$

Recall that there exist an affine coordinate $t: \mathscr{A} \rightarrow \mathbf{C}$ with $t(c)=0$ and a homogeneous polynomial $g \in \Gamma^{\times}$such that $f_{u}$ is equal with $f_{c}+t(u) \cdot g$ for $u \in$ $\Delta$. (See (2.6) .) We choose inhomogeneous polynomials $f_{c}\left(z_{1}, \ldots, z_{n}\right)$ and $g\left(z_{1}, \ldots, z_{n}\right)$ associated to $f_{c}$ and $g$, respectively, such that the rational function $\bar{\phi}_{u}$ $=f_{u} / h^{d}$ on $\left(\mathbb{A}^{n}\right)^{\prime}$ can be written in the form $(2.7)$ for $u \in \Delta$. Let $f_{u}^{[\nu]}\left(z_{1}, \ldots, z_{n}\right)$ denote the homogeneous part of degree $\nu$ of

$$
f_{u}\left(z_{1}, \ldots, z_{n}\right)=f_{c}\left(z_{1}, \ldots, z_{n}\right)+t(u) \cdot g\left(z_{1}, \ldots, z_{n}\right)
$$

Then the properties $(x-1)-(x-3)$ imply that

$\left(\mathrm{fc}_{\mathrm{c}}-1\right) f_{c}^{[0]}=0$

$(\mathrm{fc}-2) f_{c}^{[1]}=a z_{n}$, where $a$ is a non-zero constant, and

(fc-3) $f_{c}^{[2]}\left(z_{1}, \ldots, z_{n-1}, 0\right)$ is a non-degenerate quadratic form in $z_{1}, \ldots, z_{n-1}$.

Recall the definition of the polynomials $h_{1}\left(u ; z_{1}, \ldots, z_{n}\right), \ldots, h_{n}\left(u ; z_{1}, \ldots, z_{n}\right)$ in Construction 2.2. By $(\mathrm{fc}-1)$ and $(\mathrm{fc}-2)$, we see that 
$\frac{\partial h_{n}}{\partial z_{i}}(c ; 0, \ldots, 0)=0$ for $i=1, \ldots, n-1 \quad$ and $\quad \frac{\partial h_{n}}{\partial z_{n}}(c ; 0, \ldots, 0)=(1-d) a \neq 0$

Combining these with $\left(\mathrm{fc}_{\mathrm{c}}-3\right)$, we obtain the following :

$$
\operatorname{det}\left[\frac{\partial h_{2}}{\partial z_{\jmath}}(c ; 0, \ldots, 0)\right]_{\imath, j=1, \cdots, n} \neq 0
$$

Recall also that $I(u)$ is defined to be the intersection of the hypersurfaces $H_{i}(u)$ defined by $h_{\imath}\left(u ; z_{1}, \ldots, z_{n}\right)=0$. We will prove the following three assertions:

Sub-claim $\mathbb{1} ; I(c) \cap H_{\infty}$ consists of only one point $P$, Sub-cldim 2 ; if $u \in \Delta \backslash\{c\}$, then $I(u) \cap H_{\infty}=\emptyset$, and

Sub-claim 3 ; each of $H_{1}(c), \ldots, H_{n}(c)$ is non-singular at $P$ and they intersect transversely at $P$.

Indeed, the coordinates of a point in $I(u) \cap H_{\infty}$ are the solution of

$$
\begin{gathered}
z_{n}=f_{u}\left(z_{1}, \ldots, z_{n-1}, 0\right)=0 \text {, and } \\
\frac{\partial f_{u}\left(z_{1}, \ldots, z_{n-1}, 0\right)}{\partial z_{\imath}}=0 \text { for } i=1, \ldots, n-1 .
\end{gathered}
$$

Since $f_{u}\left(z_{1}, \ldots, z_{n-1}, 0\right)=0$ defines the hypersurface $\bar{X}_{u} \cap H_{\infty}$ on $H_{\infty}$, the solution must be the coordinates of a singular point of $\bar{X}_{u} \cap H_{\infty}$. Hence the properties $(x-3)$ and $(x-4)$ imply Sub-claims 1 and 2, respectively. Sub-claim 3 follows from the non-degeneracy (9.6.4) of the Jacobian matrix of the defining equations of the hypersurfaces $H_{i}(c)$ at $P$.

By (2.9) and Sub-claim 2, we have

$$
I(\rho(w))=\left\{\tilde{q}_{1}(w), \ldots, \tilde{q}_{N}(w)\right\} \quad \text { for all } w \in \tilde{\Delta} \backslash\{\tilde{c}\}
$$

Let $V \subset\left(\mathbb{A}^{n}\right)^{\prime}$ be a small open neighborhood of $H_{\infty} \cap\left(\mathbb{A}^{n}\right)^{\prime}$. Then Sub-claims 1 and 3 imply that, if $u \in \Delta$, then $V \cap I(u)$ consists of only one point, say $P(u)$, such that $P(c)=P$; that is, the intersection point $P=P(c)$ of the hypersurfaces $H_{\imath}(c) \quad(i=1, \ldots, n)$ does not vanish nor split into plural points when $u$ moves away from $c$. If $w \neq \tilde{c}$, then $P(\rho(w))$ must be among $\left\{\tilde{q}_{1}(w), \ldots, \tilde{q}_{N}(w)\right\}$ because of (9.6.5). Putting $\tilde{q}_{N}(w)=P(\rho(w))$, we have proved Claim 1 .

Claim 1 implies that the morphism from $\mathscr{A} \cap \mathcal{U}_{N}$ to $\mathbb{A}^{n}$ corresponding to the critical point $q_{N}(b)$ is single-valued on $\Delta \backslash\{c\}$; that is, if we define $q_{N}^{\prime \prime}: \Delta$ $\rightarrow \mathbb{P}^{n}$ by $q_{N}^{\prime \prime}(u)=P(u)$, then $\tilde{q}_{N}\left|\tilde{\Delta}=\rho^{*} q_{N}^{\prime \prime}\right| \tilde{\Delta}$. Therefore, the corresponding 
critical value is also single-valued on $\Delta \backslash\{c\}$. In particular, $p_{N}(b) \in \mathrm{Cr}(b)$ is not changed under the action of $[\gamma]_{*}$ on $\mathrm{Cr}(b)$. Let $p_{N}^{\prime \prime}: \Delta \backslash\{c\} \rightarrow \mathrm{C}$ denote the corresponding critical value ; that is, $p_{N}^{\prime \prime}(u):=\widehat{\phi}_{u}\left(q_{N}^{\prime \prime}(u)\right)$. Then we have $\tilde{p}_{N}\left|\tilde{\Delta} \backslash\{\tilde{c}\}=\rho^{*} p_{N}^{\prime \prime}\right| \tilde{\Delta} \backslash\{\tilde{c}\}$.

Claim 2. The function $p_{N}^{\prime \prime}$ has a pole of order $d-1$ at $u=c$.

Proof. Let $\left(\zeta_{1}(u), \ldots, \zeta_{n}(u)\right)$ denote the coordinates of the point $q_{N}^{\prime \prime}(u)=$ $P(u)$ in terms of $\left(z_{1}, \ldots, z_{n}\right)$ above. Since $q_{N}^{\prime \prime}(c)=P(c)=P$ is the origin, we have

$$
\text { each } \zeta_{\imath}(u) \text { has a zero of order } \geq 1 \text { at } u=c \text {. }
$$

The function

$$
p_{N}^{\prime \prime}(u)=\widehat{\phi}_{u}\left(q_{N}^{\prime \prime}(u)\right)=\frac{f_{u}\left(\zeta_{1}(u), \ldots, \zeta_{n}(u)\right)}{\zeta_{n}(u)^{d}}
$$

has a pole of order $d-1$ at $u=c$ if the following holds :

(9.6.7) both of $\zeta_{n}(u)$ and $f_{u}\left(\zeta_{1}(u), \ldots, \zeta_{n}(u)\right)$ have a zero of order exactly 1 at $u=c$.

We put $\alpha_{n}:=\lim _{u \rightarrow c} \zeta_{n}(u) / t(u)$. By $(\mathrm{fc}-1),(\mathrm{fc}-2)$ and (9.6.6), we have

$$
\lim _{u \rightarrow c} \frac{f_{u}\left(\zeta_{1}(u), \ldots, \zeta_{n}(u)\right)}{t(u)}=a \alpha_{n}+g(0, \ldots, 0)
$$

Hence (9.6.7) is equivalent to the following:

$$
\alpha_{n} \neq 0, \quad \text { and } \quad a \alpha_{n}+g(0, \ldots, 0) \neq 0
$$

By (9.6.5), we see that $h_{n}\left(u ; \zeta_{1}(u), \ldots, \zeta_{n}(u)\right)$ is constantly equal with 0 for all $u$ $\in \Delta \backslash\{c\}$. Hence, by $\left(\mathrm{fc}_{\mathrm{c}}-1\right),(\mathrm{fc}-2)$ and (9.6.6) again, we obtain the following:

$$
\lim _{u \rightarrow c} \frac{h_{n}\left(u ; \zeta_{1}(u), \ldots, \zeta_{n}(u)\right)}{t(u)}=a \alpha_{n}-d\left(a \alpha_{n}+g(0, \ldots, 0)\right)=0
$$

Because $\mathscr{A}$ has been chosen generally, we can assume that $g(0, \ldots, 0) \neq 0$. Hence (9.6.9) implies the two inequalities in (9.6.8). Thus Claim 2 is proved. 
We define the subset $D_{0 \infty}$ of $D_{0} \cap D_{\infty}$ to be the locus of all $\bar{X} \in D_{0} \cap D_{\infty}$ such that $H_{\infty}$ is disjoint from the singular locus of $\bar{X}$. It is obvious that $D_{00}$ is a Zariski open subset of $D_{0} \cap D_{\infty}$. Recall that $L_{u}^{o} \subset \mathbb{P}_{*}(\Gamma)$ is the affine line $L_{u} \backslash\left\{\mathfrak{y}_{\infty}\right\}$. Suppose that $v$ is a point of $\mathscr{D}^{\infty}$. Then $L_{v}$ is a line in $D_{\infty}$ passing through $\mathfrak{y}_{\infty}$ by Proposition 2.2, and hence $L_{v}^{o} \cap D_{0} \subset D_{0} \cap D_{\infty}$. If $v \in \mathscr{D}_{\infty}$ is general, then $\bar{X}_{v}$ is non-singular and hence Lemma 2.1 implies that

$$
L_{v}^{o} \cap D_{0} \subset D_{0 \infty} \quad \text { for a general } v \in \mathscr{D}_{\infty} \text {. }
$$

We shall prove the following :

Clæim 3. The locus $D_{0 \infty}$ is irreducible.

Proof. Let $\left(\mathbb{P}^{n}\right)$ - denote the dual projective space of $\mathbb{P}^{n}$. For a singular projective hypersurface $\bar{X} \in D_{0}$, we put

$$
\begin{aligned}
\bar{X}^{*}: & =\left\{H \in\left(\mathbb{P}^{n}\right)^{\sim} ; H \text { is tangent to } \bar{X} \text { at its non-singular point }\right\}, \text { and } \\
\bar{X}^{* * *} & :=\left\{H \in \bar{X}^{*}: H \cap \text { Sing } \bar{X}=\emptyset\right\} .
\end{aligned}
$$

The dual hypersurface $\bar{X}$ of $\bar{X}$ is the closure of $\bar{X}^{*}$. If $\bar{X} \in D_{0}$ is general, then $\bar{X}$ has only one ordinary double point as its singularities. Hence, because of $d \geq 3, \bar{X}^{* *}$ is an irreducible locally Zariski closed subset of codimension 1 in $\left(\mathbb{P}^{n}\right)$. There are no $\bar{X} \in D_{0}$ such that $\bar{X}^{* *}$ is Zariski open dense in $\left(\mathbb{P}^{n}\right)$. We put

$$
\mathfrak{X}^{* * *}:=\left\{(\bar{X}, H) ; H \in \bar{X}^{* * *}\right\} \subset D_{0} \times\left(\mathbb{P}^{n}\right)-
$$

Since $D_{0}$ is irreducible, the above consideration implies that there exists only one irreducible component $\mathfrak{X}_{\text {max }}^{* * *}$ of $\mathfrak{X}^{* *}$ which is mapped dominantly onto $D_{0}$ by the first projection, and moreover, if there exists any other irreducible component $\mathfrak{X}_{1}^{* *}$ of $\mathfrak{x}^{* *}$, then we have

$$
\operatorname{dim} \mathfrak{X}_{1}^{* * *}<\operatorname{dim} \mathfrak{X}_{\text {max }}^{* *}
$$

Now consider the second projection

$$
\mathrm{pr}_{2}: \mathfrak{X}^{* * *} \rightarrow\left(\mathbb{P}^{n}\right) \text {. }
$$

This is a locally trivial fiber space in the sense of complex analytic geometry, 
because the automorphism group $P G L(n+1)$ of $\mathbf{P}^{n}$ acts on both of $\mathfrak{X}^{* * *}$ and $\left(\mathbf{P}^{n}\right)$ - in such a natural way that $\mathrm{pr}_{2}$ is equivariant, and because this action is transitive on $\left(\mathbf{P}^{n}\right)$. The space $D_{0 \infty}$ is nothing but the fiber of $\mathrm{pr}_{2}$ over $H_{\infty} \in$ $\left(\mathbf{P}^{n}\right)$. Since $D_{0 \infty}$ is Zariski open in $D_{0} \cap D_{\infty}$, every irreducible component of $D_{0 \infty}$ is of codimension 2 in $\mathbf{P}_{*}(\Gamma)$. Hence every irreducible component of $\mathfrak{X}^{* *}$ must have a same dimension. Combining this with (9.6.11), we see that $\mathfrak{X}^{* *}$ is irreducible. Therefore the fiber $D_{0 \infty}$ of $\operatorname{pr}_{2}$ must be irreducible because $\left(\mathbb{P}^{n}\right)$. is simply-connected. Thus Claim 3 is proved.

Claim 4. At every point of $L_{c}^{o} \cap D_{0}, D_{0}$ is non-singular, and $L_{c}^{o}$ intersects $D_{0}$ transversely.

Proof. Let $x$ be a point of $\mathbb{P}_{*}(\Gamma)$, and let $\tilde{x} \in \Gamma^{\times}$be a lifting of $x$. Since $L_{\tilde{x}}^{o} \subset \mathbb{P}_{*}(\Gamma)$ does not depend on the choice of $\tilde{x}$, we can denote it by $L_{x}^{o}$ instead of $L_{\tilde{x}}^{o}$. Consider the locus $\mathscr{G}$ of all points $x \in D_{\infty} \backslash\left\{\mathfrak{h}_{\infty}\right\}$ such that, at every point of $L_{x}^{o} \cap D_{0}, D_{0}$ is non-singular and $L_{x}^{o}$ intersects $D_{0}$ transversely. This locus $\mathscr{G}$ in obviously Zariski open in $D_{\infty} \backslash\left\{\mathfrak{h}_{\infty}\right\}$. By the generality of the position of $c$ in $\mathscr{D}_{\infty}$, it is enough to show that $\mathscr{G}$ is non-empty. Using (9.6.10) and Claim 3, we can reduce the claim $\mathscr{G} \neq \varnothing$ to the following : there exists at least one point $y$ $\in D_{0 \infty}$ such that $D_{0}$ is non-singular at $y$ and that $L_{y}^{0}$ intersects $D_{0}$ transversely at $y$.

Let $\operatorname{Ver}:\left(\mathbb{P}^{n}\right)^{\sim} \rightarrow \mathbb{P}_{*}(\Gamma)$ be the morphism given by $H \mapsto d \cdot H$. Note that $V e r$ is projectively equivalent to the Veronese embedding of degree $d$. Let $\bar{X}_{w}$ be the singular projective hypersurface corresponding to a general point $w$ of $D_{0}$, and let $\bar{X}_{w} \subset\left(\mathbb{P}^{n}\right)$ be the dual hypersurface of $\bar{X}_{w}$. Because $\bar{X}_{w}$ has one ordinary double point as its only singularities, and because of $d \geq 3$, we see that, for a general point $H \in \bar{X}_{w}$, the singular point of $\bar{X}_{w}$ is disjoint from $H$. Note also that the degree of $\bar{X}_{\tilde{w}}$ is $\geq d+1$ because of $d \geq 3$. Hence $\operatorname{Ver}\left(\bar{X}_{w}\right)$ is not contained in any hyperplane of $\mathbf{P}_{*}(\Gamma)$. Note that $D_{0}$ is non-singular at $w$ because $w$ is general in $D_{0}$. Let $T_{w} \subset \mathbb{P}_{*}(\Gamma)$ be the tangent hyperplane of $D_{0}$ at $w$. Then $\operatorname{Ver}\left(\bar{X}_{w}\right) \cap T_{w}$ is of codimension 1 in $\operatorname{Ver}\left(\bar{X}_{w}\right)$. Hence there exists a hyperplane $H_{1} \in \bar{X}_{\tilde{w}}$ with the following properties :

(h1) $\operatorname{Ver}\left(H_{1}\right) \notin T_{w}$,

(h2) $H_{1}$ is tangent to $\bar{X}_{w}$ at its non-singular point, and

(h3) $\operatorname{Sing}\left(\bar{X}_{w}\right) \cap H_{1}=\emptyset$.

The automorphism group $P G L(n+1)$ of $\mathbf{P}^{n}$ acts on $\left(\mathbf{P}^{n}\right)^{-}$and $\mathbf{P}_{*}(\Gamma)$ in such a natural way that $V e r$ is equivariant. Note that $D_{0} \subset \mathbf{P}_{*}(\Gamma)$ is stable under this action. There is an element $g \in P G L(n+1)$ such that $g\left(H_{1}\right)=H_{\infty}$. Con- 
sider the point $g(w) \in D_{0}$, which corresponds to the singular hypersurface $g\left(\bar{X}_{w}\right)$ $\subset \mathbb{P}^{n}$. Then $D_{0}$ is also non-singular at $g(w)$, and the tangent hyperplane $T_{\theta(w)}$ $\subset \mathbb{P}_{*}(\Gamma)$ to $D_{0}$ at $g(w)$ is given by $g\left(T_{w}\right)$. By (h2) and (h3), we see that $g(w)$ $\in D_{0 \infty}$. Because of $(\mathrm{h} 1), \mathfrak{h}_{\infty}=\operatorname{Ver}\left(H_{\infty}\right)=g\left(\operatorname{Ver}\left(H_{1}\right)\right)$ is not contained in $T_{g(w)}$. Hence the line $L_{g(w)}$ connecting $\mathfrak{h}_{\infty}$ and $g(w)$ intersects $D_{0}$ transversely at $g(w)$. Claim 4 is proved.

Now we shall complete the proof of Proposition 9.6.1. The property $(x-1)$ and Proposition 2.3 imply that

$$
\operatorname{Cr}(u)=\bar{c}_{u}^{-1}\left(L_{u}^{o} \cap D_{0}\right) \text { for all } u \in \Delta \text {. }
$$

When $u \in \Delta \backslash\{c\}, L_{u}^{o}$ intersects $D_{0}$ at distinct $N$ points transversely by Proposition 2.4 and (9.6.1). Claim 1 implies that when $w$ moves in $\widetilde{\Delta}$, the points $\tilde{q}_{1}(w)$, $\ldots, \tilde{q}_{N-1}(w)$ are contained in a bounded domain of $\mathbb{A}^{n}$, and hence

$$
\tilde{p}_{1}(w), \ldots, \tilde{p}_{N-1}(w) \text { are contained in a bounded domain of } \mathbb{C} \text {. }
$$

Combining this with Claim 2, we see from (9.6.12) that, when $u$ approaches $c$. one of the intersection points of $L_{u}^{o}$ and $D_{0}$ tends to the point $\mathfrak{h}_{\infty} \in L_{u}$. while the other $N-1$ points remain aloof from $\mathfrak{h}_{\infty}$. Moreover, Claim 4 implies that

$$
\text { these } N-1 \text { points remain distinct even when } u=c \text {. }
$$

These show in particular that the action of $[\gamma]_{*}$ on the set $L_{b}^{o} \cap D_{0}$ of intersection points is trivial. Hence the assertion (1) of Proposition is proved. The assertions (2) and (3) follow from Claim 2 and the fact (9.6.13), respectively. The fact (9.6.14) implies that $p_{1}(c), \ldots, p_{N-1}(c)$ are distinct. The assertion that $p_{i}(c) \neq 0$ for $i=1, \ldots, N-1$ follows because the position of $c$ in $\mathscr{D}_{\infty}$ is general. Indeed, if we replace $c$ with $c^{\prime} \in \mathscr{D}_{\infty}$ such that $f_{c^{\prime}}=f_{c}+a \cdot h^{d}$ for some $a \in \mathbb{C}$, we have $p_{i}\left(c^{\prime}\right)=p_{i}(c)+a$. Thus the assertion (4) is proved.

Remark 9.6.1. The locus $D_{0} \cap D_{\infty}$ consists of two irreducible components; one is the closure of $D_{0 \infty}$ defined above, and the other is the locus of all singular hypersurfaces $\bar{X}$ such that Sing $\bar{X} \cap H_{\infty} \neq \emptyset$.

\section{\$9.7. Proof of Theorem 9.2 .2}

We take an affine line $\mathscr{A} \subset \Gamma$, a s'mall closed disk $\Delta \subset \mathscr{A}$ with the center $c$ $\in \mathscr{A} \cap \mathscr{D}_{\infty}$, and the base point $b \in \partial \Delta$ of the simple loop $\gamma$ around $\mathscr{D}_{\infty}$ as in the beginning of $\S 9.6$. By (9.6.2), we have $K_{b} \subset \mathbb{C}^{\times}$, and the isomorphisms $\Psi_{b}^{0}$ 
and $\Psi_{b}^{\infty}$.

Proposition 9.7.1. There exist a non-zero element $e$ in the kernel of the natural homomorphism $H_{n-1}\left(X_{b}^{0}\right) \rightarrow H_{n-1}\left(\bar{X}_{b}^{0}\right)$ and a $\mathbf{Z}$-linear form $l: H_{n-1}\left(X_{b}^{0}\right) \rightarrow$ $\mathbb{Z}$ such that the monodromy action $[\gamma]_{*}$ on $H_{n-1}\left(X_{b}^{0}\right)$ is given by

$$
x \mapsto x+l(x) \cdot e
$$

Moreover the pair $(e, l)$ is unique up to sign.

This proposition will be proved later together with Proposition 9.7.2 below.

Since $\Delta$ is small enough, Proposition 9.6.1 implies that

$$
\left|p_{N}(\gamma(t))\right|>\left|p_{\imath}(\gamma(t))\right| \text { for } i \neq N
$$

holds for all $t \in I$. Consider the disk

$$
D_{N}^{\infty}:=\left\{z \in \mathbb{C} \cup\{\infty\}:|z| \geq\left|p_{N}(b)\right|\right\}
$$

It is obvious that $K_{b} \cap D_{N}^{\infty}$ is simply-connected and its intersection with $\mathrm{Cr}(b)$ consists of only one point $p_{N}(b)$ because of (9.7.2). By definition, $K_{b} \cap D_{N}^{\infty}$ contains $a_{b}^{\infty}$. Therefore, there exists a unique homotopy class of paths

$$
\left[\xi_{N}^{\infty}\right] \in\left[\mathscr{P}_{b}\left(a_{b}^{\infty}, p_{N}(b)\right)\right]
$$

which is represented by a path $\xi_{N}^{\infty}$ such that

$$
\xi_{N}^{\infty}(I) \subset K_{b} \cap D_{N}^{\infty}
$$

Now by Remarks 9.2.2 and 9.2.3, Theorem 9.2.2 follows from the following:

Proposition 9.7.2. Let

$$
v^{\sim}:=\bar{\theta}\left(\left[\xi_{N}^{\infty}\right]\right)\langle 0\rangle \in H_{n}\left(F_{b}, \partial_{\infty} F_{b}\right)
$$

be the homology class of the lifted thimble $\theta\left(\left[\xi_{N}^{\infty}\right]\right)\langle 0\rangle$, where $\left[\xi_{N}^{\infty}\right] \in\left[\mathscr{P}_{b}\left(a_{b}^{\infty}\right.\right.$, $\left.\left.p_{N}(b)\right)\right]$ is the unique homotopy class of paths characterized by (9.7.3). Then $v$ is primitive, and there is an element $v_{2} \in H_{n}\left(F_{b}\right)$ such that the local monodromy action $[\gamma]_{*}$ on $H_{n}\left(F_{b}, \partial_{0} F_{b}\right)$ along the simple loop $\gamma$ around $\mathscr{D}_{\infty}$ associated with the data $(\mathscr{A}, c, b, \Delta)$ is given $b y$ 


$$
x \mapsto x \pm\left(x, v^{\nu}\right)_{\infty} \cdot\left\{-\Psi_{b}^{0}(e \otimes 1)+v_{2}\right\}
$$

where $e \in H_{n-1}\left(X_{b}^{0}\right)$ is the element in Proposition 9.7.1. Let $\sigma\left[\xi_{N}^{\infty}\right] \in\left[S^{n-1}, X_{b}^{\infty}\right]$ be the vanishing cycle from which the thimble $\theta\left(\left[\xi_{N}^{\infty}\right]\right)$ starts, so that $v^{-}=$ $-\Psi_{b}^{\infty}\left(\bar{\sigma}\left[\xi_{N}^{\infty}\right] \otimes 1\right)$. Then $v_{2}$ is written as follows :

$$
\begin{aligned}
v_{2} & =\Psi_{b}^{\infty}\left(\bar{\sigma}\left[\xi_{N}^{\infty}\right] \otimes\left( \pm q^{-d+1}+a_{-d+2} q^{-d+2}+\cdots+a_{-1} q^{-1}\right)\right)+\Psi_{b}^{\infty}(w \otimes 1) \\
& =-\left( \pm q^{-d+1}+a_{-d+2} q^{-d+2}+\cdots+a_{-1} q^{-1}\right) \cdot v^{\nu}+\Psi_{b}^{\infty}(w \otimes 1)
\end{aligned}
$$

where $a_{-d+2, \ldots, a_{-1}}$ are certain integers, and $w \in H_{n-1}\left(X_{b}^{\infty}\right)$ is a certain homology class.

Remark 9.7.1. The fact that the coefficient of $q^{-d+1}$ in (9.7.5) is 1 or -1 plays an important role in the proof of Irreducibility Theorem in the next section.

Remark 9.7.2. We can determine neither the combination of signs in (9.7.4) and $(9.7 .5)$, nor the values of the integers $a_{-d+2} \ldots, a_{-1}$. We would like to fill up this unsatisfactory part of the theory in the future.

Proofs of Propositions 9.7.1 and 9.7.2. We write the set $\mathrm{Cr}(b)$ simply by $\left\{p_{1}, \ldots, p_{N}\right\}$ instead of $\left\{p_{1}(b), \ldots, p_{N}(b)\right\}$. The movement $M_{r}$ of the distinguished points $\mathscr{S}_{b}:=\mathscr{C}_{r}(b) \cup Z_{b}^{0} \cup Z_{b}^{\infty}$ in $\mathbb{C}$ along the loop $\gamma$ is homotopically equivalent to the movement $\mathcal{M}_{r}^{\prime}: I \times \mathscr{\Theta}_{b} \rightarrow \mathbb{C}$ described as follows: the points $a_{b}^{0}\langle\nu\rangle$ and $a_{b}^{\infty}\langle\nu\rangle$ remain fixed, the points $p_{i}\langle\nu\rangle$ also remain fixed if $i \neq N$, and they stay left-hand side of the vertical line

$$
\Lambda_{\infty}:=\log \left|p_{N}\right|+\sqrt{-1} \mathbb{R}=e^{-1}\left(\partial D_{N}^{\infty}\right),
$$

while the point $p_{N}\langle\nu\rangle$ moves down to $p_{N}\langle\nu-d+1\rangle$ along the vertical line $\Lambda_{\infty}$. This can be seen as follows. Let $p_{i}(\Delta) \subset \mathbb{C} U\{\infty\}$ be the image of the meromorphic function $\Delta \rightarrow \mathbb{C} \cup\{\infty\}$ corresponding to the $i$-th critical value, whose existence has been proved in Proposition 9.6.1. Since $\Delta$ is small enough, Proposition 9.6.1 implies that

$$
p_{i}(\Delta) \subset \mathbb{C}^{\times} \quad \text { if } \quad i \neq N,
$$

and

$$
p_{i}(\Delta) \cap p_{j}(\Delta)=\emptyset \quad \text { if } \quad i \neq j .
$$


The movements of $a_{b}^{0}\langle\nu\rangle$ and $a_{b}^{\infty}\langle\nu\rangle$ are homotopically equivalent to the non-movement by Reduction 1 or Remark 9.3.1 in §9.3. By (9.7.6) and (9.7.7), if $i \neq N$, then the movement of $p_{i}\langle\nu\rangle$ is also homotopically equivalent to the non-movement by Reduction 2 in $\$ 9.3$. On the other hand, Proposition 9.6.1 (2) implies that $p_{N} \in \mathbb{C}^{\times}$makes round trips along a large circle in the clockwise direction $(d-1)$-times. Combining this with (9.7.7), the trace of the movement of $p_{N}\langle\nu\rangle$ can be deformed to the segment of $\Lambda_{\infty}$ between $p_{N}\langle\nu\rangle$ and $p_{N}\langle\nu-d+1\rangle$ without affecting the movements of the other distinguished points.

By (9.7.2), there exists a $K$-regular system $\left\{\xi_{1}^{0}, \ldots, \xi_{N}^{0}\right\}$ of paths from $a_{b}^{0}$ which satisfies the following property:

$$
\xi_{\imath}^{0}(I) \cap D_{N}^{\infty}=\left\{\begin{array}{lll}
\emptyset & \text { if } & i \neq N, \text { and } \\
\left\{p_{N}\right\} & \text { if } & i=N .
\end{array}\right.
$$

We choose a vanishing cycle

$$
\sigma_{i}^{0}:=\sigma\left[\xi_{l}^{0}\right] \in\left[S^{n-1}, X_{b}^{0}\right]
$$

for each $\left[\xi_{i}^{0}\right]$. By Proposition 5.1, their homology classes $\bar{\sigma}_{1}^{0}, \ldots, \bar{\sigma}_{N}^{0}$ form a set of basis of the free $\mathbb{Z}$-module $H_{n-1}\left(X_{b}^{0}\right)$. We define a $\mathbb{Z}$-linear form $l: H_{n-1}\left(X_{b}^{0}\right)$ $\rightarrow \mathbb{Z}$ by

$$
l\left(\bar{\sigma}_{i}^{0}\right):= \begin{cases}0 & \text { if } i \neq N, \text { and } \\ 1 & \text { if } i=N .\end{cases}
$$

By Proposition 9.6.1(1) and (9.6.1). [ $\gamma]_{*}$ acts on the set $\left[\mathscr{P}_{b}\left(a_{b}^{0}, p_{\imath}\right)\right]$, and if $i \neq$ $N$, this action lifts to the action on $\left[\mathscr{P}_{b}^{\sim}\left(a_{b}^{0}\langle\nu\rangle, p_{i}\langle\nu\rangle\right)\right]$ because (9.7.6) implies $[\gamma]_{*}\left(p_{i}\langle\nu\rangle\right)=p_{i}\langle\nu\rangle$ for $i \neq N$. Since $\xi_{i}^{0}(I) \subset K_{b}$, we have $\left[\xi_{i}^{0}\langle\nu\rangle\right] \in$ $\left[\mathscr{P}_{b} \tilde{b}\left(a_{b}^{0}\langle\nu\rangle, p_{l}\langle\nu\rangle\right)\right]$. From (9.7.6) and (9.7.7), we can easily see that

$$
[\gamma]_{*}\left(\left[\xi_{i}^{0}\right]\right)=\left[\xi_{i}^{0}\right] \text { in }\left[\mathscr{P}_{b}\left(a_{b}^{0}, p_{i}\right)\right] \text { for } i \neq N \text {, }
$$

and, combining this with $(9.3 .8)$, we have

$$
[\gamma]_{*}\left(\left[\xi_{i}^{0}\langle\nu\rangle\right]\right)=\left[\xi_{i}^{0}\langle\nu\rangle\right] \text { in }\left[\mathscr{P}_{b}^{\sim}\left(a_{b}^{0}\langle\nu\rangle, p_{i}\langle\nu\rangle\right)\right] \text { for } i \neq N \text {. }
$$

This also can be seen as follows. Because the path $\xi_{i}^{0}\langle\nu\rangle$ is disjoint from $\Lambda_{\infty}$ for $i \neq N$ by (9.7.8), the description of the movement $\mathcal{M}_{r}^{\prime}$ above implies that, if $i$ $\neq N$, then $\xi_{i}^{0}\langle\nu\rangle$ is not affected by the movement of $p_{N}\langle\mu\rangle$ for any $\mu \in \mathbb{Z}$. 
Since $[\gamma]_{*}\left(\sigma_{i}^{0}\right) \in\left[S^{n-1}, X_{b}^{0}\right]$ is a vanishing cycle for $[\gamma]_{*}\left(\left[\xi_{i}^{0}\right]\right),(9.7 .10)$ implies that, if $i \neq N$, then $[\gamma]_{*}\left(\sigma_{i}^{0}\right)$ is either $\sigma_{i}^{0}$ or $-\sigma_{i}^{0}$. We shall show that

$$
[\gamma]_{*}\left(\sigma_{i}^{0}\right)=\sigma_{i}^{0} \text { for } i \neq N \text {, }
$$

and that

$$
e:=[\gamma]_{*}\left(\bar{\sigma}_{N}^{0}\right)-\bar{\sigma}_{N}^{0} \in \operatorname{Ker}\left(H_{n-1}\left(X_{b}^{0}\right) \rightarrow H_{n-1}\left(\bar{X}_{b}^{0}\right)\right)
$$

First note that the action of $[\gamma]_{*}$ on $H_{n-1}\left(\bar{X}_{b}^{0}\right)$ is trivial. Indeed, the property $(\mathrm{x}-1)$ in $\$ 9.6$ implies that $[\gamma]_{*}$ acts on $H_{n-1}\left(\bar{X}_{b}\right)$ trivially. By the same argument as Lemma 5.1, $[\gamma] *$ acts on $H_{n-1}\left(\bar{X}_{b}^{0}\right)$ also trivially. This, in particular, implies (9.7.13). Second, note that the image $\left(\bar{\sigma}_{i}^{0}\right)^{\prime}$ of $\bar{\sigma}_{i}^{0}$ by the natural homomorphism $H_{n-1}\left(X_{b}^{0}\right) \rightarrow H_{n-1}\left(\bar{X}_{b}^{0}\right)$ is non-zero for $i=1, \ldots, N$. Indeed, the image of $H_{n-1}\left(X_{b}^{0}\right) \rightarrow H_{n-1}\left(\bar{X}_{b}^{0}\right)$ is, by definition, $H_{\text {prim }}^{n-1}\left(\bar{X}_{b}^{0}\right)$ in the exact sequence (5.11). Theorem L3 in $\S 5$ tells us that, for each $i$, the element $\left(\bar{\sigma}_{i}^{0}\right)^{\prime}$ generates $H_{\text {prim }}^{n-1}\left(\bar{X}_{b}^{0}\right) \otimes \mathbb{Q}$ as a $\mathbb{Q}\left[\pi_{1}(U)\right]$-module. Therefore, $\left(\bar{\sigma}_{i}^{0}\right)^{\prime}$ is not zero for each $i=1, \ldots, N$. Combining these two facts, we see that $[\gamma]_{*}\left(\bar{\sigma}_{1}^{0}\right)$ cannot be $-\bar{\sigma}_{i}^{0}$. Therefore, $[\gamma]_{*}\left(\sigma_{i}^{0}\right)$ cannot be $-\sigma_{i}^{0}$. Hence (9.7.12) is proved. We shall show that $e$ defined in (9.7.13) is non-zero. If it were zero, then $[\gamma]_{*}$ would act on $H_{n-1}\left(X_{b}^{0}\right)$ trivially because of (9.7.12). However, since $Y_{c}=\bar{X}_{c}$ $\cap H_{\infty}=\bar{X}_{c}^{0} \cap H_{\infty}$ has an ordinary double point by the property $(\mathrm{x}-3)$ in $\S 9.6$, the action of $[\gamma]_{*}$ on the non-zero sub-module $H_{\mathrm{prim}}^{n-2}\left(Y_{b}\right)$ of $H_{n-1}\left(X_{b}^{0}\right)$ is non-trivial, because $H_{\text {prim }}^{n-2}\left(Y_{b}\right) \otimes \mathbb{Q}$ corresponds to the module of "vanishing cycles" in $H_{n-2}\left(Y_{b}\right) \otimes \mathbb{Q}$ in the sense of $[8 ; \S 3]$ by the Poincaré duality.

Then, by (9.7.12) and the definitions (9.7.9) and (9.7.13), we obtain

$$
[\gamma]_{*}(x)=x+l(x) \cdot e \text { for all } x \in H_{n-1}\left(X_{b}^{0}\right)
$$

This formula being established, $e$ is characterized as a generater of the image of the endomorphism Id $-[\gamma]_{*}$ on $H_{n-1}\left(X_{b}^{0}\right)$, which is a free $\mathbb{Z}$-module of rank 1 , and hence $e$ is uniquely determined up to sign. Therefore the pair $(e, l)$ is also unique up to sign. Thus Proposition 9.7.1 is proved.

Let

$$
\theta_{i}^{0}:=\theta\left(\left[\xi_{i}^{0}\right], \sigma_{l}^{0}\right) \in\left[\left(C S^{n-1}, S^{n-1}\right),\left(E_{b}, X_{b}^{0}\right)\right]
$$


denote the thimble for $\left[\xi_{i}^{0}\right]$ starting from $\sigma_{i}^{0}$, and let

$$
\theta_{i}^{0}\langle\nu\rangle \in\left[\left(C S^{n-1}, S^{n-1}\right),\left(F_{b}, X_{b}^{0}\langle\nu\rangle\right)\right]
$$

denote its lifting, which is the thimble for $\left[\xi_{\imath}^{0}\langle\nu\rangle\right] \in\left[\mathscr{P}_{b}^{\sim}\left(a_{b}^{0}\langle\nu\rangle, p_{\imath}\langle\nu\rangle\right)\right]$ starting from $\sigma_{i}^{0}\langle\nu\rangle$. By Proposition 7.3, the homology classes $\bar{\theta}_{1}^{0}\langle 0\rangle, \ldots, \bar{\theta}_{N}^{0}\langle 0\rangle$ in $H_{n}\left(F_{b}, \partial_{0} F_{b}\right)$ form a set of basis over $\mathbb{Z}\left[q, q^{-1}\right]$. Hence it is enough to prove (9.7.4) when $x$ runs through the set of these classes. By (9.7.3) and (9.7.8), the paths $\xi_{\imath}^{0}$ and $\xi_{N}^{\infty}$ are disjoint if $i \neq N$, and the paths $\xi_{N}^{0}$ and $\xi_{N}^{\infty}$ have a common ending point $p_{N}$ as their only intersection. Hence, by Lemma 8.2, we have

$$
\left(\bar{\theta}_{i}^{0}\langle 0\rangle, v^{\nu}\right)_{\infty}=\left(\bar{\theta}\left(\left[\xi_{i}^{0}\right]\right)\langle 0\rangle, \bar{\theta}\left(\left[\xi_{N}^{\infty}\right]\right)\langle 0\rangle\right)_{\infty}= \begin{cases}0 & \text { if } i \neq N \text {, and } \\ \pm 1 & \text { if } i=N .\end{cases}
$$

In particular, this shows that $v^{\sim}$ is primitive.

We can and will choose the sign of $\sigma_{N}^{0}$ in such a way that

$$
\left(\bar{\theta}_{N}^{0}\langle 0\rangle, v^{\sim}\right)_{\infty}=1
$$

From the formulae (9.3.4) and (9.3.6), the results (9.7.11) and (9.7.12) imply that

$$
[\gamma]_{*}\left(\theta_{i}^{0}\langle 0\rangle\right)=\theta_{i}^{0}\langle 0\rangle \text { for } i \neq N
$$

Combining this with (9.7.14) and (9.7.15), we see that the action $[\gamma]_{*}$ on $H_{n}\left(F_{b}\right.$, $\left.\partial_{0} F_{b}\right)$ is given by

$$
x \mapsto x+\left(x, v^{2}\right)_{\infty} \cdot v^{\prime}
$$

where

$$
v^{\prime}:=[\gamma]_{*}\left(\bar{\theta}_{N}^{0}\langle 0\rangle\right)-\bar{\theta}_{N}^{0}\langle 0\rangle
$$

Now we shall show that this homology class $v^{\prime}$ is equal, up to sign, with $-\Psi_{b}^{0}(e \otimes 1)+v_{2}$. where $v_{2}$ is an element of $H_{n}\left(F_{b}\right)$ which can be written in the form (9.7.5).

From the description of the movement $\mathcal{M}_{r}^{\prime}$, we see that $[\gamma]_{*}\left(\left[\xi_{N}^{0}\right]\langle\nu\rangle\right)$, which is an element of $\left[\mathscr{P}_{b}^{\sim}\left(a_{b}^{0}\langle\nu\rangle, p_{N}\langle\nu-d+1\rangle\right)\right]$, is represented by a path 
$\xi^{\prime \prime}\langle\nu\rangle$ as follows. Note that $\xi_{N}^{0}\langle\nu\rangle(I)$ is on the left-hand side of the vertical line $\Lambda_{\infty}=\log \left|p_{N}\right|+\sqrt{-1} \mathbb{R}$ because of (9.7.8). Then the path $\xi^{\prime \prime}\langle\nu\rangle$ starts from $a_{b}^{0}\langle\nu\rangle$, and goes to a point $p_{N}^{\prime}\langle\nu\rangle:=p_{N}\langle\nu\rangle-\kappa^{\prime}$ along $\xi_{N}^{0}\langle\nu\rangle$ where $\kappa^{\prime}$ is a sufficiently small complex number with $\operatorname{Re} \kappa^{\prime}>0$, goes down to $p_{N}^{\prime}\langle\nu-d+$ $1\rangle=p_{N}\langle\nu-d+1\rangle-\kappa^{\prime}$ along the vertical line parallel to $\Lambda_{\infty}$, and then reaches $p_{N}\langle\nu-d+1\rangle$ along $\xi_{N}^{0}\langle\nu-d+1\rangle$.

We define the path $\xi_{N}^{0 \infty}\langle\nu\rangle$ from $a_{b}^{0}\langle\nu\rangle$ to $a_{b}^{\infty}\langle\nu\rangle$ as follows. Note that $\xi_{N}^{\infty}\langle\nu\rangle(I)$ is on the right-hand side of the vertical line $\Lambda_{\infty}$ because of (9.7.3). Then $\xi_{N}^{0 \infty}\langle\nu\rangle$ goes from $a_{b}^{0}\langle\nu\rangle$ to the point $p_{N}^{\prime}\langle\nu\rangle$ along $\xi_{N}^{0}\langle\nu\rangle$, and draws an arc on the circle of radius $\left|\kappa^{\prime}\right|$ with the center $p_{N}\langle\nu\rangle$ in the counter-clockwise direction to a point $p_{N}^{\prime \prime}\langle\nu\rangle:=p_{N}\langle\nu\rangle+\kappa^{\prime \prime}$ on $\xi_{N}^{\infty}\langle\nu\rangle(I)$, where $\kappa^{\prime \prime}$ is a complex number such that $\left|\kappa^{\prime}\right|=\left|\kappa^{\prime \prime}\right|$ and $\left.\operatorname{Re} \kappa^{\prime \prime}\right\rangle 0$, and then goes to $a_{b}^{\infty}\langle\nu\rangle$ along $\xi_{N}^{\infty}\langle\nu\rangle^{-1}$. Note that $\xi_{N}^{0 \infty}\langle\nu\rangle$ is a path in $K_{b}\langle\nu\rangle$.

We put

$$
p_{N}^{\prime \prime}:=e\left(p_{N}^{\prime \prime}\langle\nu\rangle\right) \in \xi_{N}^{\infty}(I) \subset \mathbb{C}^{\times},
$$

and define a loop $\tau$ with the base point $a_{b}^{\infty}$ in $\mathbb{C}^{\times} \backslash \mathrm{Cr}(b)$ as follows : $\tau$ goes from $a_{b}^{\infty}$ to $p_{N}^{\prime \prime}$ along $\xi_{N}^{\infty}$, draws a circle of radius $\left|p_{N}-p_{N}^{\prime \prime}\right|$ with the center $p_{N}$ in the counter-clockwise direction, and then goes back to $a_{b}^{\infty}$ along $\left(\xi_{N}^{\infty}\right)^{-1}$. Note that $\tau$ is a path in $K_{b}$.

Now we are going to be interested exclusively in the case $\nu=0$. It is easy to see that

$$
[\gamma]_{*}\left(\left[\xi_{N}^{0}\langle 0\rangle\right]\right)=\left[\xi^{\prime \prime}\langle 0\rangle\right]=[\zeta] \text { in }\left[\mathscr{P}_{b}^{\sim}\left(a_{b}^{0}\langle 0\rangle, p_{N}\langle-d+1\rangle\right)\right] \text {, }
$$

where $\zeta:=\zeta^{\prime} \cdot \xi_{N}^{0 \infty}\langle 0\rangle$ and

$$
\begin{aligned}
\zeta^{\prime}:= & \xi_{N}^{\infty}\langle-d+1\rangle \cdot\left(\delta_{b}^{\infty}\langle-d+1\rangle\right)^{-1} \cdot \tau\langle-d+2\rangle \cdot\left(\delta_{b}^{\infty}\langle-d+2\rangle\right)^{-1} \ldots \\
& \cdots \tau\langle-3\rangle \cdot\left(\delta_{b}^{\infty}\langle-3\rangle\right)^{-1} \cdot \tau\langle-2\rangle \cdot\left(\delta_{b}^{\infty}\langle-2\rangle\right)^{-1} \cdot \tau\langle-1\rangle \cdot\left(\delta_{b}^{\infty}\langle-1\rangle\right)^{-1} .
\end{aligned}
$$

(See $\S 6$ for the definition of the path $\delta_{b}^{\infty}$.) We put

$$
\sigma^{*}:=[\gamma]_{*}\left(\sigma_{N}^{0}\langle 0\rangle\right)
$$

This is a vanishing cycle for $[\zeta]$ in $X_{b}^{0}\langle 0\rangle$, and by formula (9.3.4), we have 


$$
[\gamma]_{*}\left(\theta_{N}^{0}\langle 0\rangle\right)=\theta\left([\zeta], \sigma^{*}\right) \in\left[\left(C S^{n-1}, S^{n-1}\right),\left(F_{b}, X_{b}^{0}\langle 0\rangle\right)\right]
$$

In order to determine its homology class, we choose a continuous map $T: C S^{n-1}$ $\rightarrow F_{b}$ which represents $\theta\left([\zeta], \sigma^{*}\right)$ over the path $\zeta$. Let $T_{0}$ and $T_{1}$ denote the restrictions of $T$ to the sub-paths $\xi_{N}^{0 \infty}\langle 0\rangle$ and $\zeta^{\prime}$ of $\zeta$, respectively. As $n$-chains in $F_{b}$, we have $T=T_{0}+T_{1}$. Then $T_{0}$ is a continuous map from $I \times$ $S^{n-1}$ to $\phi_{b}^{-1}\left(K_{b}\langle 0\rangle\right)$ because of $\xi_{N}^{0 \infty}\langle 0\rangle(I) \subset K_{b}\langle 0\rangle$, and $T_{1}: C S^{n-1} \rightarrow F_{b}$ represents a thimble for $\left[\zeta^{\prime}\right]$ over $\zeta^{\prime}$. Their boundaries are given by

$$
\partial T_{0}=-S^{*}+S^{\prime}, \text { and } \partial T_{1}=-S^{\prime}
$$

where $S^{*}: S^{n-1} \rightarrow X_{b}^{0}\langle 0\rangle$ represents the vanishing cycle $\sigma^{*}=[\gamma]_{*}\left(\sigma_{N}^{0}\langle 0\rangle\right)$, and $S^{\prime}: S^{n-1} \rightarrow X_{b}^{\infty}\langle 0\rangle$ represents a vanishing cycle for $\left[\zeta^{\prime}\right]$. Since $\phi_{b}^{-1}\left(K_{b}\right.$

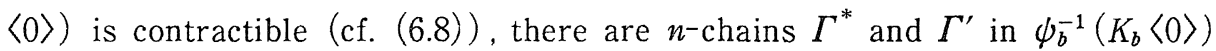
such that $\partial \Gamma^{*}=S^{*}$ and $\partial \Gamma^{\prime}=S^{\prime}$. The sum $T_{0}+\Gamma^{*}-\Gamma^{\prime}$ is an $n^{-c y c l e}$ in $\phi_{b}^{-1}$ $\left(K_{b}\langle 0\rangle\right)$, which is obviously homologous to zero because of the contractibility of $\phi_{b}^{-1}\left(K_{b}\langle 0\rangle\right)$. Hence we have

$$
[\gamma]_{*}\left(\bar{\theta}_{N}^{0}\langle 0\rangle\right)=[T]=\left[-\Gamma^{*}\right]+\left[\Gamma^{\prime}+T_{1}\right] \text { in } H_{n}\left(F_{b}, \partial_{0} F_{b}\right)
$$

Note that $\Gamma^{\prime}+T_{1}$ is an $n$-cycle in $F_{b}$, because $\partial T_{1}=-S^{\prime}$. We put

$$
v_{2}:=\left[\Gamma^{\prime}+T_{1}\right] \in H_{n}\left(F_{b}\right) .
$$

Since the homology class of the boundary $\partial \Gamma^{*}=S^{*}$ in $X_{b}^{0}\langle 0\rangle$ is $\bar{\sigma}^{*}=$ $[\gamma]_{*}\left(\bar{\sigma}_{N}^{0}\langle 0\rangle\right)$, it is mapped to $[\gamma]_{*}\left(\bar{\sigma}_{N}^{0}\right)$ by the isomorphism $H_{n-1}\left(X_{b}^{0}\langle 0\rangle\right) \cong$ $H_{n-1}\left(X_{b}^{0}\right)$ induced from (6.1). By the definition of $e$ (cf. (9.7.13)), we have $[\gamma]_{*}\left(\bar{\sigma}_{N}^{0}\right)=\bar{\sigma}_{N}^{0}+e$. Since $\Gamma^{*}$ is contained in $\phi_{b}^{-1}\left(K_{b}\langle 0\rangle\right)$, we see from Corollary 6.2 that

$$
-\left[\Gamma^{*}\right]=-\Psi_{b}^{0}\left([\gamma]_{*}\left(\bar{\sigma}_{N}^{0}\right) \otimes 1\right)=-\Psi_{b}^{0}\left(\bar{\sigma}_{N}^{0} \otimes 1\right)-\Psi_{b}^{0}(e \otimes 1) .
$$

On the other hand, we have $\bar{\theta}_{N}^{0}\langle 0\rangle=-\Psi_{b}^{0}\left(\bar{\sigma}_{N}^{0} \otimes 1\right)$ because of Proposition 7.2. Combining this with $(9.7 .16)-(9.7 .19)$, we obtain

$$
v^{\prime}=[\gamma]_{*}\left(\bar{\theta}_{N}^{0}\langle 0\rangle\right)-\bar{\theta}_{N}^{0}\langle 0\rangle=-\Psi_{b}^{0}(e \otimes 1)+\left[\Gamma^{\prime}+T_{1}\right]=-\Psi_{b}^{0}(e \otimes 1)+v_{2}
$$

We shall express $v_{2}=\left[\Gamma^{\prime}+T_{1}\right] \in H_{n}\left(F_{b}\right)$ in terms of $\Psi_{b}^{\infty}$, and show that the expression is of the form (9.7.5). For $\mu=-1,-2, \ldots,-d+2$, let $T_{(\mu)}$ de- 
note the restriction of $T_{1}$ to the sub-path $\tau\langle\mu\rangle$ of $\zeta^{\prime}$, and let $T_{(-d+1)}$ denote the restriction of $T_{1}$ to the ending piece $\xi_{N}^{\infty}\langle-d+1\rangle$ of $\zeta^{\prime}$. Since the restriction of $T_{1}$ to $\left(\delta_{b}^{\infty}\langle\nu\rangle\right)^{-1}$ is contained in $\partial_{\infty} F_{b}$ for all $\nu$, we have

$$
v_{2}=\left[\Gamma^{\prime}+T_{1}\right]=\left[\Gamma^{\prime}\right]+\left[T_{(-1)}\right]+\left[T_{(-2)}\right]+\cdots+\left[T_{(-d+1)}\right] \text { in } H_{n}\left(F_{b}, \partial_{\infty} F_{b}\right) .
$$

We define $w \in H_{n-1}\left(X_{b}^{\infty}\right)$ to be the image of the homology class

$$
\left[\partial \Gamma^{\prime}\right]=\left[S^{\prime}\right] \in H_{n-1}\left(X_{b}^{\infty}\langle 0\rangle\right)
$$

by the isomorphism $H_{n-1}\left(X_{b}^{\infty}\langle 0\rangle\right) \cong H_{n-1}\left(X_{b}^{\infty}\right)$ induced from (6.1). Since $\Gamma^{\prime}$ is contained in $\phi_{b}^{-1}\left(K_{b}\langle 0\rangle\right)$, we see from Corollary 6.2 that

$$
\left[\Gamma^{\prime}\right]=\Psi_{b}^{\infty}(w \otimes 1)
$$

The continuous map $T_{(-d+1)}: C S^{n-1} \rightarrow \phi_{b}^{-1}\left(K_{b}\langle-d+1\rangle\right)$ represents a thimble for $\left[\xi_{N}^{\infty}\langle-d+1\rangle\right]$ over the path $\xi_{N}^{\infty}\langle-d+1\rangle$, which is either $\theta\left(\left[\xi_{N}^{\infty}\right]\right)\langle-d+1\rangle$ or $-\theta\left(\left[\xi_{N}^{\infty}\right]\right)\langle-d+1\rangle$. Therefore we have

$$
\left[T_{(-d+1)}\right]= \pm \bar{\theta}\left(\left[\xi_{N}^{\infty}\right]\right)\langle-d+1\rangle= \pm q^{-d+1} \bar{\theta}\left(\left[\xi_{N}^{\infty}\right]\right)\langle 0\rangle= \pm q^{-d+1} v^{\circ}
$$

For $\mu=-1, \ldots,-d+2$, the boundary of the $n$-chain $T_{(\mu)}: I \times S^{n-1} \rightarrow \phi_{b}^{-1}\left(K_{b}\langle\mu\rangle\right)$ is of the form $-S_{\mu}+S_{\mu}^{\prime}$, where $S_{\mu}$ and $S_{\mu}^{\prime}$ are continuous maps from $S^{n-1}$ to $X_{b}^{\infty}\langle\mu\rangle$. Their homology classes are related by

$$
\left[S_{\mu}^{\prime}\right]=[\tau\langle\mu\rangle]_{*}\left(\left[S_{\mu}\right]\right) \text { in } H_{n-1}\left(X_{b}^{\infty}\langle\mu\rangle\right)
$$

By Theorem L1(2) in $\S 4$, the difference

$$
\partial\left[T_{(\mu)}\right]=\left[S_{\mu}^{\prime}\right]-\left[S_{\mu}\right]=\left([\tau\langle\mu\rangle]_{*}-1\right)\left[S_{\mu}\right]
$$

is a multiple of the homology class of a vanishing cycle in $X_{b}^{\infty}\langle\mu\rangle$ for $\left[\xi_{b}^{\infty}\langle\mu\rangle\right]$; that is, it is written as $a_{\mu} \bar{\sigma}\left[\xi_{N}^{\infty}\right]\langle\mu\rangle$ by some integer $a_{\mu}$. The class $\bar{\sigma}\left[\xi_{N}^{\infty}\right]\langle\mu\rangle$ is mapped to $\bar{\sigma}\left[\xi_{N}^{\infty}\right]$ by the isomorphism $H_{n-1}\left(X_{b}^{\infty}\langle\mu\rangle\right) \cong H_{n-1}\left(X_{b}^{\infty}\right)$ induced from (6.1). Since $\tau\langle\mu\rangle(I) \subset K_{b}\langle\mu\rangle, T_{(\mu)}$ is contained in $\phi_{b}^{-1}\left(K_{b}\langle\mu\rangle\right)$. Therefore, we see from Corollary 6.2 that

$(9.7 .23)\left[T_{(\mu)}\right]=a_{\mu} \cdot \Psi_{b}^{\infty}\left(\bar{\sigma}\left[\xi_{N}^{\infty}\right] \otimes q^{\mu}\right)=-a_{\mu} \cdot q^{\mu} \cdot \bar{\theta}\left(\left[\xi_{N}^{\infty}\right]\right)\langle 0\rangle=-a_{\mu} \cdot q^{\mu} \cdot v^{\nu}$ 
Combining (9.7.20)-(9.7.23), we get

$$
v_{2}=\Psi_{b}^{\infty}(w \otimes 1)-\left( \pm q^{-d+1}+a_{-d+2} q^{-d+2}+\cdots+a_{-2} q^{-2}+a_{-1} q^{-1}\right) \cdot v^{\llcorner}
$$

and hence we get (9.7.5).

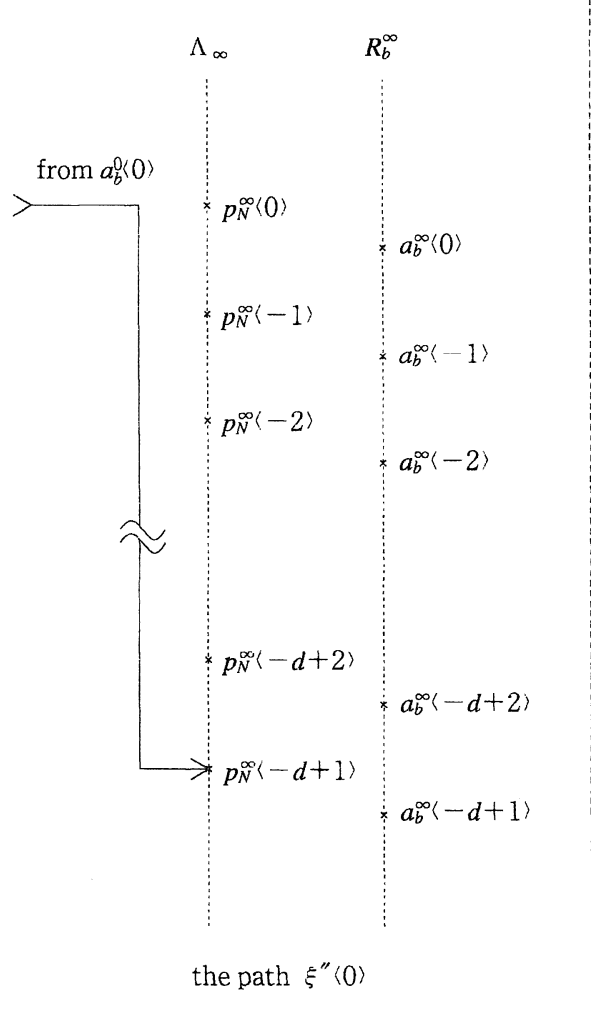

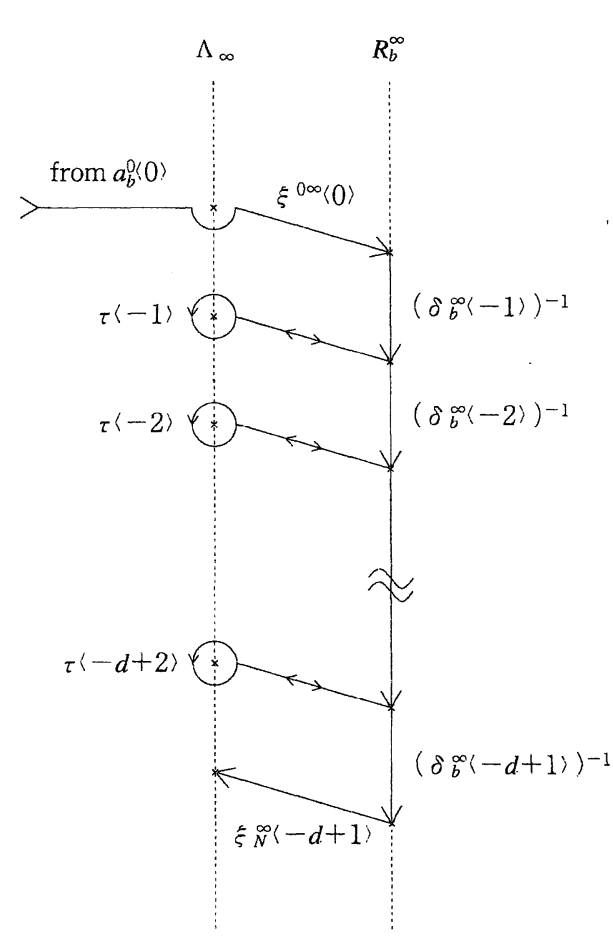

the path $\zeta$

Figure 11

Again, by Remarks 9.2.2 and 9.2.3, we get the following:

Corollary 9.7.1. Let $\gamma$ and $\gamma^{\prime}$ be simple loops around $\mathscr{D}_{\infty}$ with the base point $b$ and $b^{\prime}$, respectively. Let $\alpha$ be a path from $b$ to $b^{\prime}$ in $U$ such that $[\alpha]^{-1}\left[\gamma^{\prime}\right][\alpha]$ $=[\gamma]$ holds in $\pi_{1}(\mathcal{U}, b)$. Then we have a congruence $\left(v\left[\gamma^{\prime}\right], v^{\vee}\left[\gamma^{\prime}\right]\right) \equiv[\alpha]_{*}(v[\gamma]$, $\left.v^{\sim}[\gamma]\right)$ modulo $U\left(\mathbb{Z}\left[q, q^{-1}\right]\right)$ in $H_{n}\left(F_{b^{\prime}}, \partial_{0} F_{b^{\prime}}\right) \times H_{n}\left(F_{b^{\prime}}, \partial_{\infty} F_{b^{\prime}}\right)$. 


\section{\$10. Irreducibility of the Monodromy Representation}

Let $b$ be a base point of $\mathcal{U}$. In this section, we deal with the vector space

$$
H_{n}\left(F_{b}\right) \otimes_{\mathbb{Z}\left[q, q^{-1}\right]} \mathbb{Q}(q)
$$

over the quotient field $\mathbb{Q}(q)$ of $\mathbb{Z}\left[q, q^{-1}\right]$. For brevity, we denote this space by $H_{n}\left(F_{b}\right) \otimes \mathbb{Q}(q)$. Let $\overline{\mathbb{Q}(q)}$ be the algebraic closure of $\mathbb{Q}(q)$. A representation on $H_{n}\left(F_{b}\right) \otimes \mathbb{Q}(q)$ is said to be absolutely irreducible if the induced representation on $H_{n}\left(F_{b}\right) \otimes \bar{Q}(q)$ is also irreducible. The purpose of this section is to prove the following:

Ilreeducibillity Theorem. The monodromy representation of $\pi_{1}(U, b)$ on $H_{n}\left(F_{b}\right) \otimes \mathbb{Q}(q)$ is absolutely irreducible.

Proof. First remark that the natural isomorphisms in Corollary 6.4 enable us to apply Theorems 9.2 .1 and 9.2 .2 to the representation of $\pi_{1}(\mathcal{U}, b)$ on $H_{n}\left(F_{b}\right) \otimes \mathbb{Q}(q)$. In particular, we can consider the homology class $v[\gamma]$ and its dual $v^{\sim}[\gamma]$ as elements of $H_{n}\left(F_{b}\right) \otimes \mathbb{Q}(q)$ for any simple loop $\gamma$ around $\mathscr{D}_{0}$ or $\mathscr{D}_{\infty}$.

Let $x$ be an arbitrary non-zero element of $H_{n}\left(F_{b}\right) \otimes \overline{\mathbb{Q}(q)}$, and let $M$ be the smallest $\overline{\mathbb{Q}(q)}\left[\pi_{1}(U, b)\right]$-submodule of $H_{n}\left(F_{b}\right) \otimes \overline{\mathbb{Q}(q)}$ containing $x$. We have to show that $M$ coincides with the total space. For this purpose, it suffices to prove that $M$ contains an element $v[\gamma] \in H_{n}\left(F_{b}\right)$, where $\gamma$ is a simple loop around $\mathscr{D}_{0}$, because of Theorem 9.5.1.

We consider the vector space $\Gamma=\Gamma\left(\mathbb{P}^{n}, \mathscr{O}(d)\right)$ as an affine part of a projective space $\mathbb{P}^{\operatorname{dim} \Gamma}$, and let $\mathscr{H}$ be the hyperplane $\mathbb{P}^{\operatorname{dim} \Gamma \backslash \Gamma}$. Then $\mathcal{U}$ is the complement to the reducible projective hypersurface $\overline{\mathscr{D}}_{0} \cup \overline{\mathscr{D}}_{\infty} \cup \mathscr{H}$, where $\overline{\mathscr{D}}_{0}$ and $\overline{\mathscr{D}}_{\infty}$ denote the closures of $\mathscr{D}_{0}$ and $\mathscr{D}_{\infty}$, respectively. Hence, Zariski's hyperplane section theorem $([14],[7])$ implies that $\pi_{1}(U, b)$ is generated by the homotopy classes of simple loops around $\mathscr{D}_{0}$ and $\mathscr{D}_{\infty}$. In particular, the generater $\mathfrak{c} \in$ $\pi_{1}(U, b)$ of the kernel of the natural homomorphism $\pi_{1}(U) \rightarrow \pi_{1}(U)$ is written as a product

$$
\left[\gamma_{1}\right]^{\delta_{1}} \cdot\left[\gamma_{2}\right]^{\delta_{2}} \cdot \cdots \cdot\left[\gamma_{k}\right]^{\delta_{k}}
$$

where each $\gamma_{i}$ is a simple loop around $\mathscr{D}_{0}$ or $\mathscr{D}_{\infty}$, and $\delta_{i}$ is \pm 1 . By Proposition 1.1 , we have 


$$
\mathfrak{c}_{*}(x)=q x \neq x
$$

Hence there exists at least one element among $\left[\gamma_{1}\right], \ldots,\left[\gamma_{k}\right]$, say $\left[\gamma_{l}\right]$, such that $\left[\gamma_{l}\right]_{*}(x) \neq x$. By Theorems 9.2.1 and 9.2.2, we have

$$
\left[\gamma_{l}\right]_{*}(x)-x=a \cdot v\left[\gamma_{l}\right]
$$

where $a$ is a non-zero element of $\overline{\mathbf{Q}(q)}$. Hence we have

$$
M \ni v\left[\gamma_{l}\right]
$$

Therefore, if $\gamma_{l}$ is a simple loop around $\mathscr{D}_{0}$, the proof is completed.

Now suppose that $\gamma_{l}$ is a simple loop around $\mathscr{D}_{\infty}$. Let $\gamma_{l}^{\prime}$ be a simple loop around $\mathscr{D}_{\infty}$ as the one given at the beginning of $\S 9.6$ associated with a data $(\mathscr{A}$, $\left.c, b^{\prime}, \Delta\right)$. We can assume that the base point $b^{\prime}$ of the loop $r_{l}^{\prime}$ satisfies the following :

$$
b^{\prime} \in \mathcal{U}_{N}^{*} \subset \mathcal{U}_{N}^{\tilde{N}}
$$

By Proposition 9.1.1, there is a path $\alpha: I \rightarrow \mathcal{U}$ from $b$ to $b^{\prime}$ such that

$$
\left[\gamma_{l}\right]=[\alpha]^{-1}\left[\gamma_{l}^{\prime}\right][\alpha] \text { in } \pi_{1}(u, b)
$$

By Proposition 9.6.1 (1), we can write $\operatorname{Cr}(u)=\left\{p_{1}(u), \ldots, p_{N}(u)\right\}$ for $u \in$ $\Delta \backslash\{c\}$, where $p_{1}(u), \ldots, p_{N}(u)$ are holomorphic functions on $\Delta \backslash\{c\}$. By Proposition 9.6.1 (2), there is one and only one function among them, say $p_{N}(u)$, which has a pole at $u=c$. Let $\left[\xi_{N}^{\infty}\right] \in\left[\mathscr{P}_{b^{\prime}}\left(a_{b^{\prime}}^{\infty}, p_{N}\left(b^{\prime}\right)\right)\right]$ be the unique homotopy class of paths characterized by the property of being represented by a path $\xi_{N}^{\infty}$ such that

$$
\xi_{N}^{\infty}(I) \subset D_{N}^{\infty} \cap K_{b^{\prime}}
$$

where $D_{N}^{\infty}:=\left\{z \in \mathbb{C} \cup\{\infty\} ;|z| \geq\left|p_{N}\left(b^{\prime}\right)\right|\right\}$. This homotopy class is the one appeared in Proposition 9.7.2. Hence we can write $v\left[\gamma_{l}^{\prime}\right]$ in the form

$$
\begin{aligned}
v\left[\gamma_{l}^{\prime}\right]= & \Psi_{b^{\prime}}^{0}(e \otimes 1)+\Psi_{b^{\prime}}^{\infty}(w \otimes 1) \\
& +\Psi_{b^{\prime}}^{\infty}\left(\bar{\sigma}\left[\xi_{N}^{\infty}\right] \otimes\left( \pm q^{-d+1}+a_{-d+2} q^{-d+2}+\cdots+a_{-1} q^{-1}\right)\right) \\
\in & H_{n}\left(F_{b^{\prime}}, \partial_{0} F_{b^{\prime}}\right)
\end{aligned}
$$

by some $e \in H_{n-1}\left(X_{b^{\prime}}^{0}\right)$, some $w \in H_{n-1}\left(X_{b^{\prime}}^{\infty}\right)$ and some integers $a_{-1}, \ldots, a_{-d+2}$. 
Consider the element

$$
\tilde{v}\left[\gamma_{l}^{\prime}\right]:=(1-q) v\left[\gamma_{l}^{\prime}\right] \in H_{n}\left(F_{b^{\prime}}\right)
$$

Here we have used (6.16). By Lemma 6.1, we can write $(1-q) \Psi_{b^{\prime}}^{0}(e \otimes 1) \in$ $H_{n}\left(F_{b^{\prime}}\right)$ as $\Psi_{b^{\prime}}^{0}\left(e_{1} \otimes q+e_{0} \otimes 1\right)$ by some $e_{1}, e_{0} \in H_{n-1}\left(X_{b^{\prime}}^{\infty}\right)$. Putting this into (10.5), we see that $\tilde{v}\left[\gamma_{l}^{\prime}\right]$ is written in the form

$(10.6) \Psi_{b^{\prime}}^{\infty}\left(\alpha_{1} \otimes q+\alpha_{0} \otimes 1+\alpha_{-1} \otimes q^{-1}+\cdots+\alpha_{-d+2} \otimes q^{-d+2} \pm \bar{\sigma}\left[\xi_{N}^{\infty}\right] \otimes q^{-d+1}\right)$ where $\alpha_{1}, \ldots, \alpha_{-d+2}$ are certain elements of $H_{n-1}\left(X_{b^{\prime}}^{\infty}\right)$.

Let $\lambda_{N}^{0}$ be the path from $a_{b^{\prime}}^{0}=r$ to $p_{N}\left(b^{\prime}\right)$ given by

$$
\lambda_{N}^{0}(t):=(1-t) r+t \cdot p_{N}\left(b^{\prime}\right)
$$

By (10.2) and Lemma 9.5.1, $\lambda_{N}^{0}$ is an element of $\mathscr{P}_{b^{\prime}}\left(a_{b^{\prime}}^{0}, p_{N}\left(b^{\prime}\right)\right)$. By (10.2) and Proposition 9.5.1, there is a simple loop $\beta^{\prime}$ around $\mathscr{D}_{0}$ with the base point $b^{\prime}$ such that

$$
v^{\sim}\left[\beta^{\prime}\right] \equiv \bar{\theta}\left(\left[\lambda_{N}^{0}\right]\right)\langle 0\rangle=-\Psi_{b^{\prime}}^{0}\left(\bar{\sigma}\left[\lambda_{N}^{0}\right] \otimes 1\right) \text { in } H_{n}\left(F_{b^{\prime}}, \partial_{0} F_{b^{\prime}}\right)
$$

Here we have used Proposition 7.2. (Note that $\left[\lambda_{N}^{0}\right]$ is represented by a path contained in $K_{b^{\prime}}$ by Lemma 9.5.1.) We shall prove that

$$
\left[\beta^{\prime}\right]_{*}\left(\tilde{v}\left[\gamma_{l}^{\prime}\right]\right) \neq \tilde{v}\left[\gamma_{l}^{\prime}\right]
$$

Note that since $\tilde{v}\left[\gamma_{l}^{\prime}\right] \in H_{n}\left(F_{b^{\prime}}\right) \subset H_{n}\left(F_{b^{\prime}}, \partial_{\infty} F_{b^{\prime}}\right)$, we can apply Theorem 9.2.1 to the calculation of $\left[\beta^{\prime}\right]_{*}\left(\tilde{v}\left[\gamma_{i}^{\prime}\right]\right)$. By Theorem 9.2.1, in order to prove (10.9), it is enough to show that $\left(\tilde{v}\left[\gamma_{l}^{\prime}\right], v^{\sim}\left[\beta^{\prime}\right]\right)_{0}$ is not zero. By $(10.8)$, the Laurent polynomial $\left(\tilde{v}\left[\gamma_{l}^{\prime}\right], v^{\sim}\left[\beta^{\prime}\right]\right)_{0}$ is congruent with $\left(\tilde{v}\left[\gamma_{l}^{\prime}\right], \bar{\theta}\left(\left[\lambda_{N}^{0}\right]\right)\langle 0\rangle\right)_{0}$ modulo $U\left(\mathbb{Z}\left[q, q^{-1}\right]\right)$. Using the descriptions $(10.6)$ of $\tilde{v}\left[\gamma_{l}^{\prime}\right]$ and $(10.8)$ of $\bar{\theta}\left(\left[\lambda_{N}^{0}\right]\right)\langle 0\rangle$, and applying the formula (8.2), we see that the coefficient of $q^{-d+1}$ in the Laurent polynomial $\left(\tilde{v}\left[r_{l}^{\prime}\right], \bar{\theta}\left(\left[\lambda_{N}^{0}\right]\right)\langle 0\rangle\right)_{0}$ is the integer

$$
\pm\left\langle\Psi_{b^{\prime}}^{\infty}\left(\bar{\sigma}\left[\xi_{N}^{\infty}\right] \otimes 1\right), \Psi_{b^{\prime}}^{0}\left(\bar{\sigma}\left[\lambda_{N}^{0}\right] \otimes \dot{1}\right)\right\rangle= \pm\left(\bar{\theta}\left(\left[\xi_{N}^{\infty}\right]\right)\langle 0\rangle, \bar{\theta}\left(\left[\lambda_{N}^{0}\right]\right)\langle 0\rangle\right)_{0}
$$


By (10.4) and (10.7), the paths $\xi_{N}^{\infty}$ and $\lambda_{N}^{0}$ have a common ending point $p_{N}\left(b^{\prime}\right)$ as their only intersection point. Hence Lemma 8.2 implies that the integer (10.10) is \pm 1 . Thus (10.9) is proved.

Now we put $\beta:=\alpha^{-1} \beta^{\prime} \alpha$, which is a simple loop around $\mathscr{D}_{0}$ with the base point $b$. We also set

$$
\tilde{v}\left[\gamma_{l}\right]:=(1-q) v\left[\gamma_{l}\right] \in H_{n}\left(F_{b}\right) \cap M
$$

(Recall the relation (10.1).) From (10.3), we have $\tilde{v}\left[\gamma_{l}\right] \equiv[\alpha]^{-1}\left(\tilde{v}\left[\gamma_{l}^{\prime}\right]\right)$ by Corollary 9.7.1. Therefore (10.9) implies that

$$
[\beta]_{*}\left(\tilde{v}\left[\gamma_{l}\right]\right) \neq \tilde{v}\left[\gamma_{l}\right] .
$$

This implies $v[\beta] \in M$.

\section{References}

[1] Atiyah, M.F., Representations of braid groups, (notes by S.K. Donaldson), Geometry of Low-Dimensional Manifolds: 2, (S.K. Donaldson and C.B. Thomas, eds.), London Math. Soc. Lecture Note Ser., 151 Cambridge Univ. Press. (1990), 115-122.

[2] Deligne, P., Théorie de Hodge, II, Publ. Math. IHES, 40(1971), 5-57.

[3] - Le groupe fondamental du complément d'une courbe plane n'ayant que des points doubles ordinaires est abélien (d'aprés W. Fulton), Lecture Notes in Math., 842, Springer-Verlag, Berlin, 1981, 1-10.

[4] Fulton, W., On the fundamental group of the complement of a node curve, Ann. Math., 111 (1980), 407-409.

[5] Fulton, W. and Lazarsfeld, R., Connectivity and its applications in algebraic geometry, Lecture Notes in Math., 862, Springer-Verlag, Berlin, 1981, 26-92.

[6] Givental', A.B., Twisted Picard-Lefschetz formulas, Funct. Analy. Appli., 22 (1988), 10-18.

[7] Hamm, H.A. and Lê, D.T., Un théorème de Zarıski du type de Lefschetz, Ann. Sci. Ecole Norm. Sup., 6(1973), 317-366.

[8] Lamotke, K., The topology of complex projective varieties after S. Lefschetz, Topology, 20 (1981), 15-51.

[9] Lefschetz, S., L'Analysis Situs et la Géométrie Algébrique, Gauthier-Villars, Paris, 1924.

[10] Libgober, A., Alexander invariants of plane algebraic curves, Singularities, Part 2 (Arcata, 1981), Proc. Symp. Pure Math., 40 (1983), 135-143.

[11] - Homotopy groups of the complements to singular hypersurfaces, II, Ann. Math., 139 (1994), 117-144.

[12] Milnor, J., Morse Theory, Ann. of Math. Stud., 51, Princeton Univ. Press, 1963.

[13] Pham, F., Formules de Picard-Lefschetz généralisées et ramification des intégrales, Bull. Soc. Math. France, 93 (1965), 333-367.

[14] Zariski, O., A theorem on the Poincaré group of an algebraic hypersurface, Ann. Math., 38 (1937), 131-141. 
